\title{
WALL MODELED LARGE EDDY SIMULATION OF FLOW OVER A WALL MOUNTED HUMP
}

\author{
Deepu Dilip
}

Thesis submitted to the faculty of the Virginia Polytechnic Institute and State University in partial fulfillment of the requirements for the degree of

\author{
Master of Science \\ In \\ Mechanical Engineering \\ Danesh K. Tafti, Chair \\ Clinton L. Dancey \\ Mark R. Paul
}

April 21, 2014

Blacksburg, VA

Keywords: Computational Fluid Dynamics, Wall resolved Large Eddy Simulation, Wall Modeled Large Eddy Simulation, Two-layer Wall Model, Wall mounted hump

Deepu Dilip @ 2014 


\title{
Wall Modeled Large Eddy Simulation of Flow over a Wall Mounted Hump
}

Deepu Dilip

\begin{abstract}
Large Eddy Simulation (LES) is a relatively more accurate and reliable alternative to solution of Reynolds Averaged Navier Stokes (RANS) equations in simulating complex turbulent flows at a lesser computational cost than a direct numerical simulation (DNS). However, LES of wall-bounded flows still requires a very high grid resolution in the inner wall layer making its widespread use difficult. Different attempts have been made in the past time to overcome this problem by modeling the near wall turbulence instead of resolving it. One such approach is a two - layer wall model that solves for a reduced one-dimensional equation in the inner wall layer, while solving for the filtered Navier-Stokes equations in the outer layer. The use of such a model allows for a coarser grid resolution than a wall resolved LES.
\end{abstract}

This work validates the performance of a two-layer wall model developed for an arbitrary body fitted non-orthogonal grid in the flow over a wall mounted hump at Reynolds number 9.36 $\times 10^{5}$. The wall modeled large eddy simulation (WMLES) relaxes the grid requirement compared to a wall resolved LES (WRLES) by allowing the first off-wall grid point to be placed at a $\mathrm{y}^{+}$of approximately 20-40. It is found that the WMLES results are general good agreement with WRLES and experiments. Surface pressure coefficient, skin friction, mean velocity profiles, and the reattachment location compare very well with experiment. The WMLES and WRLES exhibit some under prediction of the peak values in the turbulent quantities close to the reattachment location, with better agreement with the experiment in the separated region. In 
contrast, a simulation that did not employ the wall model on the grid used for WMLES failed to predict flow separation and showed large discrepancies with the experimental data. In addition to the relaxation of the grid requirement in the wall normal direction, it was also observed that the wall model allowed a reduction in the number of computational cells in the spanwise direction by half. However an LES calculation on a grid with reduced number of cells in spanwise direction turned unstable almost immediately, thereby highlighting the effectiveness of the wall model. Besides reducing the number of grid points in the spatial domain, the relaxed grid resolution for the WMLES also permitted the use of a larger time step. This resulted in an order of magnitude reduction in the total CPU time relative to WRLES. 
To my parents 


\section{Acknowledgements}

First of all, I wish to thank Dr. Danesh Tafti, for helping me step into the world of CFD and for all the guidance he gave throughout this work. Be it explaining the fundamentals or reviewing this thesis, there was never a delay from his side. I also thank my committee members Dr. Clinton Dancey and Dr. Mark Paul for reviewing my research and serving on my committee. Special thanks to the Advanced Research in Computing at Virginia Tech for providing the computational resources without which the study wouldn't have been possible

I am grateful to my lab-mates Nagi and Kamal for helping me understand the code used for this research during my initial days in the lab; and also to Ravi for all the innumerable technical discussions I've had with him. I would also like to thank the other present and former members of HPCFD lab (Amit, Husam, Long, Naresh, Sukhi, Surya) who besides helping out in times of difficulty made the lab a great place to work at. Life at Virginia Tech was eventful and I thank David, Ryan, Sakshi, Shreyas \& Vivek for pulling me through the tough times and the friendship that evolved with it

Above all, I am grateful to my parents, whose support have been my biggest fortune, and also to my sister and brother-in-law for being there through the good and bad times. 


\section{Table of Contents}

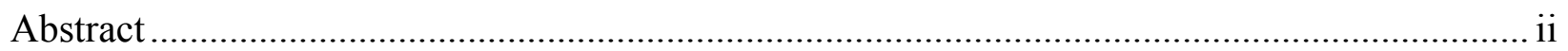

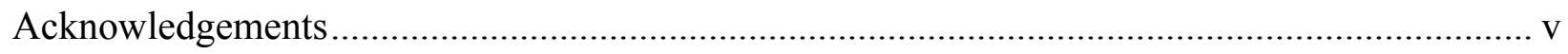

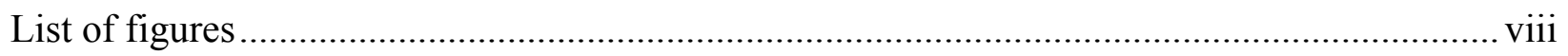

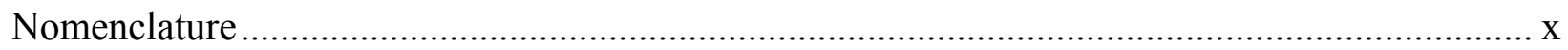

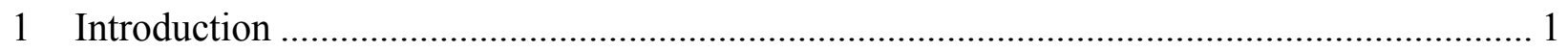

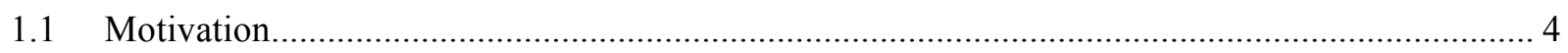

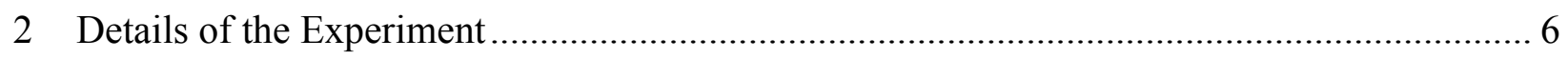

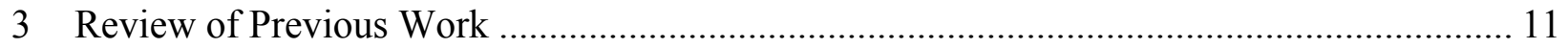

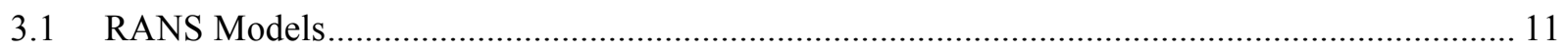

3.2 Direct Numerical Simulation (DNS) …………………………………………………. 15

3.3 LES, DES, DDES, Hybrid LES/RANS methods ………………………………………….... 16

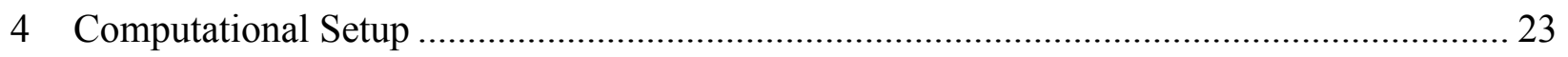

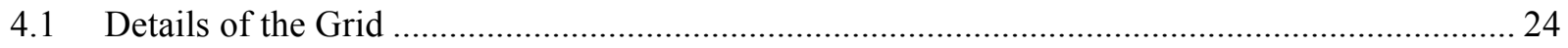

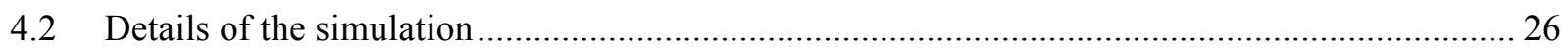

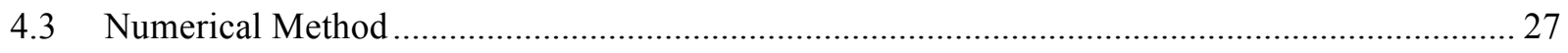

4.3.1 Governing Equations and sub-grid stress modeling …………………………………... 27

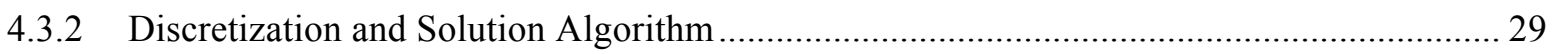

4.3.3 Parallel Implementation ...................................................................................... 32

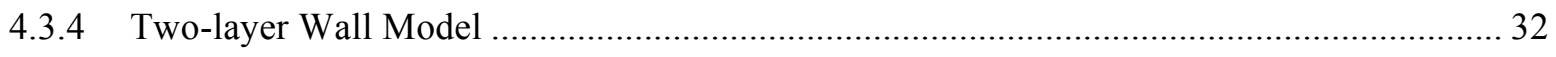

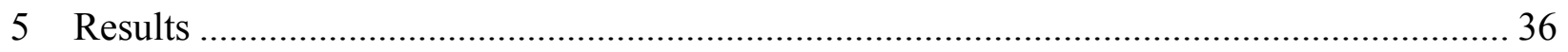

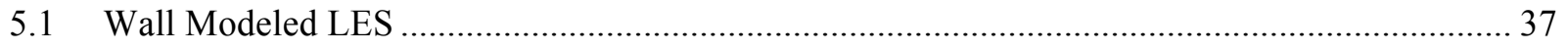

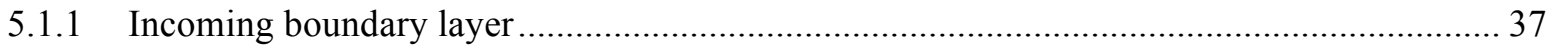




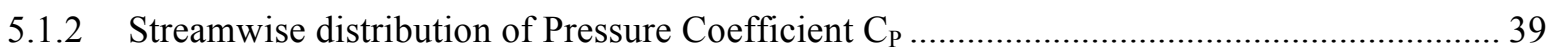

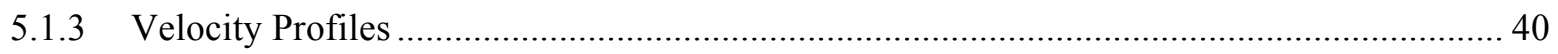

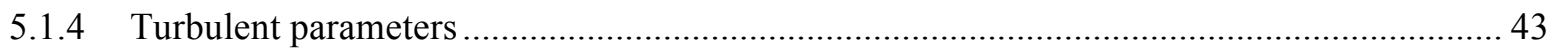

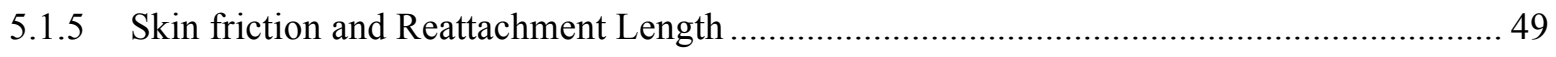

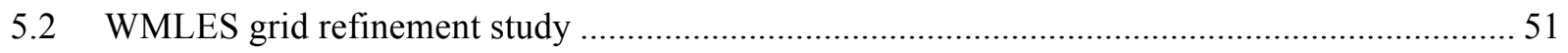

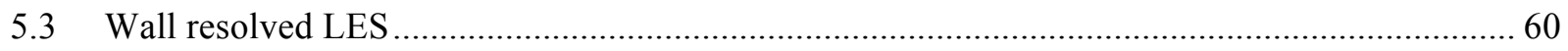

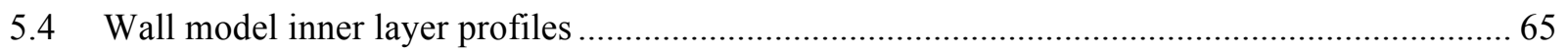

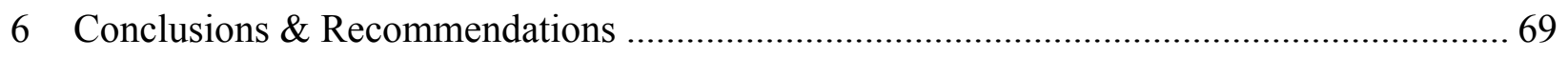

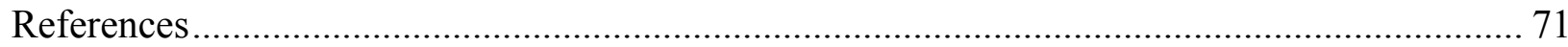

Appendix - Effects of accounting for end plates and grid refinement in wall normal direction.. 77

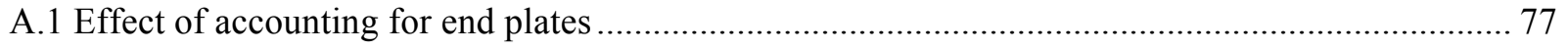

A.1.1 Modification to the computational domain .................................................................. 77

A.2 Effect of grid refinement in wall normal direction ............................................................... 81 


\section{List of Figures}

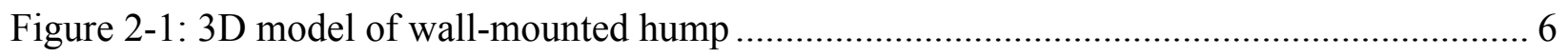

Figure 2-2 : Wind tunnel arrangement.................................................................................. 7

Figure 2-3 : Schematic of hump with important locations marked .............................................. 8

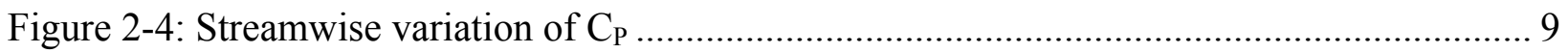

Figure 2-5: Profile of mean streamwise velocity ...................................................................... 9

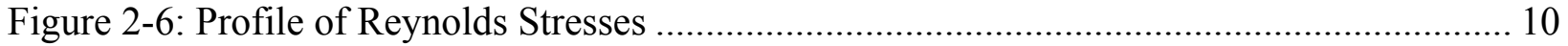

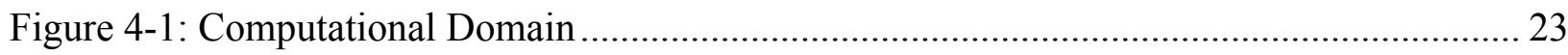

Figure 4-2: Resolution of grid along the domain...................................................................... 25

Figure 4-3: Computational grid; every $3^{\text {rd }}$ line shown in $\mathrm{x}$, every $2^{\text {nd }}$ line shown in y................. 25

Figure4-4: Virtual grid embedded between the LES grid............................................................. 34

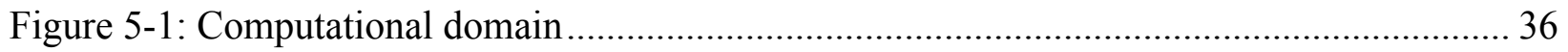

Figure 5-2: Left: WMLES, Right: WRLES ........................................................................ 37

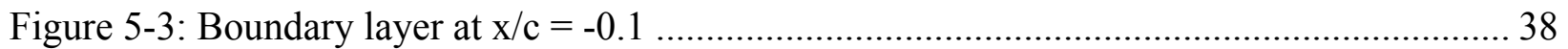

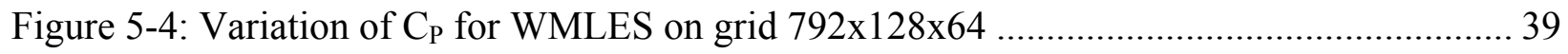

Figure 5-5: Mean velocity profiles from WMLES on grid 792x128x64 ……………………..... 41

Figure 5-6: Contour of mean u-velocity with streamlines from WMLES.................................... 42

Figure 5-7: Contour of mean u-velocity with streamlines when wall model not used .................. 43

Figure 5-8: Contour of turbulent TKE from WMLES .............................................................. 44

Figure 5-9: Contour of turbulent TKE without use of wall model ................................................ 44

Figure 5-10: Profile of $\overline{u^{\prime} u^{\prime}}$ from WMLES on grid 792x128x64 ............................................... 45

Figure 5-11: Profile of $\overline{v^{\prime} v^{\prime}}$ from WMLES on Grid 792x128x64 ……....................................... 46

Figure 5-12: Profile of $\overline{u^{\prime} v^{\prime}}$ from WMLES on grid 792x128x64 ............................................... 47

Figure 5-13: $\mathrm{C}_{\mathrm{f}}$ from WMLES on Grid 792x128x64............................................................ 50

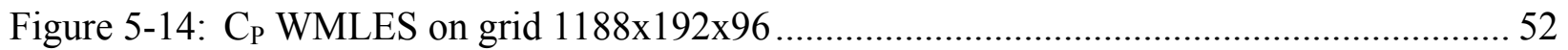

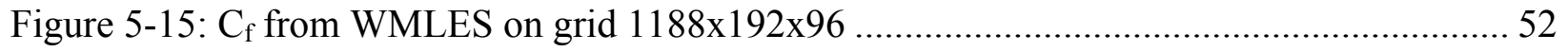

Figure 5-16: Profile of $\overline{u^{\prime} u^{\prime}}$ from WMLES on grid 1188x192x96 …........................................... 53

Figure 5-17: Profile of $\overline{v^{\prime} v^{\prime}}$ from WMLES on grid 1188x192x96 …………………….............. 54

Figure 5-18: Profile of $\overline{u^{\prime} v^{\prime}}$ from WMLES on grid 1188x192x96........................................... 54

Figure 5-19: Profile of mean streamwise velocity from WMLES on grid 1188x192x96 ............ 55

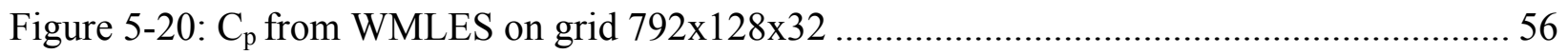




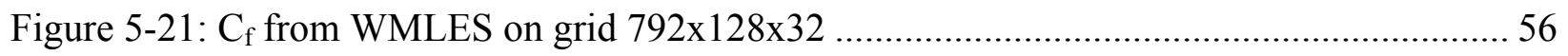

Figure 5-22: Profile of $u$ from WMLES on 792x128x32 …….............................................. 57

Figure 5-23: Profile of $\overline{u^{\prime} u^{\prime}}$ from WMLES on 792x128x32 …….............................................. 58

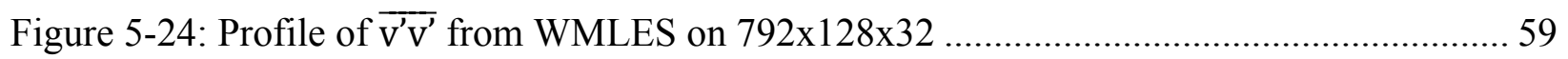

Figure 5-25: Profile of $\overline{u^{\prime} v^{\prime}}$ from WMLES on 792x128x32 ……………………...................... 59

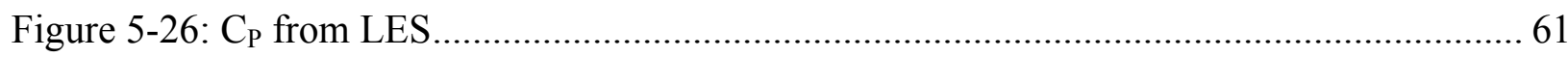

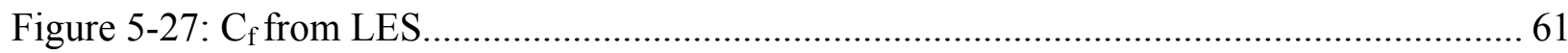

Figure 5-28: Profile of mean streamwise velocity from LES ......................................................... 62

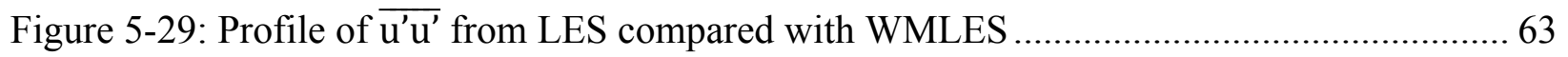

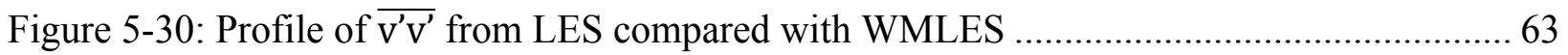

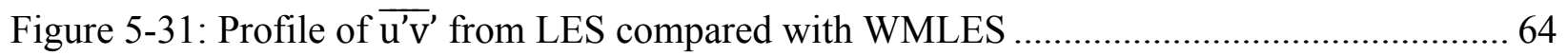

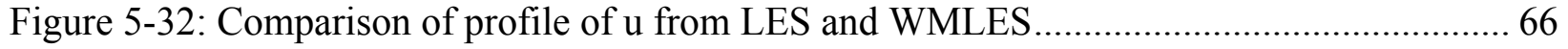

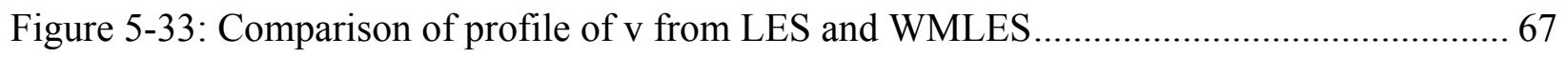

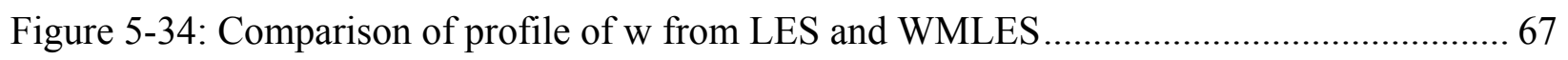

Figure A-1: Computational domain with modified top wall. ........................................................ 78

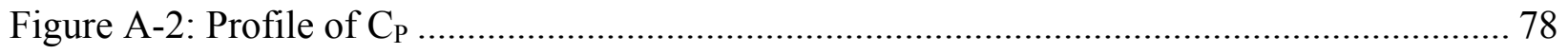

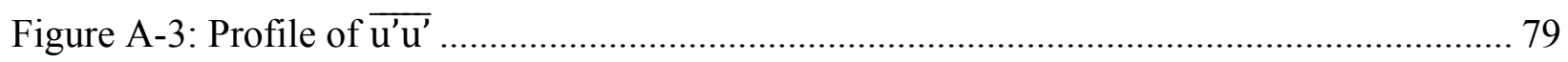

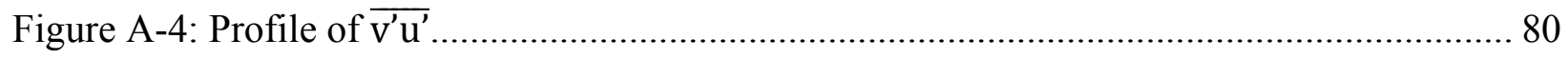

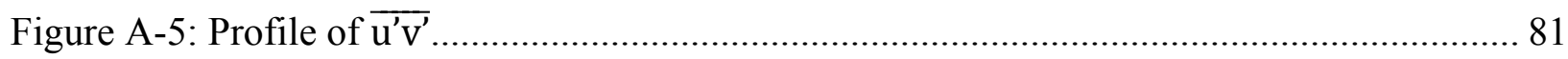

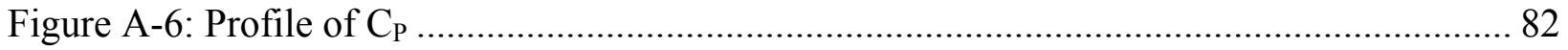

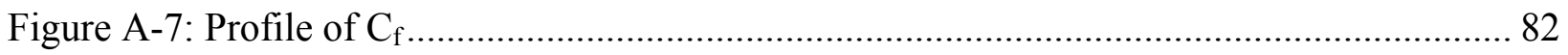

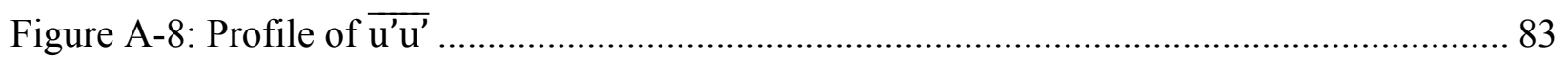

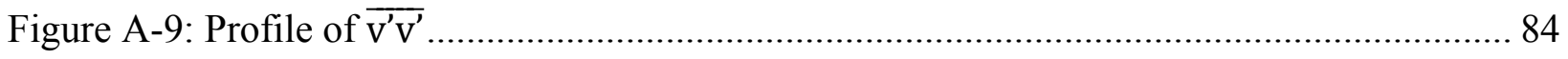

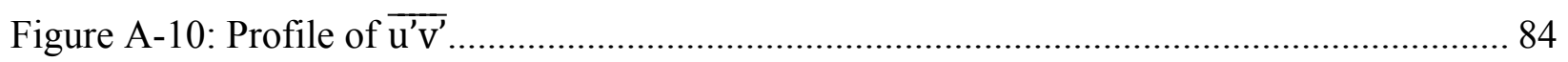

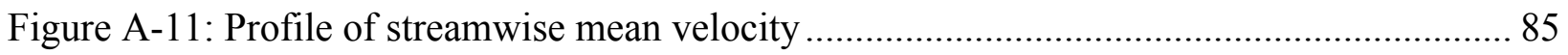




\section{List of Tables}

Table 3-1 Summary of previous work performed using RANS models ................... 12

Table 3-2 Summary of previous work performed using other turbulence models............ 16 


\section{Nomenclature}

\begin{tabular}{ll}
$c$ & Hump chord length \\
$\mathrm{C}_{\mathrm{P}}$ & Surface pressure coefficient \\
$p$ & Non dimensional static pressure \\
$\mathrm{Re}$ & Reynolds number \\
$t$ & Time \\
$\mathrm{U}_{\mathrm{t}}$ & Tangential velocity \\
$\sqrt{g}$ & Jacobian of transformation \\
$\sqrt{g} \mathrm{U}$ & Contravariant flux vector \\
$\mathrm{L}^{*}$ & Non-dimensional length \\
$\mathrm{U}^{*}$ & Non-dimensional velocity \\
$\xi, \eta, \zeta$ & Jacobians in the $x, y$ and $z$ directions \\
$v$ & Kinematic viscosity \\
$S_{i j}$ & Strain rate tensor \\
$C_{s}^{2}$ & Smagorinsky constant \\
$\kappa$ & von Karman constant \\
$M$ & Mach Number of the Flow \\
$\mathrm{d}$ & Normal distance from wall \\
$u_{\tau}$ & Friction velocity \\
$\tau_{w}$ & Wall shear stress \\
$R_{t}$ & Turbulent Reynolds Number \\
$\vec{t}$ & unit tangential vector \\
$\rho$ & Density \\
$\delta$ & Thickness of boundary layer \\
$U_{\infty}$ & Free Stream Velocity \\
$y^{+}$ & Non dimensional wall coordinate \\
$t$ & Dimensionless Wall Distance \\
$t^{*}$ & Reference Dimensional Time Scale \\
$x$ & X coordinate \\
$y$ & Y Coordinate \\
$z$ & Z Coordinate \\
$s u p e r s c r i p t s$ & \\
$(\bar{~})$ & Filtered quantity \\
, & Fluctuating quantity \\
& \\
\hline &
\end{tabular}




\section{Introduction}

Large eddy simulation (LES) offers a compromise between solution to Reynolds Averaged Navier Stokes equations (RANS), that are relatively less accurate but computationally less expensive, and direct numerical simulations (DNS), that are more accurate but extremely demanding in computational resources. As an intermediate technique, LES only resolves the large-scale unsteady energy-carrying geometry dependent anisotropic eddies, while modeling the effect of the smallest scales in the flow. This allows LES to achieve higher accuracy than RANS simulations without the extreme grid requirements of DNS. Although the early applications of LES were limited to simple flows such as plane channel flows, free shear flows, homogeneous turbulence etc., its use today have been extended to more complex engineering flows as computers have become more powerful.

Despite the advancements in computing power, the grid requirement for an accurate large eddy simulation at practical Reynolds numbers, which could be of the order of several hundred thousand to millions, is still too large for its widespread application. This is particularly true in the case of wall proximal flows, which demand very fine grids for the accurate resolution of near wall turbulence. In wall-proximal flows, the number of computational cells required to resolve the energy producing structures in the near-wall region scale as $R e^{1.8}$, whereas by contrast, the outer layer resolution scales as $R e^{0.4}[1]$. The extremely fine grid in the wall proximal inner layer increases the computational cost, not only due to increased number of grid points in the spatial domain but also due to the resulting small time step, which is limited by stability and accuracy 
requirements. Thus, the application of LES to high Reynolds number engineering flows can only be realized by reducing the computational cost.

A solution to circumvent this problem is to model the near wall turbulence instead of resolving the very small scales in the near wall region. This can lead to significant reduction in the computational cost, not just through a reduction in the number of computational cells in the spatial domain, but also through the subsequent increase in the permissible time step. A number of different approaches for modeling the near wall layer already exist, viz., the use of logarithmic law of the wall based functions, solving a separate set of equations in the near-wall region, simulating this region in a Reynolds-averaged sense etc. For instance, the method of Deardorff [2] and Schumann [3] models the effect of near wall layer through approximate wall boundary conditions, wherein information from the outer flow is used to determine local wall stress, which is in turn fed back to the outer LES in the form of momentum flux at wall due to normal diffusion. However, the method requires a value of mean wall shear stress a priori besides having the plane averaged velocity at first off-wall grid point to explicitly satisfy the logarithmic law, thereby limiting its applications to simple equilibrium flows.

Hybrid RANS-LES is another approach that has become popular in recent years, where RANS equations are solved near the wall which transition to the LES filtered Navier-Stokes far from the wall [4-7]. The Detached Eddy Simulation proposed by Spalart et al. [4] is an example, where the one-equation SA model in the RANS region, with a characteristic turbulent length scale is used to switch between the RANS and LES regions. Several other hybrid methods were proposed later, which solved for other two-equation turbulence models instead of the SA model in the RANS region with the characteristic turbulent length scales obtained from the model itself 
[7]. The hybrid RANS-LES models have been shown capable in simulating in a number of complex flows [8-11]. However, their application still requires the first grid point satisfy $\mathrm{y}^{+}<1$ in the wall normal direction, though it relieves the resolution requirement in wall parallel directions.

In a zonal model or two-layer model, a different set of equations is solved on a virtual grid in the inner wall layer. Here, it is assumed that the outer and near wall regions are weakly coupled. In this approach, a virtual grid is embedded between the wall and the first off-wall grid point, refined only in the wall normal direction. In the outer LES grid, the filtered Navier-Stokes equations are solved, while in the inner layer eqn. (1.1) is solved.

$$
\frac{\partial \bar{u}_{i}}{\partial t}+\frac{\partial}{\partial x_{i}}\left(\bar{u}_{n} \bar{u}_{i}\right)=-\frac{1}{\rho} \frac{\partial \bar{p}}{\partial x_{i}}+\frac{\partial}{\partial x_{n}}\left[\left(v+v_{t}\right) \frac{\partial \bar{u}_{i}}{\partial x_{n}}\right]
$$

where $n$ is the wall normal direction and $i$ takes values 1,2 or 1,3 or 2,3 based on the wall orientation. The wall normal velocity $u_{n}$ is computed using mass conservation in the inner layer. Eqn. (1.1) is solved using the no-slip boundary condition at the wall, and the velocity at the first grid point off the wall which is calculated from the outer-flow LES. Integration of eqn. (1.1) in the inner layer yields the streamwise and spanwise components of the wall-stress and this is used as boundary condition for the outer-flow LES calculation. The inner layer calculations take a very small percentage of the total cost of the whole calculation. Besides, this method does not require the solution of pressure Poisson equation in the inner layer since the pressure field just outside the inner layer is imposed on the inner layer. These aspects make the procedure very inexpensive in comparison to the wall layer resolved LES. The two-layer model has been found to be effective in a variety of problems in the past, for instance, flow over a backward facing step 
[12], flow past an asymmetric trailing edge [13], three dimensional flow around a hill shaped obstruction [14] etc.

The work of Patil and Tafti [15] further developed on the two-layer model extending its application to complex geometries where computational grids are no longer orthogonal. As per their model, the equations are formulated in local wall coordinates $(n, t)$ within the inner layer. This enables its application in case of a general body-fitted non-orthogonal grid in which the normal and tangential velocities are typically not aligned with the $\mathrm{x}, \mathrm{y}$ and $\mathrm{z}$ coordinates as in the case of Cartesian geometries. This modified two-layer model has been successfully applied in a

number of cases such as turbulent channel flow up to $\mathrm{Re}=$ of $2 \times 10^{6}$ [15], a backward facing step at $\operatorname{Re}=40,000$ [15], swirl flows in a swirl combustor at $\operatorname{Re}=20,000$ [15] and can combustor at $\operatorname{Re}=80,000$ [16] and flow in a ribbed duct passage for Re up to 60,000 [17].

\subsection{Motivation}

The objective of this work is to validate the two-layer wall model of Patil and Tafti [15] in the NASA challenge problem, two-dimensional flow over a wall-mounted hump [18-20]. This was one of the test cases considered in the workshop on CFD validation of synthetic jets and turbulence separation control conducted by NASA in 2004. The validation studies done earlier used simple geometries at high Reynolds numbers, as in the case of the turbulent channel flow, or a more complicated geometry at lower Reynolds numbers, as in the case of backward facing step, ribbed ducts etc. Hence there are three primary reasons behind the choice of this problem for this study. Firstly, this is a case of boundary layer separation and reattachment, which although widely seen in a number of engineering design applications, (e.g, design of airfoils), remains a challenge for numerical simulations to predict accurately. Secondly, the Reynolds number of the flow is approximately 1 million, which increases the complexity of the problem in 
a computational perspective. And finally, the case being an experiment performed with an objective to provide the CFD community with a complete data set for validation purposes, a large amount of experimental data is available to compare the results obtained from a simulation.

This thesis is organized as flows. Chapter 2 describes the detailed configuration of the experiment and the data available from the same for comparison. Chapter 3 reviews the previous studies on the same problem using difference turbulence modeling techniques such as solution to Reynolds Averaged Navier Stokes equations (RANS), Large Eddy Simulaion (LES), Detached Eddy Simulation (DES) etc. It shall be seen from chapter 3 that LES enable better prediction of the experimental parameters than RANS models, though at an increased computational cost. Chapter 4 details the computational setup and the numerical methodology employed for all the studies in this work. Chapter 5 details the results and observations from the current study. Chapter 6 summarizes the conclusions from this study. 


\section{Details of the Experiment}

A detailed description of the experiment can be found in Greenblatt et al. [18, 19] and Naughton et al. [20] The experimental setup consists of a Glauert-Goldschmied type body, which is a contoured, nominally two-dimensional hill with a convex fore-body and a concave afterbody. Figure 2-1 shows a 3D model of the wall-mounted hump.

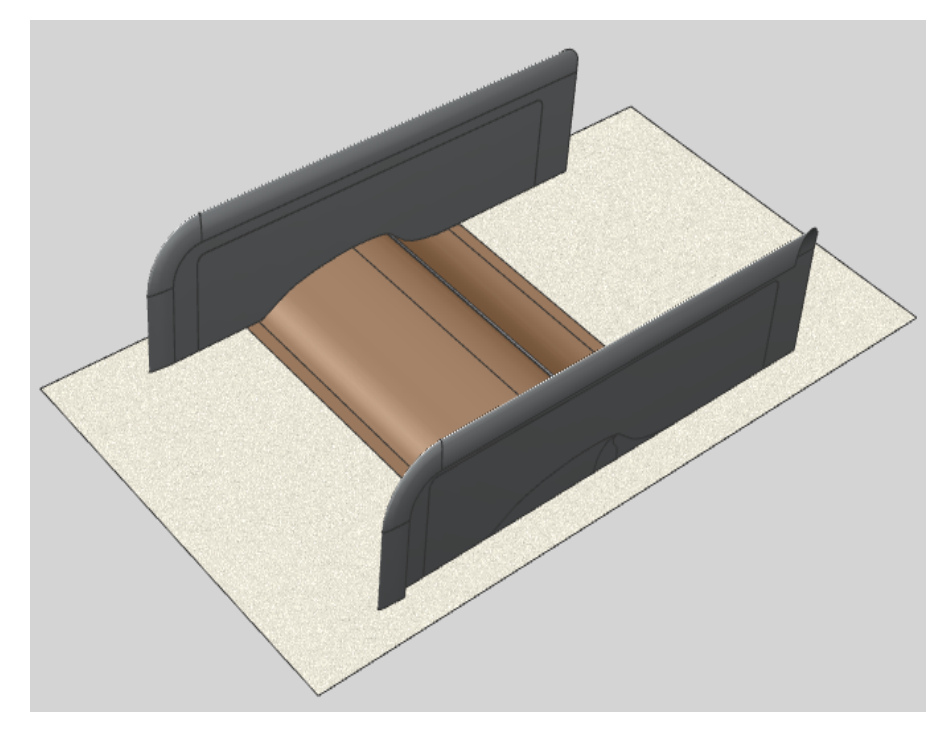

Figure 2-1: 3D model of wall-mounted hump

The experimental model is mounted between two glass endplate frames, and both leading edge and trailing edge are faired smoothly with a wind tunnel splitter plate. The length of the hump on the splitter plate, which is equal to 16.536 inches, is defined as the characteristic reference "chord" length of the model. The tunnel dimensions at the test section are 28 inches wide by 20 inches high. Since the hump model is mounted on the splitter plate that is 0.5 inches thick, the test section has a nominal height of 15.032 inches, which is the distance from the 
splitter plate to the top wall. The model is 23 inches wide between the endplates, and 2.116 inches high at its maximum thickness point. The splitter plate extends 76.188 inches upstream of the leading edge of the hump. Figure 2-2 shows the wind tunnel set up

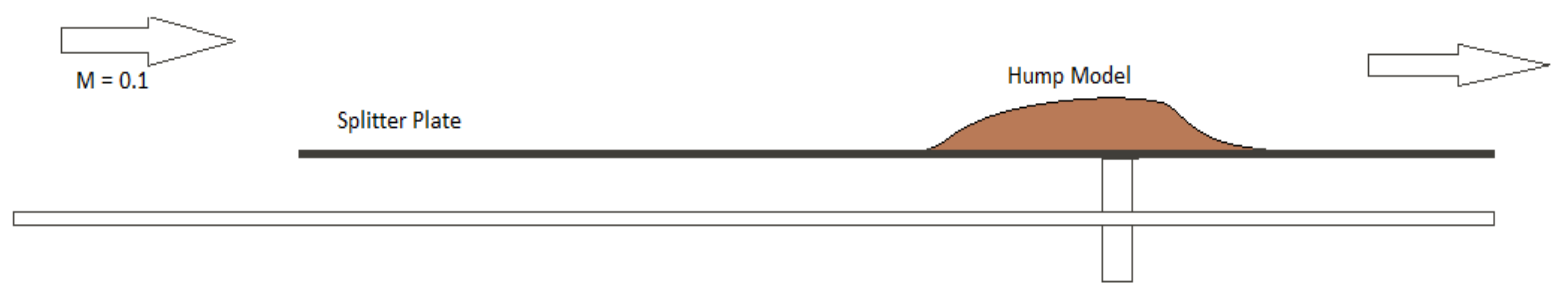

Figure 2-2 : Wind tunnel arrangement

The experiment is a case of flow separation followed by reattachment, which is similar to the work of Siefert \& Pack [21] who studied the active flow separation control at high Reynolds number. The geometry is also similar to that of Siefert \& Pack [21], and physically larger by a factor of 2 . The flow separates at approximately $\mathrm{x} / \mathrm{c}=0.67$ and reattaches at around $\mathrm{x} / \mathrm{c}=1.11$, where $\mathrm{x} / \mathrm{c}=0$ is at the base of the hump.

The experiment was performed under conditions of $\mathrm{M}=0.1$ and $\mathrm{Re}=9.36 \times 10^{5}$ based on the approach velocity and chord. The data acquired for the case included surface static and dynamic pressures, and two-dimensional and stereo (three-dimensional) PIV in the separated and reattachment regions. An independent check of the PIV flow field was done with hot wire and pitot tube data. An oil-film technique was used to determine the reattachment location. The inflow and upper wall boundary layers were also documented. 
The boundary layer approaching the hump was observed to be fully turbulent with a thickness of approximately 1- 2 inches. The boundary layer is subjected to a favorable pressure gradient over the front convex portion of the body and separates over a relatively short concave section in the aft part of the body. As for boundary conditions for the simulation, the experiment provides upstream data associated with the boundary layer at the location $\mathrm{x} / \mathrm{c}=-2.16$ where $\mathrm{x} / \mathrm{c}=$ 0 is the beginning of the fore-body of the hump, and $\mathrm{c}$ is the chord of the hump. This includes streamwise mean velocity and turbulence intensity profile. For CFD validation purposes, experimental data is provided for streamwise surface pressure coefficients and skin friction coefficient for the length of the body, profile of mean velocity and Reynolds stresses at selected data points in the aft part of the body and location of flow reattachment following separation. The data for the above parameters are provided for a baseline case, where no flow control is performed, a case with steady suction of the boundary layer, and with unsteady oscillatory flow control.

The experimental data for the parameters mentioned above are available at the locations marked in the figure 2-3 below.

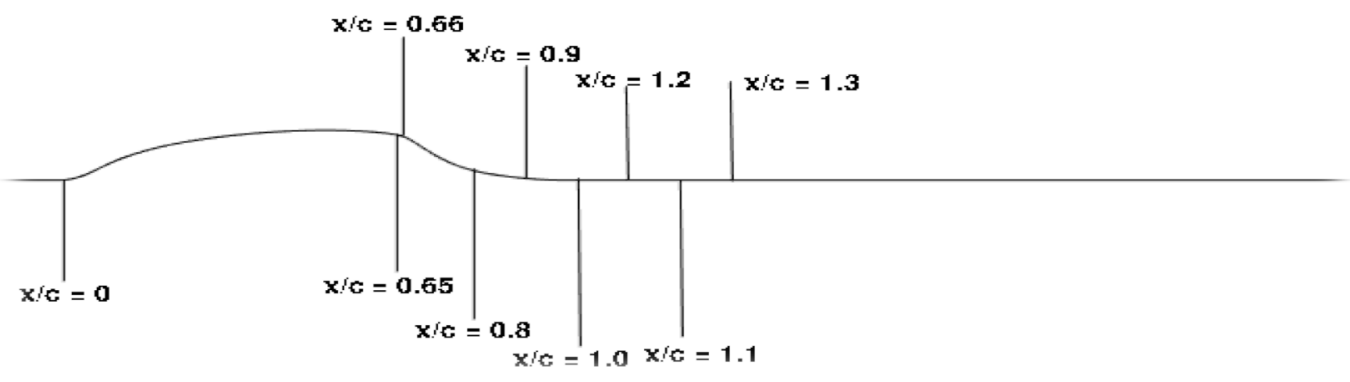

Figure 2-3 : Schematic of hump with important locations marked 


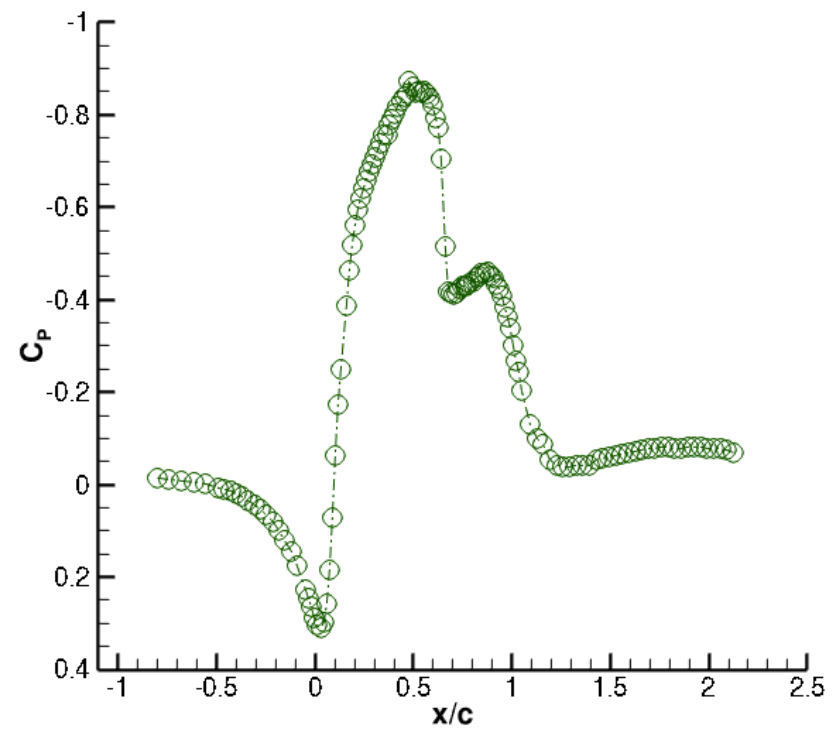

Figure 2-4: Streamwise variation of $C_{P}$

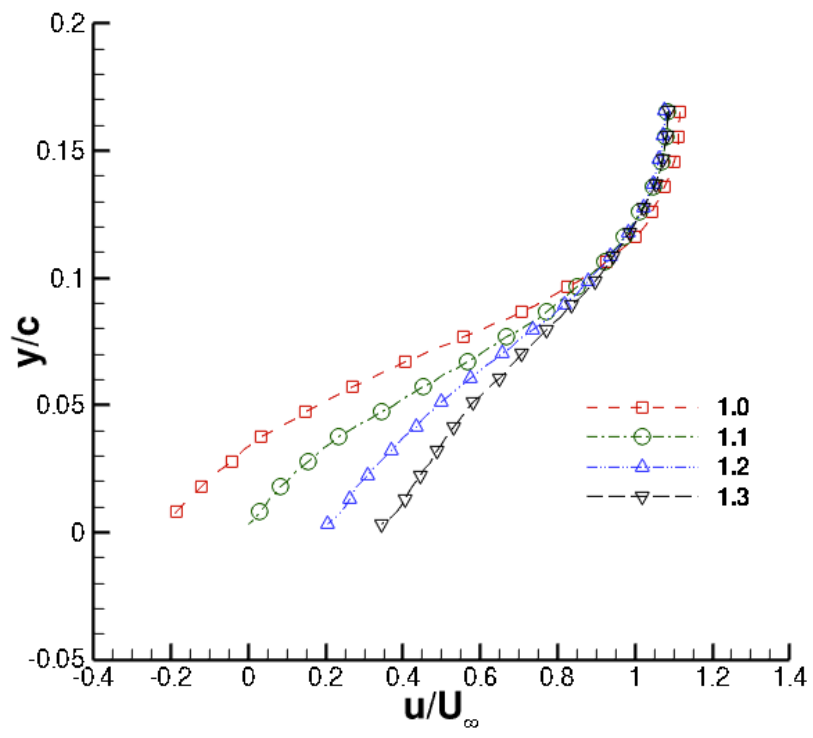

Figure 2-5: Profile of mean streamwise velocity

Figure 2-4 shows the plot of streamwise variation of the pressure coefficient. At the model leading edge, i.e., the base of the hump, the flow decelerates generating a partial stagnation point. The flow then accelerates rapidly for the first $15 \%$ of the hump, after which the acceleration decreases up to $\mathrm{x} / \mathrm{c}=0.5$, where the first suction peak is located. A pressure recovery then starts and continues until approximately $\mathrm{x} / \mathrm{c}=0.67$, where the flow separates. A large separation bubble is then formed at the lee side of the hump. The flow in the bubble accelerates initially, indicating spreading of the mixing layer. The separated mixing layer then starts interacting with the surface, turning towards it at approximately $\mathrm{x} / \mathrm{c}=0.9$, with the flow reattaching at $\mathrm{x} / \mathrm{c}=1.11$.

Figure 2-5 shows the profile of mean streamwise velocity in the vicinity of the reattachment point. The velocity in the region of the boundary layer close to the surface changes direction from location $\mathrm{x} / \mathrm{c}=1.0$ to $\mathrm{x} / \mathrm{c}=1.2$, indicating that reattachment occurs in between. The boundary layer further accelerates as shown by the higher velocity at location $\mathrm{x} / \mathrm{c}=1.3$ 

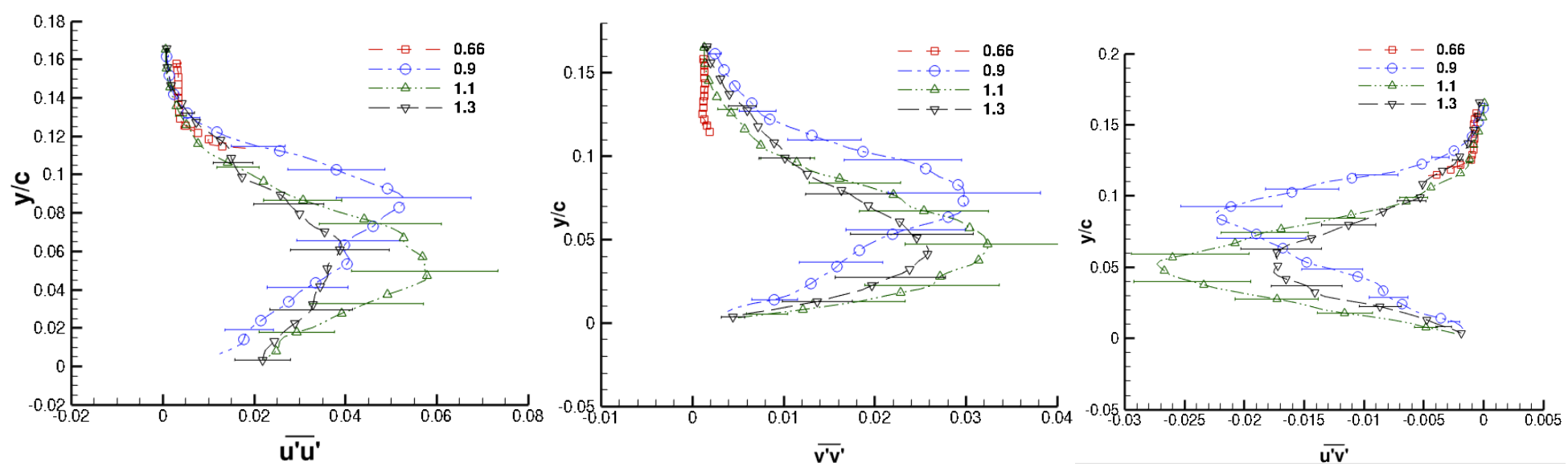

Figure 2-6: Profile of Reynolds Stresses

Figure 2-6 shows the profiles of Reynolds stresses at locations that are just upstream of separation point $(\mathrm{x} / \mathrm{c}=0.66)$, within the separation bubble $(\mathrm{x} / \mathrm{c}=0.9,1.1)$ as well as downstream of the reattachment point $(\mathrm{x} / \mathrm{c}=1.3)$. It is to be noted here that the experiment reported a maximum uncertainty of $14 \%$ for $u_{\mathrm{rms}}$ and $\mathrm{v}_{\mathrm{rms}}$ and $20 \%$ for $\overline{u^{\prime} v^{\prime}}$. Hence, it was suggested in the CFD validation workshop, that the profiles of turbulent parameters be used only for a qualitative comparison with the results from computation. To emphasize on this fact, the error bars calculated with the uncertainties mentioned above are also shown in the figures. The turbulence is low close to the separation point, as indicated by the magnitude of the normal and shear stresses at location $\mathrm{x} / \mathrm{c}=0.66$. Once within the separation bubble, the level of turbulence increases, as indicated by the profiles at location 0.9. Beyond this point, there is a strong adverse pressure gradient with a further increase in the turbulence levels, resulting in highest magnitudes of the turbulent stresses at $\mathrm{x} / \mathrm{c}=1.1$. The peak in the turbulent shear stress at $\mathrm{x} / \mathrm{c}=1.1$ indicates the flow approaching reattachment. The turbulence intensity decreases as the flow proceeds beyond reattachment. 


\section{Review of Previous Work}

The flow over a wall-mounted hump was one among the three test cases presented at the workshop on CFD validation and separation control conducted by NASA. Hence, a number of computational studies have been done till date. The majority of the previous works have employed RANS/URANS models, while few attempts using other methods such as Hybrid RANS-LES, LES, Implicit LES (ILES) and DNS methods have also been performed. This section summarizes the various approaches used in the past and the observations from them.

\subsection{RANS Models}

As mentioned earlier, the majority of the previous studies employed various RANS models such as Spalart-Allmaras model (SA), standard $k-\omega$ model, Shear Stress Transport model (SST), SST with reattachment modification, standard $\mathrm{k}-\varepsilon$, and several other variants of the $\mathrm{k}-\varepsilon$ and the $\mathrm{k}-\omega$ model such as $\mathrm{k}-\varepsilon$ LS (Launder \& Sharma [22]), $\mathrm{k}-\varepsilon$ AKN ( Abe, Kondoh \& Nagano [23]), AJL $-\varepsilon$ model which is a non-linear $\mathrm{k}-\mathrm{k}-\varepsilon$ model ( Abe, Jang \& Leschziner [24]) and AJL - $\omega$ model, which is a non-linear $\mathrm{k}-\omega$ model (Abe, Jang \& Leschziner [24]). This section briefly describes the observations from the use of the above models to predict the flow. Table (3.1) lists the different studies performed using various RANS models

\begin{tabular}{|c|c|c|}
\hline SL No. & Authors & RANS Models Used \\
\hline 1 & Krishnan et al. & SA, SST \\
\hline 2 & Balakumar & SST \\
\hline 3 & Cui et al. & SST \\
\hline 4 & Rumsey et al. & \\
\hline 5 & Hiller et al. & $\mathrm{k}-\omega$, SST, SST with reattachment modification, \\
\hline
\end{tabular}




\begin{tabular}{|c|c|c|}
\hline 6 & He et al. & k- $\varepsilon, \mathrm{k}-\omega$, SST, S-A \\
\hline 7 & Madugundi et al. & $\begin{array}{r}\text { SA, k- } \omega \text { (standard, SST), } \\
\text { k- } \varepsilon,(\text { Standard, RNG, realizable) }\end{array}$ \\
\hline 8 & Yeshala et al. & SST \\
\hline 9 & Yoshio et al. & $\begin{array}{r}\text { k- } \omega \text { SST, k- } \varepsilon \text { LS, k- } \varepsilon \text { AKN, non-linear k- } \varepsilon \text { (AJL- } \\
\varepsilon), \text { non-linear k-w (AJL }-\omega)\end{array}$ \\
\hline 10 & Gan et al. & SST \\
\hline 11 & Shakib et al. & SA \\
\hline
\end{tabular}

Table 3-1 Summary of previous work performed using RANS models

Among the different RANS models, Menter's Shear Stress Transport model was used in a number of studies. It was observed that in general, not accounting for the effect of end walls in the computational domain, as in the case of Balakumar [25], Cui et al. [26], Hiller et al. [27], Madugundi et al. [28] and Yoshio et al. [29], resulted in under prediction of the suction peak near the separation point in the $C_{P}$ profile. An exception to this was the work of Yeshala et al. [30], who made observations similar to others despite making modifications to the top wall in the computational domain. He et al. [31] accounted for the end plate blockage in their computational domain and had, however, observed accurate prediction of the suction peak due to end plate blockage in their work. In general, all the data reported for mean velocity profiles show differences with experiment. Balakumar [25] and Cui et al. [26] observed that the simulations tend to agree relatively better with the experiment in the recirculation region, particularly around $\mathrm{x} / \mathrm{c}=0.8$ and 0.9 . For locations downstream of $\mathrm{x} / \mathrm{c}=1.0$, larger difference between computational and experimental data were observed. The data for turbulent shear stress as reported by Balakumar [25] and Yeshala [30] show major under prediction of turbulent shear stress at all locations. For the reattachment location, Balakumar [25], Cui et al. [26] and Yoshio et. al [29] reported a value of $1.276,1.19$ and 1.18 respectively. 
Hiller et al. [27] also used an SST model with a reattachment modification proposed by Menter for cases of separated flow over curved surfaces. The profile of $C_{P}$ in this case agreed better with experiment in comparison to the standard SST model, with lesser under prediction of the suction peak near the separation point.

The studies of Krishnan et al. [32], Rumsey et al. [33], He et al. [31], Madugundi et al. [28] and Shakib et al. [34] employed the Spalart-Allmaras model. Krishnan et al. [32] and Rumsey et al. [33] observed that accounting for end plate blockage results in better prediction of the suction peak near the separation point in $\mathrm{C}_{\mathrm{P}}$. Madugundi et al. [28] observed greater discrepancy of computed mean velocity profiles with experiment using SA model than the SST model while the opposite was observed by Krishnan et al. [32]. He et al. [31] also observed some discrepancy with experiment in mean velocity profiles, with the computed profiles being closest to the experimental profiles in the recirculation region. As for the Reynolds stresses, Krishnan et al. [32] and He et al. [31] observed under predicted values for turbulent shear stresses with respect to the experiment for the locations reported. For the reattachment length, Rumsey et al. [33] obtained a value of 1.24, Shakib et al. [34] a value of 1.22 and Krishnan et al. [32] a value of 1.28

Hiller et al. [27] and Madugundi et al. [28] studied the standard k - $\omega$ model for this case. Hiller et al. [27] observed a trend for $C_{P}$ similar to the case of SST with reattachment modification mentioned earlier, with more under prediction in the separated region. Madugundi et al. [28] obtained a profile for $\mathrm{C}_{\mathrm{P}}$ that was similar to the one obtained from the SA model, and showed greater under prediction in comparison to the profile obtained from the SST model. In 
predicting the mean velocity profiles, Madugundi et al. [28] observed that the model performed better than the SA model but not as good as SST model.

He et al. [31] and Madugundi et al. [28] employed the standard $\mathrm{k}-\varepsilon$ model. Madugundi et al. [28] observed a $C_{P}$ profile very close to the ones obtained from the SA model and $k-\omega$ model up to the reattachment point, with continued under prediction of the suction peak near the separation point. It is interesting to note that He et al. [31] under predicted the second suction peak in the profile of $C_{P}$ using this model despite making modifications to the top wall of the computational domain, an observation not seen with the other models they employed. For mean velocity profiles and turbulent shear stresses, He et al [31] observed that the results were similar to those from SA model in trends and degree of agreement with experimental data. Madugundi et al. [28] however observed the model to be better than SA model in predicting these parameters, although the under prediction was greater than the SST and $\mathrm{k}-\omega$ models.

Several other works have used variants of the $\mathrm{k}-\varepsilon$ and $\mathrm{k}-\omega$ models. Yoshio et al. [29] used two linear $\mathrm{k}-\varepsilon$ models (LS model [22], AKN model [23]), a non-linear $\mathrm{k}-\mathrm{k}-\varepsilon$ model (AJL $-\varepsilon$ model [24]) and a non-linear $\mathrm{k}-\omega \operatorname{model}(\mathrm{AJL}-\omega$ model [24]) to study this problem. They obtained reattachment locations of $1.18,1.28,1.16,1.29$ and 1.22 respectively for the models mentioned above. Madugundi et al. [28] also used the $\mathrm{k}-\varepsilon$ Realizable model and $\mathrm{k}-\varepsilon$ RNG model for this problem. They obtained the same trend and level of agreement for $\mathrm{C}_{\mathrm{P}}$ as the SST model for both the models. For velocity profiles, both the models show under prediction, with $\mathrm{k}-\varepsilon$ Realizable agreeing closely with the results from SST, and $\mathrm{k}-\varepsilon$ RNG showing more under prediction. 
To summarize, in general, the prediction of various flow parameters such as distribution of coefficient of pressure, profile of mean velocity and Reynolds stresses, reattachment length etc. from the use of RANS models were not very accurate, with discrepancies being exhibited with the experimental data. The performance of one RANS model over the other was not consistent among different studies. A few points can however be inferred from the analysis of the works mentioned above. First, accounting for the end plate blockage, in general, results in better prediction of the second suction peak in the streamwise profile of $\mathrm{C}_{\mathrm{P}}$. Second, the velocity profiles tend to agree the most with the experiment in the recirculation region, in particular, around $\mathrm{x} / \mathrm{c}=0.8$ and $\mathrm{x} / \mathrm{c}=0.9$. Third, the Reynolds stresses are in general under predicted everywhere.

\subsection{Direct Numerical Simulation (DNS)}

Postl et al. [35] performed a Direct Numerical Simulation for the flow over the hump at the same Reynolds number as that of the experiment. The work solved for the velocity vorticity formulation of the Incompressible Navier-Stokes equation in a general orthogonal coordinate system using fourth order accurate compact differencing schemes for spatial discretization and fourth order accurate Runge-Kutta scheme for time integration. The computational grid consisted of 2049,321 and 160 points over a distance of $3.25,0.9$ and 0.071 units in the $\mathrm{x}, \mathrm{y}$ and $\mathrm{z}$ direction respectively. A laminar Blasius boundary layer profile was imposed as inflow boundary condition, which was tripped to turbulence near the inflow boundary by introducing a high amplitude 3D disturbance into the flow. However the boundary layer profile did not precisely match the turbulent profile provided by the experiment since the experimental location was far upstream beyond the computational domain. The observed streamwise pressure coefficient from their work showed under prediction of the suction peak near the separation point, caused due to the blockage effect of the endplates, whose effect was not accounted for in the computational domain. The reattachment 
length was over predicted by $20 \%$, with the reattachment observed at $\mathrm{x} / \mathrm{c}=1.19$. The streamwise and vertical velocity profiles showed good agreement with the experiment in the recirculation region, with slight discrepancies beyond reattachment. The predicted Reynolds stress components showed good agreement in general, with minor discrepancies at few locations.

\subsection{LES, DES, DDES, Hybrid LES/RANS methods}

Table (3.2) lists the different studies performed using various other methods for modeling turbulent flows such as Large Eddy Simulation (LES), Implicit Large Eddy Simulation (ILES) Detached Eddy Simulation (DES), Delayed Detached Eddy simulation (DDES) and Zonal Hybrid LES/RANS (HLR) methods. A short description of these methods shall be presented later.

\begin{tabular}{|c|c|c|}
\hline SL No. & Authors & Method used \\
\hline 1 & Saric et al. & LES, DES, DDES, HLR \\
\hline 2 & Lyons et al. & DES, Zonal DES, DDES \\
\hline 3 & You et al. & Implicit LES \\
\hline 4 & Morgan et al. & Compressible LES \\
\hline 5 & Frank et al. & Implicit LES \\
\hline 6 & Avdis et al. & Higher order LES \\
\hline 7 & Biswas & $\begin{array}{c}\text { Lattice Boltzman Method/Very Large Eddy } \\
\text { Simulation with wall model (LBM/VLES) }\end{array}$ \\
\hline 8 & Noelting et al. \\
\hline
\end{tabular}

Table 3-2 Summary of previous work performed using other turbulence models

Saric et al. [8, 36] assessed a number of computational models viz. LES, DES [4, 37] , Delayed DES (DDES) [38] and a zonal hybrid LES/RANS [39] (HLR) scheme. They performed the solution of both 3D filtered and RANS equations on a block-structured body fitted collocated 
mesh, using a second order central scheme for spatial discretization and second order Crank Nicolson scheme for time advancement. The subgrid scales were modeled using the standard Smagorinsky [40] model. The DES and DDES methods applied are the original versions of Spalart-Allmaras' one equation model [5]. In the DES method the original Spalart Allmaras' one equation is applied to model the small eddies in the attached boundary layer, while the larger detached eddies typically seen in wakes and separated region are simulated using LES. Smaller eddies seen in the said region are still modeled, but their influence is much smaller than the boundary layer eddies. The switch between LES and RANS regions are made based on a length scale whose value is calculated based on local grid spacing and distance from wall. In the Delayed Detached Eddy Simulation method, the switch between the LES and RANS regions are made based on not only the length scale mentioned above and but also the eddy viscosity field through a discriminator function. This is required when some regions of the grid is "ambiguous" to DES, or in other words, is spaced such that it calls for switching to the LES as per the original DES formulation, but is not fine enough for the same. This can happen due to number of reasons such as grid refinement, thick boundary layers etc. The new zonal two-layer hybrid LES/RANS (HLR) model proposed by the authors themselves employs an eddy viscosity RANS model in the near wall region with an LES in the outer layer. The Smagorinsky model [40] was employed for the LES region and a $\mathrm{k}-\varepsilon$ model which solves the equation for the homogeneous dissipation $\varepsilon^{h}$ (Jakrilic and Hanjalic [41]) were considered in the wall vicinity.

The calculations were performed on a mesh of size $426 \times 145 \times 64$ cells for the LES and $426 \times 145 \times 32$ cells for the HLR method. The DES was performed on a grid of size $426 \times 145 \times 28$ cells. In all computations experimental mean velocity profile without the fluctuations were 
prescribed at the inlet plane placed at $2.14 \mathrm{c}$ upstream of the hump. The DES, LES and HLR methods predicted a reattachment length of $1.121,1.114$ and 1.183 respectively. The plot of streamwise pressure coefficient was in close agreement with the experiment for all cases. The mean velocities were predicted with good accuracy except in the case of HLR for certain locations. In case of turbulent shear stresses, LES, DES and HLR over predicted the experimental values at locations upstream of separation. $(\mathrm{x} / \mathrm{c}=0.65$ and 0.66$)$. DES still over predicted in the re-circulation region, while LES and HLR agreed better. For the locations downstream of reattachment, DES agreed better with experiment while LES and HLR under predicted the experimental data.

Lynos et al. [42] evaluated DES, DDES (Spalart [38]) and Zonal DES (Slimon [43]) for this case. The zonal DES (ZDES) works much like the DDES, except that it uses a different discriminator function to distinguish between the RANS and LES regions. They used Acusolve, a commercial finite element flow solver that is second order accurate in space and time, on an unstructured mesh. Their calculation yielded reattachment locations of $1.36,1.18$ and 1.18 for the DES, ZDES and DDES methods respectively. For the mean velocity profiles, the DES showed poor agreement with the experiment, while the DDES and ZDES methods exhibited good agreement. In case of streamwise pressure coefficient, the DES under-predicted the first suction peak while over predicted the second. Other methods fail to predict the suction peak near the separation point accurately. All three methods under predict the turbulent kinetic energy.

Morgan et al. [44] performed an implicit LES (ILES) for this problem, however, at a reduced Reynolds number of $2 \times 10^{5}$. ILES methods capture the inviscid energy cascade in the inertial range and rely on their own intrinsic numerical dissipation to act as subgrid stress model. Their 
computational grid had a size of $738 \times 151 \times 165$ points, and the inlet for the domain was placed at $\mathrm{x} / \mathrm{c}=-1.2$. For the inlet boundary conditions, results from a spatially evolving flat plate LES were used. The ILES used a higher order non-dispersive spatial filter in lieu of a sub-grid stress model. As for the results, they observed reattachment at location of $\mathrm{x} / \mathrm{c}=1.14$. For the streamwise pressure coefficient, the LES under-predicted the first suction peak and accurately predicted the second peak near separation by accounting for the effect of end plate blockage in the computational domain. The LES over predicted the skin friction at the leading edge of the hump, and then under predicted over the mid span. The agreement between experiment and LES improved once the flow separated. The mean velocity profiles obtained from their LES did not show a very accurate prediction of the experimental data. As for the turbulence data, there was consistent over prediction for all parameters at location $\mathrm{x} / \mathrm{c}=0.65$. The agreement between the computation and experiment was better at locations $\mathrm{x} / \mathrm{c}=0.8,1.0$ and 1.2 with respect to the trend and peak values, except for $\overline{v^{\prime} v^{\prime}}$, which showed over prediction everywhere.

Frank et al [45] performed compressible LES for this case over a range of mach numbers from 0.1 to 0.6 . The computations were performed on a grid of size $800 \times 160 \times 64$ points. The inflow turbulent boundary layer was not fully resolved and instead, the boundary layer profile at the wall is initialized with velocity perturbations formulated with sums of random Fourier modes. The results show discrepancy with experiment in mean velocity profiles, although the agreement with experiment is better for the Reynolds stresses. However the difference between the computed Reynolds stress profiles and experimental profile is highest in the recirculation region, an observation different from the other works. 
You et al. [46] employed LES with a dynamic subgird-scale model [47] with modification proposed as in [48] using an energy conservative second order central difference scheme on a staggered mesh. The computations were performed on a mesh of size $721 \times 161 \times 65$. The inflow boundary layer data were provided from a separate simulation of a flat plate boundary layer using the method of Lund et al [49]. For the results, they obtained a reattachment length of 1.09. The variation of streamwise surface pressure coefficient show good agreement with experiment, especially in the prediction of the two suction peaks. The skin friction coefficient is over predicted in the region from the leading edge of the hump up to the separation point, after which it agreed with the experiment. The mean velocity, turbulent kinetic energy and turbulent shear stress profiles showed good agreement with the experiment.

Avdis et al. [50] performed an implicitly filtered LES on a co-located mesh of size 768x96x128 cells. The dynamic Smagorinsky model approximated the sub-grid scale stresses. Central differencing scheme was used for approximating the fluxes and a minimally diffusive wiggle detector was used to eliminate odd-even oscillations. The inflow velocity profile was generated using the method of Lund et al. [49]. The computational domain had made adjustments to account for the blockage effects of the end plate. For the results, they obtained a reattachment length of 1.079. The plot of surface pressure coefficient shows good agreement with the experiment. The mean velocity profiles agree well with the experimental results, however, the profiles of turbulent parameters show over prediction of the data from the experiment at all reported locations.

Biswas [51] performed a higher order LES on a grid of size $1171 \times 291 \times 165$ points for a computational domain that extended a length of 5.2 units in streamwise direction and 0.2 units in 
the spanwise direction. The inlet boundary condition was obtained from a precursor simulation. The plot of streamwise variation of $\mathrm{C}_{\mathrm{P}}$ slightly under predicted the first suction peak, while accurately predicted the suction peak near separation point. However, the work does not mention whether the computational domain accounted for the end wall blockage. The reattachment location was obtained as 1.115 .

Noelting et al. [52] used the Lattice Boltzman Method (LBM), coupled with a very large eddy simulation turbulence model (VLES). Since a direct solution of the lattice Boltzmann equations would be similar to a DNS calculation, turbulence modeling was incorporated into the governing equations by modifying the relaxation time to give an extended relaxation time scale. The extended relaxation time was then calculated using a variant of the RNG k- $\varepsilon$ equation. In addition, the equations were further modified by incorporating swirl correction, which allowed the resolution of unsteady large-scale vortices in the regions where they could be resolved with the underlying grid. Thus by this method certain regions of the flow are modeled in a RANS fashion while other regions are resolved in a LES-like or VLES-like methodology. A wall model was employed in this study, in which the function reduced to a linear profile if the first grid point was in the viscous sublayer, or to a log-law profile, if the first grid point was in the log layer. The complete geometry including the end plates was modeled, with the computational grid consisting of 23 million voxels. The simulation predicted the profile of $\mathrm{C}_{\mathrm{P}}$ accurately, although the reported mean velocity profiles showed some discrepancies with the experiment. As for the turbulence data, only the TKE at location $\mathrm{x} / \mathrm{c}=0.8$, which was in the recirculation region, was reported, and it showed good agreement with the experiment. The reattachment location was found to be $\mathrm{x} / \mathrm{c}=$ 1.13 
To summarize in general, the prediction of reattachment point is better achieved through LES than RANS models. Once again, accounting for the end plate blockage in the geometry is seen to improve the prediction of suction peak near separation in the profile of $\mathrm{C}_{\mathrm{P}}$. There is inconsistency in the accuracy of the calculated mean profiles between different works while the Reynolds stresses show discrepancy with experiments in most cases. In general, the Reynolds stresses are best predicted in the recirculation region, with a couple of exceptions. 


\section{Computational Setup}

Large Eddy Simulation resolves large scale energy containing turbulent eddies that are threedimensional in nature. It is therefore necessary to perform a three dimensional calculation, in spite of the flow being nominally two-dimensional, to capture the appropriate flow physics. Figure 4-1 shows the calculation domain.

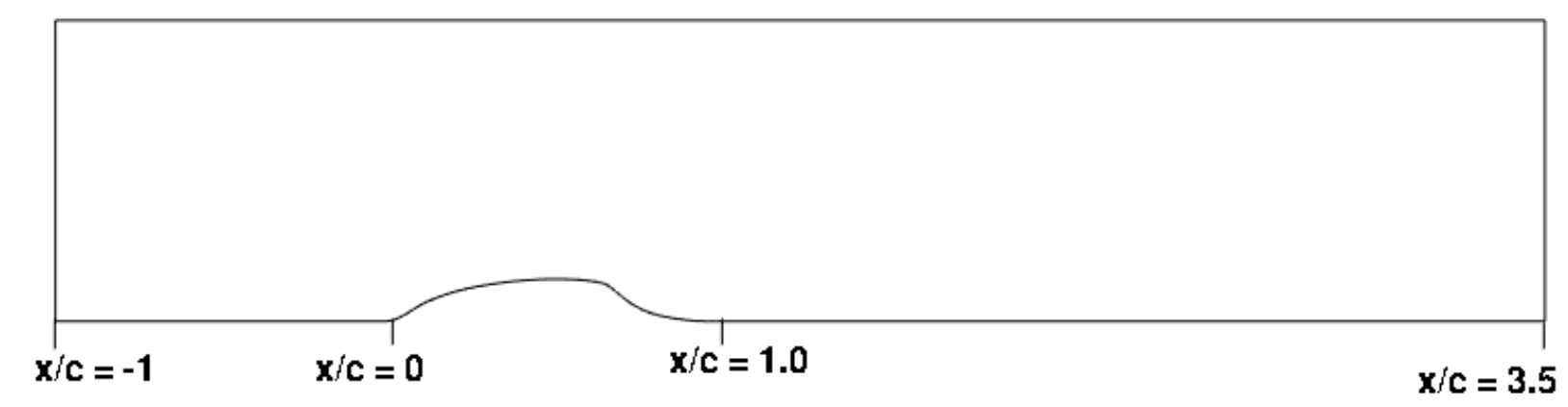

Figure 4-1: Computational Domain

The computational domain is three-dimensional with the spanwise direction assumed periodic (homogeneous) without the sidewalls. The inlet is at $\mathrm{x} / \mathrm{c}=-1$ where $\mathrm{x} / \mathrm{c}=0$ is the base of the hump. Although the boundary conditions from the experiment are provided at the location $\mathrm{x} / \mathrm{c}$ $=-2.16$, the inlet is placed at $\mathrm{x} / \mathrm{c}=-1$ so as to reduce the computational expenses. A length of 2.5 times the chord is provided downstream of the hump. The height of the domain is set at $0.91 \mathrm{c}$, where $\mathrm{c}$ is the chord. This is in accordance with the height of the experimental set up. The spanwise length is chosen to be $0.156 \mathrm{c}$, which is approximately 1.2 times the height of the hump. It was reported by You et al. [46] that the results were insensitive to increasing the spanwise domain size over $0.15 \mathrm{c}$. The computational domain thus has an overall size of $4.5 \mathrm{c} \times 0.91 \mathrm{c} \mathrm{x}$ $0.156 \mathrm{c}$ in the $\mathrm{x}, \mathrm{y}$ and $\mathrm{z}-$ direction. 


\subsection{Details of the Grid}

The Reynolds number of the flow is of the order of 1 million. Accurate prediction of flow field in such high Reynolds number flows requires very fine grids. This work performed a wall resolved LES (WRLES) as well as a Wall modeled LES (WMLES) for the flow over the hump. A WRLES requires the first grid point to be placed at a $\mathrm{y}^{+}<1$ for accurate prediction. In case of WMLES, the wall model allows the first off-wall grid point to be placed at a $\mathrm{y}^{+}<50$. Hence different grids are used for the WRLES and WMLES calculations, with the WRLES and WMLES grids having 161 points and 129 points respectively in the y-direction. The $\mathrm{y}^{+}$of the first off-wall grid point remains less than 1 for the WRLES grid and varies from 20 - 40 for the WMLES grid, for the entire length of the domain.

Figure 4-2 shows the streamwise variation of $\Delta \mathrm{x}^{+}, \Delta \mathrm{y}^{+}$(first node off wall) and $\Delta \mathrm{z}^{+}$for the grid configurations used for the calculations. The $\mathrm{y}^{+}$shown in the figure is for the grid used for WMLES and it can be seen that the value has been kept within the range of $20-40$ for the entire length of the domain. The case that did not employ the wall model was also performed on this grid. In case of the WRLES, which is not shown, the $\mathrm{y}^{+}$is maintained below 1 for the entire length of the domain, while the distribution in $\mathrm{x}$ and $\mathrm{z}$-direction are the same as shown in the figure. The resolution in $\mathrm{x}$ and $\mathrm{z}$ is kept coarse because of the fact that the turbulent structures outside of the inner boundary layer $\left(\mathrm{y}^{+<50-100)}\right.$ have a larger footprint. Hence, in the region between $0<\mathrm{x} / \mathrm{c}<1.4, \Delta \mathrm{x}^{+}$has a maximum value of 200 and is comparable to $\Delta \mathrm{y}^{+}$of the WMLES grid, in the region of interest $0.65<\mathrm{x} / \mathrm{c}<1.3$, despite having a coarse distribution elsewhere. In

the recirculation region the maximum value for $\Delta x^{+}$is approximately 50 . In case of $\Delta z^{+}$, a 
uniform distribution of 64 cells in $\mathrm{z}$ direction yields a maximum value of approximately 90 , and stays around the value of 50 for most of the recirculation region

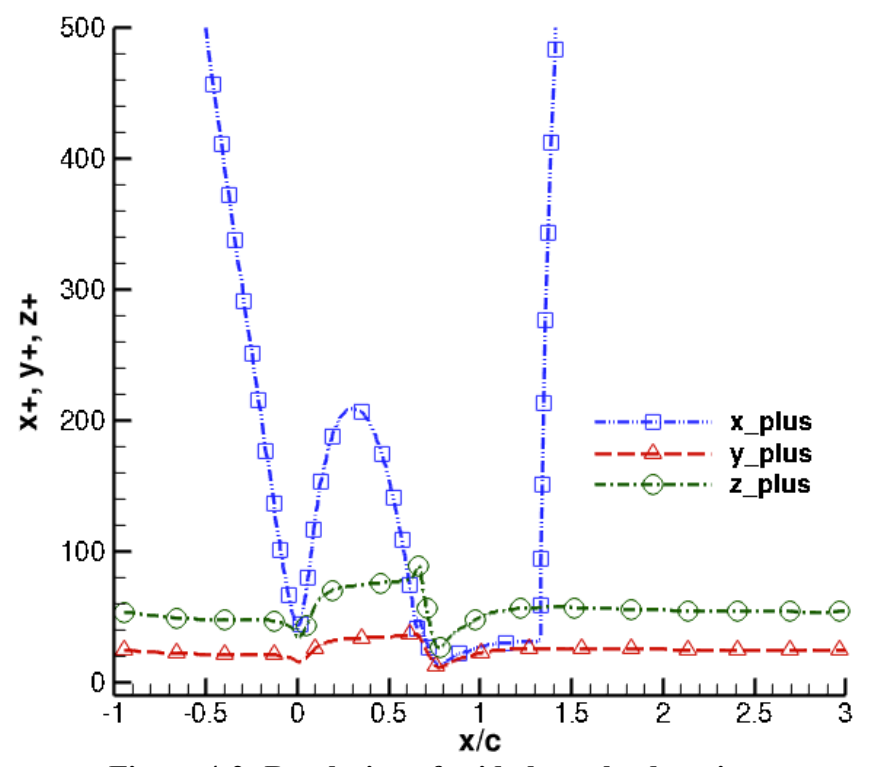

Figure 4-2: Resolution of grid along the domain

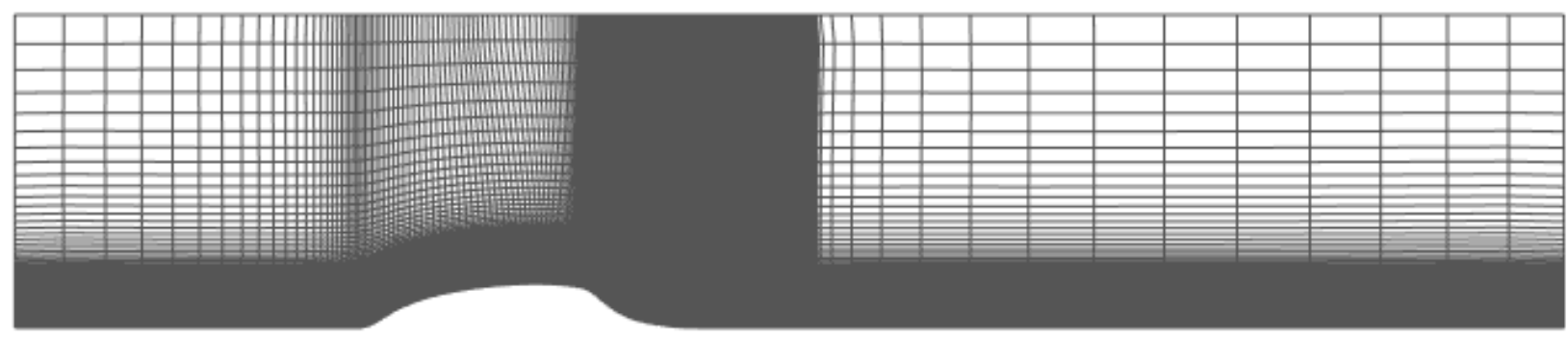

Figure 4-3: Computational grid; every $3^{\text {rd }}$ line shown in $x$, every $2^{\text {nd }}$ line shown in $y$

Figure 4-3 shows the overall computational grid used for the WMLES calculation with every $3^{\text {rd }}$ line and every $2^{\text {nd }}$ line shown in $\mathrm{x}$ and $\mathrm{y}$ - direction respectively. In both WRLES and WMLES grids, there are a total of 792 cells in the $\mathrm{x}$ - direction and 64 cells in the $\mathrm{z}$ - direction. Thus the WRLES grid has an overall size of $792 \times 160 \times 64$ cells and the WMLES grid has an overall size 
of $792 \times 128$ x 64 cells. The observations from a grid refinement study, as well as a calculation performed on grid with $792 \times 128 \times 32$ cells will also be presented in chapter 6 .

\subsection{Details of the simulation}

The experiment measured a fully developed turbulent boundary layer at $\mathrm{x} / \mathrm{c}=-2.16$. The experimental data includes the mean and rms profiles of streamwise velocity at the said location and the mean profile can be seen to obey the $1 / 7^{\text {th }}$ power law for turbulent boundary layer. The boundary layer thickness at $\mathrm{x} / \mathrm{c}=-2.16$ was used to find the origin $(\mathrm{x}=0)$ assuming a fully turbulent boundary layer using the expression

$$
\mathrm{X}=\frac{\mathrm{U}_{\infty}^{1 / 4}}{v^{1 / 4}} \frac{\delta^{5 / 4}}{0.37^{5 / 4}}
$$

The boundary layer thickness at the computational domain inlet $(\mathrm{x} / \mathrm{c}=-1.0)$ was calculated using this distance from the origin. Using this new value of $\delta$, a new steady smooth velocity profile

was generated using the $1 / 7^{\text {th }}$ power law. This velocity profile was supplied at the inlet face of the domain as the boundary condition without any unsteady turbulent fluctuations. The impact of supplying a steady smooth profile instead of a turbulent profile as boundary condition on the accuracy of the results shall be presented later.

All the calculations were performed for 4.5 non-dimensional time units to allow the flow to convect through the entire length of the domain before the averaging for turbulent quantities began. The calculations were performed for a further 9 non-dimensional time units for obtaining the time averaged data. In case of WRLES, the grid was split into 66 blocks to be distributed across 66 processors, while the WMLES grid was split into 44 blocks to be distributed across 44 processors. It was observed that the WRLES required approximately 33,000 CPU hours to complete, whereas the WMLES required approximately 6400 CPU hours only. For the WMLES 
on the grid of size $792 \times 128 \times 32$, the CPU time was further reduced to about 3200 hours. This indicates reduction in computational time by approximately an order of magnitude.

\subsection{Numerical Method}

This work was performed using GenIDLEST [53], a general purpose turbulent fluid flow and heat transfer solver capable of performing LES, DES and RANS-URANS calculations. The code uses a hybrid body-fitted structured mesh $(\xi, \eta, \zeta$-coordinates $)$ with an unstructured block topology. The code is parallelized and exhibits excellent scalability with MPI and OpenMP and is routinely used for LES on grids of the $\mathrm{O}\left(10^{6}\right)$ to $\left(10^{7}\right)$ on $\mathrm{O}(100)$ processing cores. This section presents a theoretical description of the WRLES and WMLES employed for the calculations in this work. The numerical formulation of the LES equations that are solved in GenIDLEST from the governing equations of fluid flow is given first. Following this, a brief description of the generalized two-layer model for complex geometries developed by Patil and Tafti [15] is also provided.

\subsubsection{Governing Equations and sub-grid stress modeling}

The governing equations for the flow over the wall-mounted hump are the mass and momentum equations of the unsteady incompressible Navier-Stokes equations. The equations are

non-dimensionalized by suitable a length scale $\left(\mathrm{L}^{*}\right)$ and a velocity scale $\left(\mathrm{U}^{*}\right)$, which for the present case would be the chord length of the hump and the free stream velocity respectively. The non- dimensional equations are mapped from the physical space to computational space by a boundary conforming transformation $\mathrm{x}=\mathrm{x}(\xi)$ where $\mathrm{x}=\mathrm{x}(\mathrm{x}, \mathrm{y}, \mathrm{z})$ and $\xi=\xi(\xi, \eta, \zeta)$ to take the following conservation form. 
Mass:

$$
\frac{\partial}{\partial \xi_{j}}\left(\sqrt{g} U^{j}\right)=0
$$

Momentum:

$$
\frac{\partial}{\partial T}\left(\sqrt{g} u_{i}\right)+\frac{\partial}{\partial \xi_{j}}\left(\left(\sqrt{g} U^{j}\right) u_{i}\right)=-\frac{\partial}{\partial \xi_{j}}\left(\sqrt{g}\left(\vec{a}^{j}\right)_{i} p\right)+\frac{\partial}{\partial \xi_{j}}\left(\left(\frac{1}{\operatorname{Re}}+\frac{1}{\operatorname{Re}_{t}}\right) \sqrt{g} g^{j k} \frac{\partial u_{i}}{\partial \xi_{k}}\right)
$$

where $\vec{a}^{j}$ are the contravariant basis vectors, $\sqrt{g}$ is the Jacobian of the transformation, $g^{j k}$ are the elements of the contravariant metric tensor, $\sqrt{g} U^{j}=\sqrt{g}\left(\vec{a}^{j}\right)_{k} u_{k}$ is the contravariant flux vector, $u_{i}$ is the Cartesian velocity vector and $\mathrm{p}$ is the pressure. $T^{*} U^{*} / L^{*}$ is the non-dimensional time and the Reynolds number is given by $U^{*} L^{*} / v, \mathrm{Re}_{\mathrm{t}}$ is the inverse of the subgrid eddyviscosity which is modeled as

$$
\frac{1}{\operatorname{Re}_{t}}=C_{s}^{2}(\sqrt{g})^{2 / 3}|\bar{S}|
$$

where $|\bar{S}|$ is the magnitude of the strain rate tensor given by $|\bar{S}|=\sqrt{2 \overline{S_{i k} S_{i k}}}$ and the Smagorinsky constant $C_{s}^{2}$ is obtained via the dynamic subgrid stress model [47]. To this end, a second test filter, denoted by $\hat{G}$ with characteristic length larger than the grid filter $\bar{G}$ is applied to the filtered governing equations. The test-filtered quantity is obtained from the grid-filtered quantity by a second order trapezoidal given as $\hat{\phi}=\frac{1}{4}\left(\bar{\phi}_{i-1}+2 \bar{\phi}_{i}+\phi_{i+1}\right)$ in one dimension. The resolved turbulent stresses, representing the energy scales between the test and grid filters, $L_{i j}=\widehat{\bar{u}_{\imath} \bar{u}_{\jmath}}-$ $\widehat{\overline{u_{l}}} \widehat{\overline{u_{j}}}$ are then related to the subtest, $T_{i j}=\widehat{\widehat{u_{l} u_{\jmath}}}-\widehat{\overline{u_{l}}} \widehat{\overline{u_{\jmath}}}$, and subgrid - scale stresses, $\tau_{i j}=\overline{u_{i} u_{j}}-\overline{u_{i}} \overline{u_{j}}$, through the identity, $L_{i j}^{a}=T_{i j}^{a}-\bar{\tau}_{i j}^{a}$. The anisotropic subgrid and subtest-scales 
are then formulated in terms of the Smagorinsky eddy viscosity model as:

$$
\begin{gathered}
\widehat{\tau_{l \jmath}^{a}}=-2 C_{s}^{2}(\sqrt{g})^{\frac{2}{3}} \widehat{\bar{S} \mid \overline{S_{l \jmath}}} \\
T_{i j}^{a}=-2 C_{s}^{2} \alpha(\sqrt{g})^{2 / 3}|\hat{\bar{S}}| \widehat{S_{l \jmath}}
\end{gathered}
$$

using the identity,

$$
\widehat{L_{l j}^{a}}=\widehat{L_{l j}}-\frac{1}{3} \delta_{i j} L_{k k}=2 C_{s}^{2}(\sqrt{g})^{\frac{2}{3}}\left[\alpha|\hat{\bar{S}}| \hat{\overline{S_{i j}}}-\widehat{|\bar{S}| \overline{S_{l j}}}\right]=-2 C_{s}^{2}(\sqrt{g})^{\frac{2}{3}} M_{i j}
$$

where $\alpha$ is the ratio of the characteristic length scale associated with the test filter to that of the grid filter. For a representative one-dimensional test filtering operation here, $\alpha$ is taken to be $\left[\widehat{\overline{\Delta_{l}}} / \overline{\Delta_{l}}=\sqrt{6}\right]$. Using the least-squares minimization procedure of Lilly [48], a final expression for $C_{s}^{2}$ is obtained as:

$$
C_{S}^{2}=-\frac{1}{2} \frac{1}{\sqrt{g}^{2 / 3}} \frac{L_{i j}^{a} \cdot M_{i j}}{M_{i j} \cdot M_{i j}}
$$

where the value of $C_{s}^{2}$ is constrained to be positive for numerical stability.

\subsubsection{Discretization and Solution Algorithm}

The second order central scheme (SOC) for spatial discretization is chosen due to its minimally dissipative nature. The governing equations for the conservation of mass and momentum mentioned above are discretized in space using a conservative finite volume approach on a collocated grid topology, which implies that the Cartesian velocities and pressure are calculated and stored at the cell center, whereas the contravariant fluxes are calculated and stored at the cell faces. The discretized continuity and momentum equations are advanced in time 
using a projection method where the time integration happens in two steps. The first step, called the predictor step, calculates an intermediate velocity field and the second step or the corrector step updates the velocity for the new time step by enforcing continuity. Mathematically, these can be represented a follows,

\section{Predictor Step :}

$$
\frac{\sqrt{g}^{n+1} \widetilde{u_{i}}-\sqrt{g}^{n} u_{i}^{n}}{\Delta t}=D_{i}-C_{i}
$$

where $D_{i}$ stands for the diffusion terms and $C_{i}$ stands for the convection terms. Both the convection and diffusion terms are treated implicitly by a Crank-Nicolson scheme.

\section{Convection terms}

$$
\begin{gathered}
C_{i}=\frac{\partial}{\partial \xi_{j}}\left(\sqrt{g} U^{j} u_{i}\right)^{n+\frac{1}{2}} \\
C_{i}=\frac{1}{2}\left[\frac{\partial}{\partial \xi_{j}}\left(\sqrt{g} U^{j} u_{i}\right)^{n}+\frac{\partial}{\partial \xi_{j}}\left(\sqrt{g}\left(U^{j}\right)^{n+1} \widetilde{u_{l}}\right)\right]
\end{gathered}
$$

The contravariant fluxes at time level $n+1$ are linearized using a two-step ( $n$ and $n-1$ time level) second order extrapolation as:

$$
\sqrt{g} U^{j n+1}=2 \sqrt{g} U^{j n}-\sqrt{g} U^{j n-1}
$$

\section{Diffusion terms}

$$
D_{i}=\frac{1}{2}\left[\frac{\partial}{\partial \xi_{j}}\left(\left(\frac{1}{R e}+\frac{1}{R e_{t}}\right) \sqrt{g} g^{j k} \frac{\partial u_{i}^{n}}{\partial \xi_{k}}\right)+\frac{\partial}{\partial \xi_{j}}\left(\left(\frac{1}{R e}+\frac{1}{R e_{t}}\right) \sqrt{g} g^{j k} \frac{\partial \widetilde{u_{l}}}{\partial \xi_{k}}\right)\right]
$$




\section{Corrector step}

The corrector step is performed once the intermediate velocity field is calculated from the predictor step. In this step, the continuity equation is used to derive the pressure equation, which is solved to obtain the pressure field at time level $(n+1)$. Described below is the procedure employed in deriving the pressure equation.

The intermediate cell face contravariant fluxes are constructed first as follows

$$
\sqrt{g} \widetilde{U^{j}}=\sqrt{g}\left(\vec{a}^{j}\right)_{i} \widetilde{u_{\imath}}
$$

The correction form of the cell centered Cartesian velocities and cell face contravariant fluxes are then written as:

$$
\begin{aligned}
u_{i}^{n+1} & =\widetilde{u}_{\imath}-\Delta t\left(\vec{a}^{j}\right)_{i} \frac{\partial p^{n+1}}{\partial \xi_{j}} \\
\sqrt{g}\left(U^{i}\right)^{n+1} & =\sqrt{g} \widetilde{U}^{i}-\Delta t \sqrt{g} g^{i k} \frac{\partial p^{n+1}}{\partial \xi_{k}}
\end{aligned}
$$

Equation (4.16) in combination with the continuity equation (4.2) is then used to derive the pressure equation, which takes the following form.

$$
\frac{\partial}{\partial \xi_{j}}\left(\sqrt{g} g^{j k} \frac{\partial p^{n+1}}{\partial \xi_{k}}\right)=\frac{1}{\Delta t} \frac{\partial \sqrt{g} \widetilde{U}^{j}}{\partial \xi_{j}}
$$

The use of contravariant fluxes at the cell faces in constructing the pressure equation enables this method to emulate a staggered grid arrangement. The pressure field at time level $n+1$ is then used to correct the nodal Cartesian velocities and the cell face contravariant fluxes using equation (4.15) and equation (4.16) respectively. 


\subsubsection{Parallel Implementation}

The computational code is developed in a multi-block framework to facilitate parallelization and engages multiple processors simultaneously. The computational domain is hence divided into blocks, with each processor handling one or more blocks. The total number of blocks limits the maximum number of processors. Each block has a separate data structure, with the data transmitted across processors through the Message Passing Interface (MPI). Each computational block is further divided into virtual cache blocks while solving the linear systems. Details of the software architecture of GenIDLEST can be found in Tafti [53].

\subsubsection{Two-layer Wall Model}

The two-layer wall model used in the present case is described in detail in [15] and is briefly summarized here. The wall model solves a reduced set of simplified equations on a virtual grid along normal between the first off-wall grid point $\left(\mathrm{y}^{+}<50\right)$ and the wall. The instantaneous flow velocity in the outer layer is supplied as a boundary condition to the inner layer where the wall shear stress is calculated from the solution of the reduced set of equations. The calculated wall shear stress is then provided as a boundary condition for the solution of the outer layer equations at the first off-wall node. This procedure thus accomplishes the coupling between the inner and outer layer.

The fact that the normal and tangential velocities are aligned with the $\mathrm{x}, \mathrm{y}$ and $\mathrm{z}$-direction in Cartesian geometries makes the implementation of two-layer wall modeling straightforward in the same. However, in case of a general body fitted non-orthogonal grid, the local $(\xi, \eta, \zeta)$ coordinates need not necessarily be aligned with the normal and tangential direction at the wall, 
the physical coordinate system $(\mathrm{x}, \mathrm{y}, \mathrm{z})$ or the Cartesian velocities $(\mathrm{u}, \mathrm{v}, \mathrm{w})$. Hence special considerations were given for the development of a generalized wall model.

To this effect, the equations are formulated in local wall coordinates $(n, t)$ within the inner layer. Reduced dimensionality boundary layer type equations are written for the transport of normal and tangential momentum in the inner layer as shown below instead of three separate equations for each of the velocity components.

$$
\begin{gathered}
\frac{\partial u_{t}}{\partial t}+\frac{\partial u_{n}}{\partial n}=0 \\
\frac{\partial u_{t}}{\partial T}+\frac{\partial\left(u_{t} u_{n}\right)}{\partial n}+\frac{\partial\left(u_{t}^{2}\right)}{\partial t}=-\frac{\partial P}{\partial t}+\frac{\partial}{\partial n}\left[\left(\frac{1}{R e}+\frac{1}{R e_{t}}\right) \frac{\partial u_{t}}{\partial n}\right]+S_{u}
\end{gathered}
$$

with $\mathrm{u}_{\mathrm{t}}=\mathrm{u}_{\mathrm{n}}=0$ at the wall, $\mathrm{u}_{\mathrm{t}}=\left\|\overrightarrow{U_{t}}\right\|$ and $\mathrm{u}_{\mathrm{n}}=\left\|\overrightarrow{U_{n}}\right\|$ at the edge of the inner layer. Here, the tangential pressure gradient is assumed constant in the inner layer and is calculated at the first off-wall grid point. Neglecting the time derivative and convection terms reduces the number of independent variables to one spatial dimension (n), allowing solution of tri-diagonal system of equation at each station along the normal to the wall

$$
\frac{\partial}{\partial n}\left(\frac{1}{R e}+\frac{1}{R e_{t}}\right) \frac{\partial u_{t}}{\partial n}=\frac{\partial P}{\partial t}
$$

The eddy viscosity is modeled by [54]

$$
\begin{aligned}
\frac{1}{\operatorname{Re}_{t}} & =\frac{\kappa}{\operatorname{Re}} d^{+}\left(1-e^{-d^{+} / A}\right)^{2} \\
d^{+} & =\rho u_{\tau} d / \mu
\end{aligned}
$$




$$
\mathrm{u}_{\tau}=\sqrt{\left\|\tau_{\mathrm{w}}\right\| / \rho}
$$

Where $\kappa$ is the von Karman constant, $d$ is the normal distance from wall and $A=19$. Equation (4.20) is solved on a virtual grid that extends between the wall node and the first off-wall node. Figure 4-4 shows the virtual grid embedded between the LES grid.

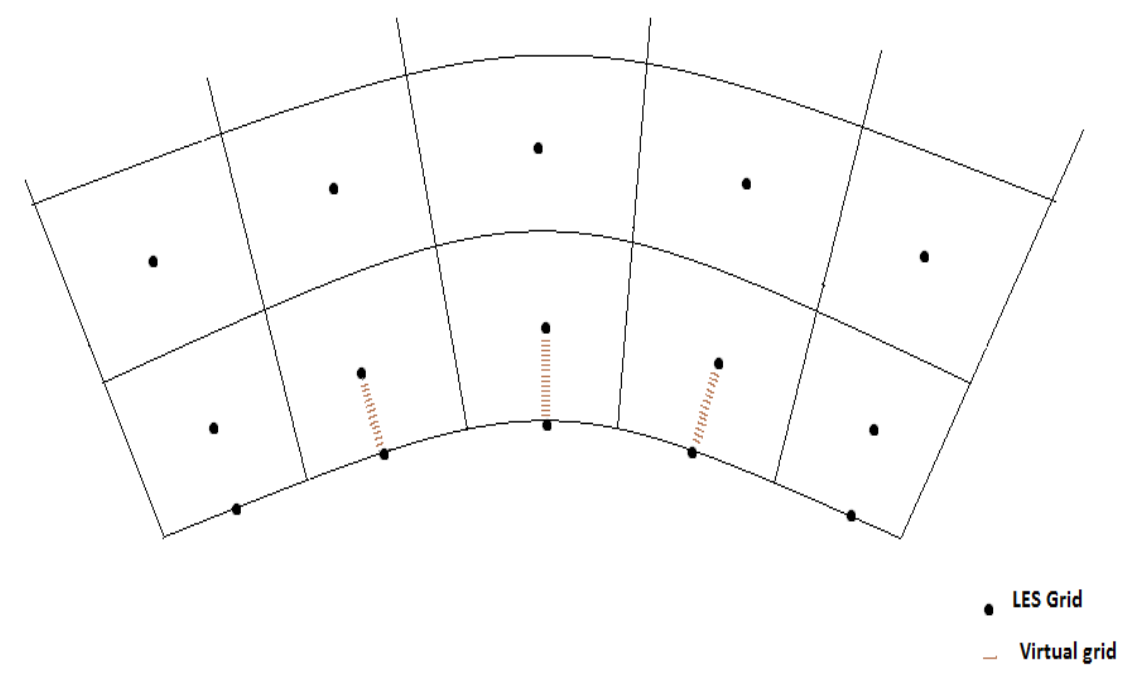

Figure4-4: Virtual grid embedded between the LES grid

The virtual grid consists of 64 equally placed nodes between the wall node and the first node off wall. The equation is solved iteratively for $u_{\tau}$ and hence $\tau_{w}$ using a standard tridiagonal solver for a second order central difference approximation. From the solution of equation (20), the magnitude of tangential shear stress is calculated as

$$
\left\|\tau_{\mathrm{w}}\right\|=\left.\left(\frac{1}{\mathrm{Re}}+\frac{1}{\mathrm{Re}_{\mathrm{t}}}\right) \frac{\partial \mathrm{u}_{\mathrm{t}}}{\partial \eta}\right|_{\text {wall }}
$$

which is then decomposed into the respective directional components as

$$
\vec{\tau}_{w}=\left\|\tau_{w}\right\| \vec{t}=\left\|\tau_{w}\right\| t_{x} \vec{e}_{x}+\left\|\tau_{w}\right\| t_{y} \vec{e}_{y}+\left\|\tau_{w}\right\| t_{z} \vec{e}_{z}
$$


where $\vec{t}$ is the unit tangential vector and

$$
\left.\left(\frac{1}{R e}+\frac{1}{R e_{t}}\right) \frac{\partial u_{i}}{\partial \eta}\right|_{\text {wall }}=\left\|\tau_{w}\right\| t_{i}
$$

The calculated wall shear stress is then incorporated into the discretized momentum equations (3) at the first off-wall node in the outer layer. 


\section{Results}

The computational domain is shown below in figure 5-1 with locations marked where the experimental data is available. The available experimental data include mean velocity profiles and Reynolds stresses at all the locations marked in the figure below, and the variations of pressure coefficient and skin friction in the streamwise direction.

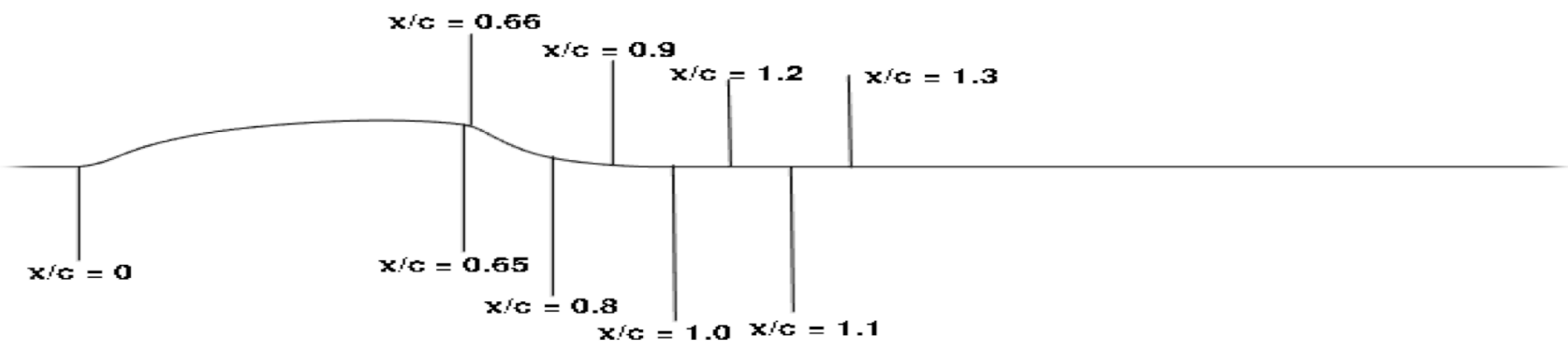

Figure 5-1: Computational domain

This chapter has four sections. The first section presents results from WMLES performed on a grid of size $792 \times 128 \times 64$ cells in the $\mathrm{x}, \mathrm{y}$ and $\mathrm{z}$-directions respectively. The results are compared to those obtained from the same grid when the wall model was not used. The second section describes the observations from a grid refinement study that was performed to understand the possible influence of the grid resolution on the results. The third section details the results obtained from a WRLES, which are also compared to those obtained from the wall-modeled simulations. The final section takes a look into the velocity profile generated by the wall model in the virtual grid between the wall and first cell, and compares it with the actual velocity profile obtained from LES at the same location. 
As mentioned earlier, all calculations were performed for 4.5 non-dimensional time units before beginning averaging for turbulent quantities, and a further 9 non-dimensional time units for obtaining the time averaged data. Additional averaging was conducted in the homogenous spanwise direction to increase the statistical sample size.

\subsection{Wall Modeled LES}

\subsubsection{Incoming boundary layer}

As mentioned earlier, a steady, smooth mean velocity profile using the $1 / 7^{\text {th }}$ power law without any turbulent fluctuations was specified at $\mathrm{x} / \mathrm{c}=-1$. This emulated the experimental conditions, which measured a fully turbulent boundary layer at $\mathrm{x} / \mathrm{c}=-2.16$. In the calculations, the boundary layer thickness at the inlet $(\mathrm{x} / \mathrm{c}=-1)$ was found to be 0.089 non-dimensional units by the procedure mentioned in the previous chapter. Figure 5-2 shows the degree of unsteadiness in the computed boundary layer as represented by the instantaneous coherent vorticity [55]

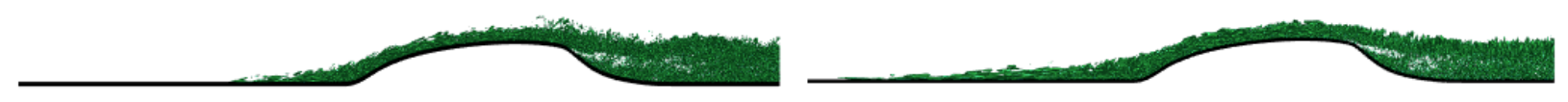

Figure 5-2: Left: WMLES, Right: WRLES

In both cases (WMLES and WRLES), it can be seen that the turbulent structures have developed in the boundary layer before the flow reached the hump. It can also be noted that in case of WRLES, the structures start developing earlier than in the WMLES. These figures 
indicate that both WRLES and WMLES develop into an unsteady turbulent boundary layer in spite of the steady, smooth mean velocity supplied at the inlet as boundary condition.

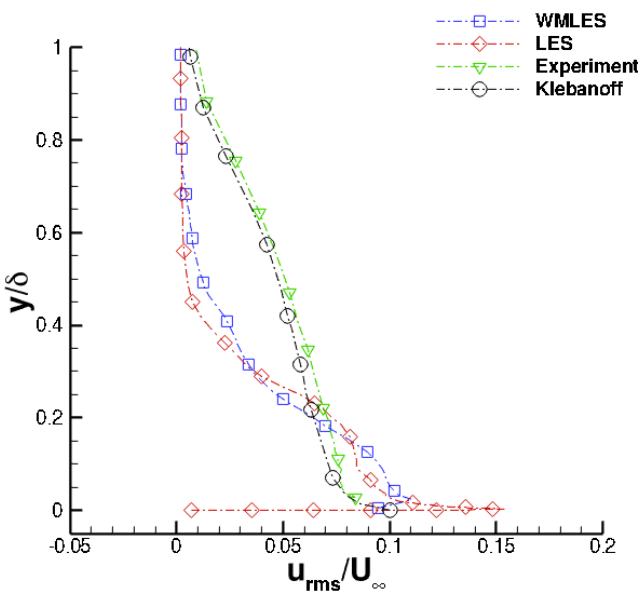

Figure 5-3: Boundary layer at $\mathbf{x} / \mathbf{c}=\mathbf{- 0 . 1}$

Figure 5-3 shows the normalized profiles of $U_{\text {rms }}$ at the location $\mathrm{x} / \mathrm{c}=-0.1$, where $\mathrm{x} / \mathrm{c}=0$ is the base of the hump, for both WMLES and WRLES calculations. Also shown are the profiles observed experimentally and the measurements by Klebanoff [56] for a fully turbulent boundary layer. It can be seen that the boundary layer has developed turbulent fluctuations, with the peak value of $U_{\text {rms }}$ matching closely with the data from experiment and that of Klebanoff [56] in case of WMLES. In case of LES, the peak is slightly higher. In both cases, the profile matches with the experimental profile up to the point where the $y / \delta=0.3$, beyond which it shows under prediction. This could have been improved if the inlet was placed farther away which would allow a larger length for the turbulent fluctuations to develop. However, it has been observed in the experiments of Seifert and Pack [21] that the upstream boundary layer thickness has negligible effect on the flow parameters downstream of the hump. Thus the discrepancy in the turbulent state of the boundary layer upstream of the hump is not expected to have a major impact on the computed results downstream of the hump. 


\subsubsection{Streamwise distribution of Pressure Coefficient $C_{P}$}

The pressure coefficient $\mathrm{C}_{\mathrm{P}}$ is calculated as $\left(\mathrm{P}-\mathrm{P}_{\mathrm{ref}}\right) /\left(\frac{1}{2} \rho \mathrm{U}_{\infty}^{2}\right)$, where $\mathrm{P}_{\text {ref }}$ is the reference pressure, which is taken as the pressure at the inlet. Figure 5-4 below show the streamwise variation of the pressure coefficient obtained using WMLES compared against experiment as well as a case that not did employ the wall model on the same grid configuration.

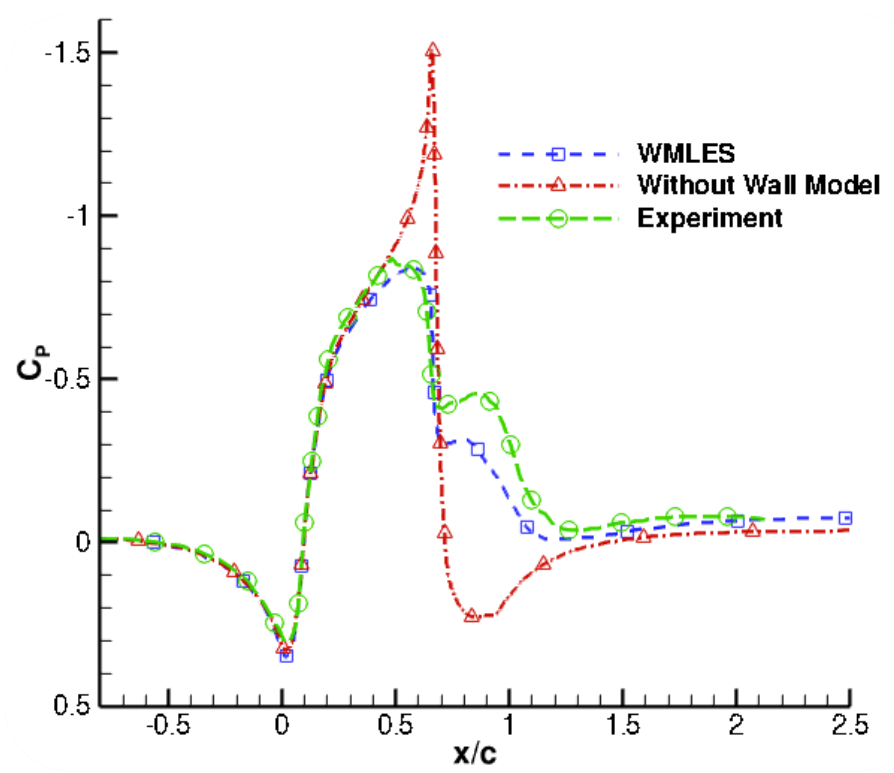

Figure 5-4: Variation of $C_{P}$ for WMLES on grid 792x128x64

It can be seen that the flow accelerates as it moves up the hump indicated by a decrease in $C_{p}$ to a value of -0.85 at about $\mathrm{x} / \mathrm{c}=0.5$. At around $\mathrm{x} / \mathrm{c}=0.65$ an increase in the value of $\mathrm{C}_{\mathrm{p}}$ can be seen indicating the adverse pressure gradient in the region. This adverse pressure gradient leads to flow separation. The WMLES is able to predict $C_{P}$ accurately until the flow reaches separation. There is a small region of favorable pressure gradient in the experimental data after the separation point, which is under predicted by the WMLES. The actual model on which the experiment was performed consisted of a hump enclosed between two end plates. These end plates create a blockage effect, leading to slight acceleration of the fluid in the region after separation. This is 
what causes the second suction peak, and since the computational model did not account for the end plates, this suction peak was not predicted by WMLES. Previous works on the same problem have observed that whenever the blockage was accounted for by altering the contour of the top

wall, this under prediction was not observed. [25, 27, 28, 44, 46, 50] A region of adverse pressure gradient follows this, which is smaller in magnitude compared to the experiment. Good agreement can be seen with the experiment following reattachment which occurs around $\mathrm{x} / \mathrm{c}=1.11$. However, the variation of $C_{P}$ without the wall model is very different from experiments from the time the flow reaches the separation point.

\subsubsection{Velocity Profiles}

Figure 5-5 shows the mean velocity profiles in the streamwise direction obtained from the simulation compared against the experimental values at locations just before separation to downstream of reattachment. The separation occurs at approximately $\mathrm{x} / \mathrm{c}=0.67$ and the experimental reattachment point is at $\mathrm{x} / \mathrm{c}=1.11$. 

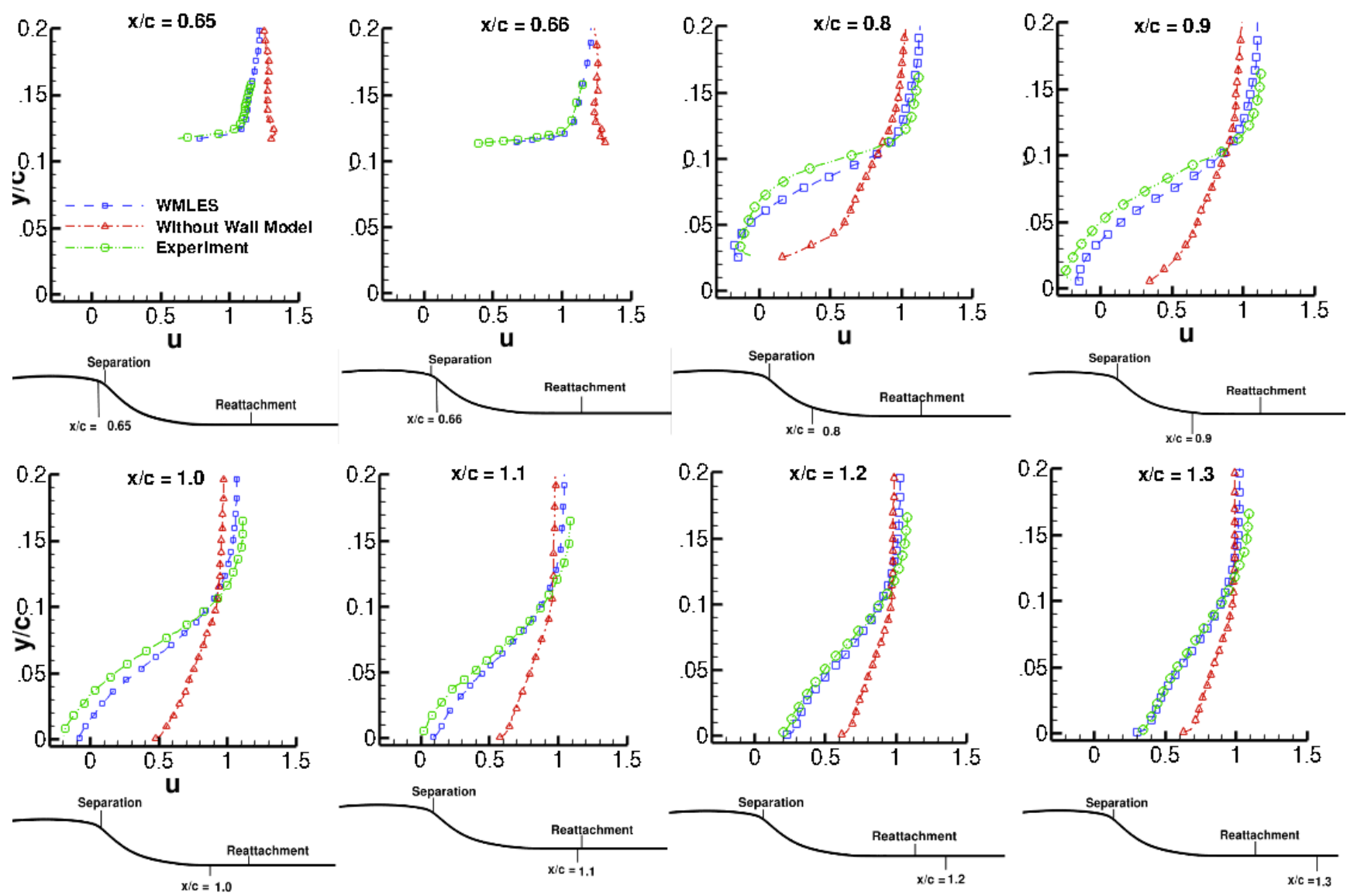

Figure 5-5: Mean velocity profiles from WMLES on grid 792x128x64

At locations 0.65 and 0.66 , which are slightly upstream to the separation region, WMLES accurately predicts the experimental profile. Within the recirculation region, at locations 0.8 and 0.9, the WMLES compares fairly well with the experiment, with WMLES profile exhibiting lower negative value indicating a possible earlier reattachment in comparison to the experiment. This could be due to the under prediction of the adverse pressure gradient in this region that occurs due to the blockage effect, which is unaccounted for in the simulation. Around the reattachment location, which is 1.11 as per the experiment, the WMLES again almost exactly reproduces the experimental profiles (locations 1.1 - 1.3). In contrast, the predictions of mean velocity profile without the use of the wall model show large discrepancies with the experimental profiles. The 
flow seems to accelerate quicker in the convex portion of the hump indicated by the higher velocities at locations 0.65 and 0.66 . There is no separation of the boundary layer observed following this, possibly indicating that the boundary layer remains laminar.

The contour plots of streamwise mean velocity in figure 5-6 below shows the streamlines forming a separation bubble in case of WMLES. However in the case without wall model, as shown in figure 5-7 no such separated region is seen.

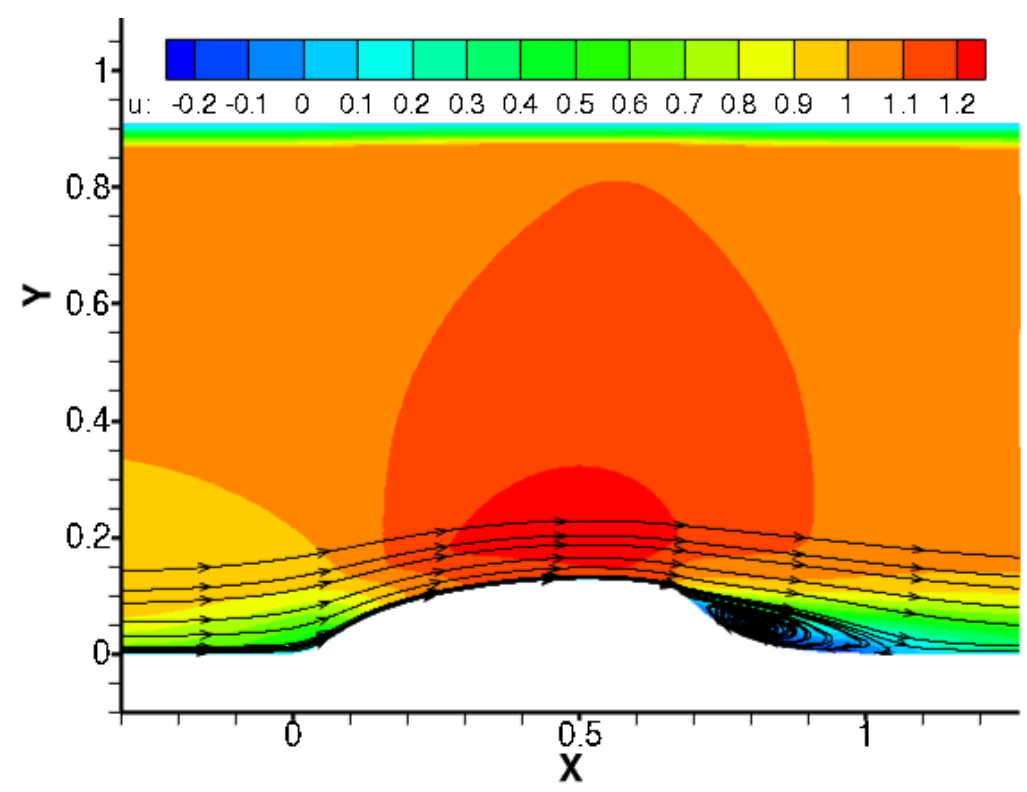

Figure 5-6: Contour of mean u-velocity with streamlines from WMLES 


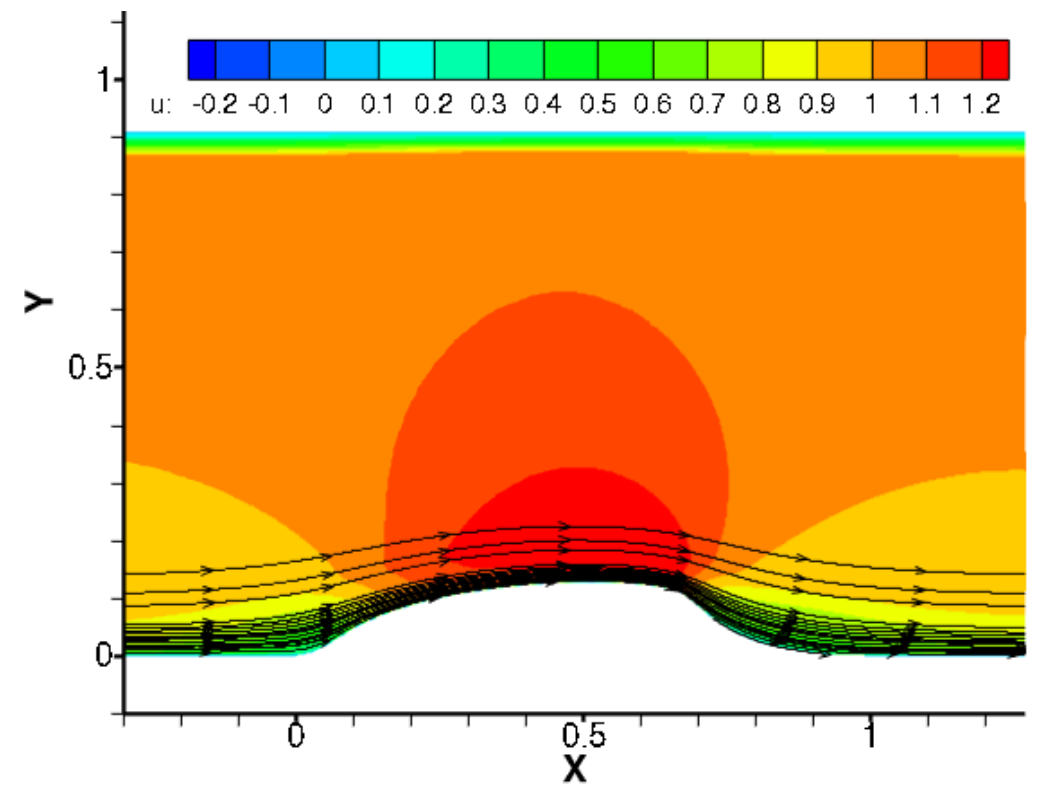

Figure 5-7: Contour of mean u-velocity with streamlines when wall model not used

\subsubsection{Turbulent parameters}

It is clear from the contours shown in the previous section that the flow does not separate when the wall model is not used. Figure 5-8 below shows the contour of turbulent $\overline{u^{\prime} u^{\prime}}$ as obtained from the WMLES while figure 5-9 is the case that did not employ wall model. 


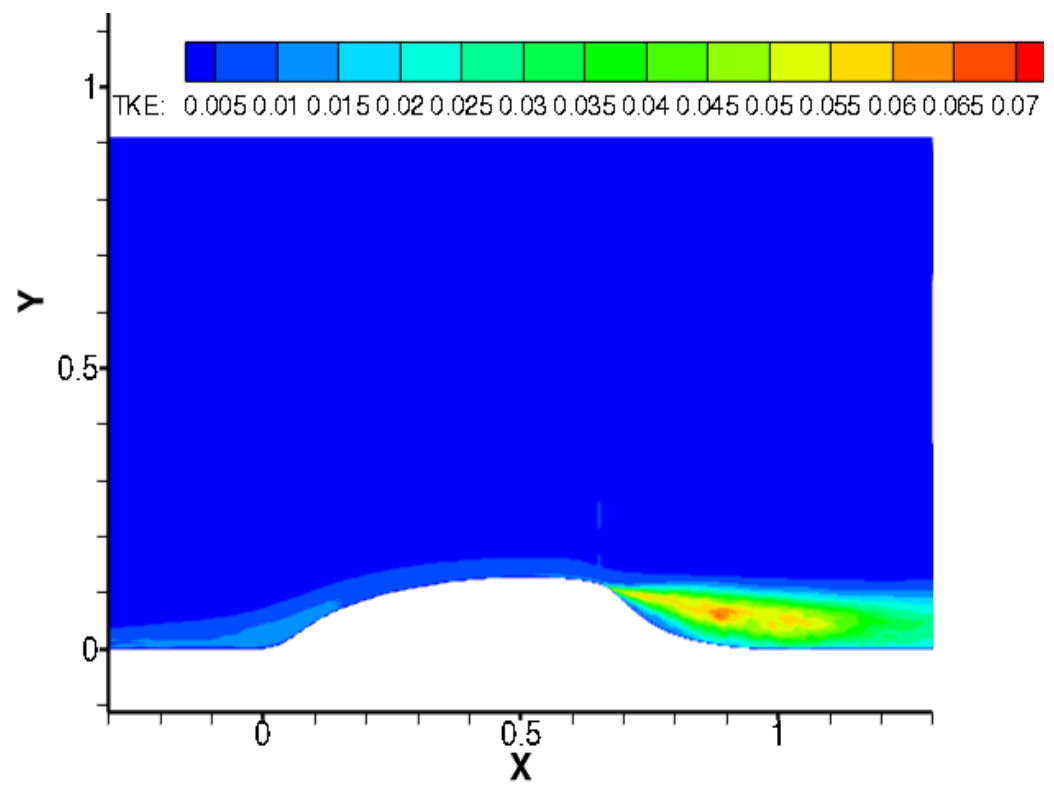

Figure 5-8: Contour of turbulent TKE from WMLES

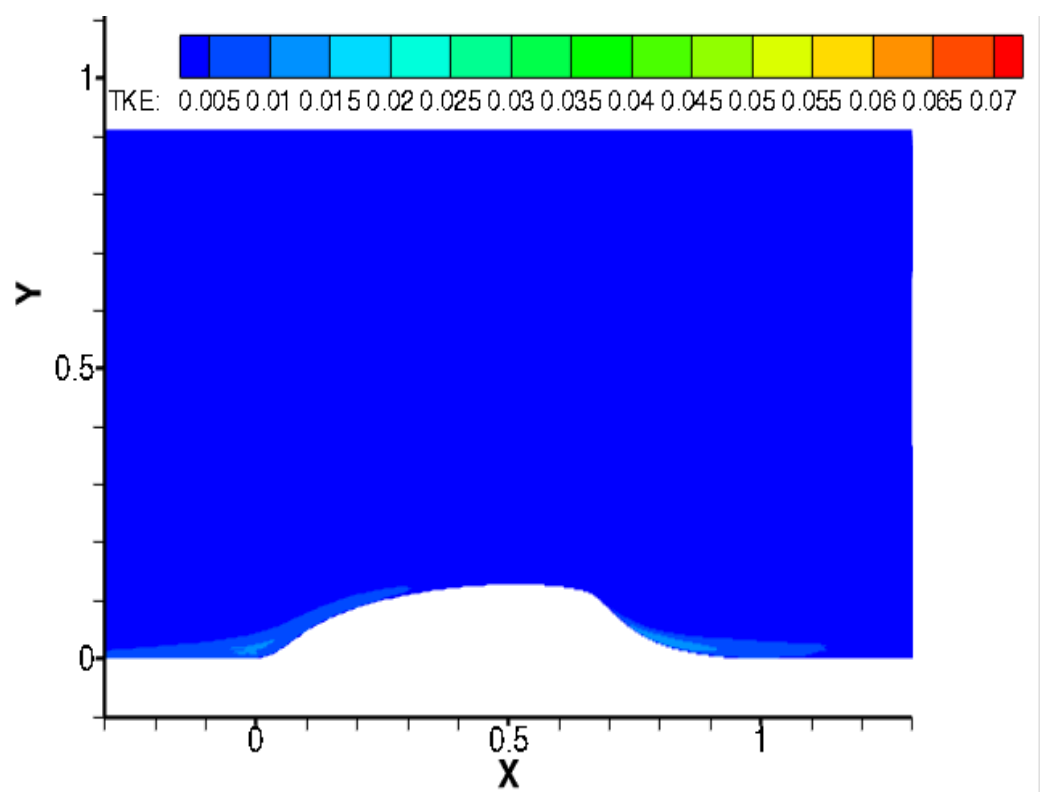

Figure 5-9: Contour of turbulent TKE without use of wall model

On comparison, it can be observed that the level of turbulence in the approaching boundary layer is lower in the case where wall model is not used in comparison to the WMLES. In both cases, the turbulence level decreases as the flow accelerates over the hump, though this leads to the flow not separating at all in the case where wall modeled is not used unlike WMLES 

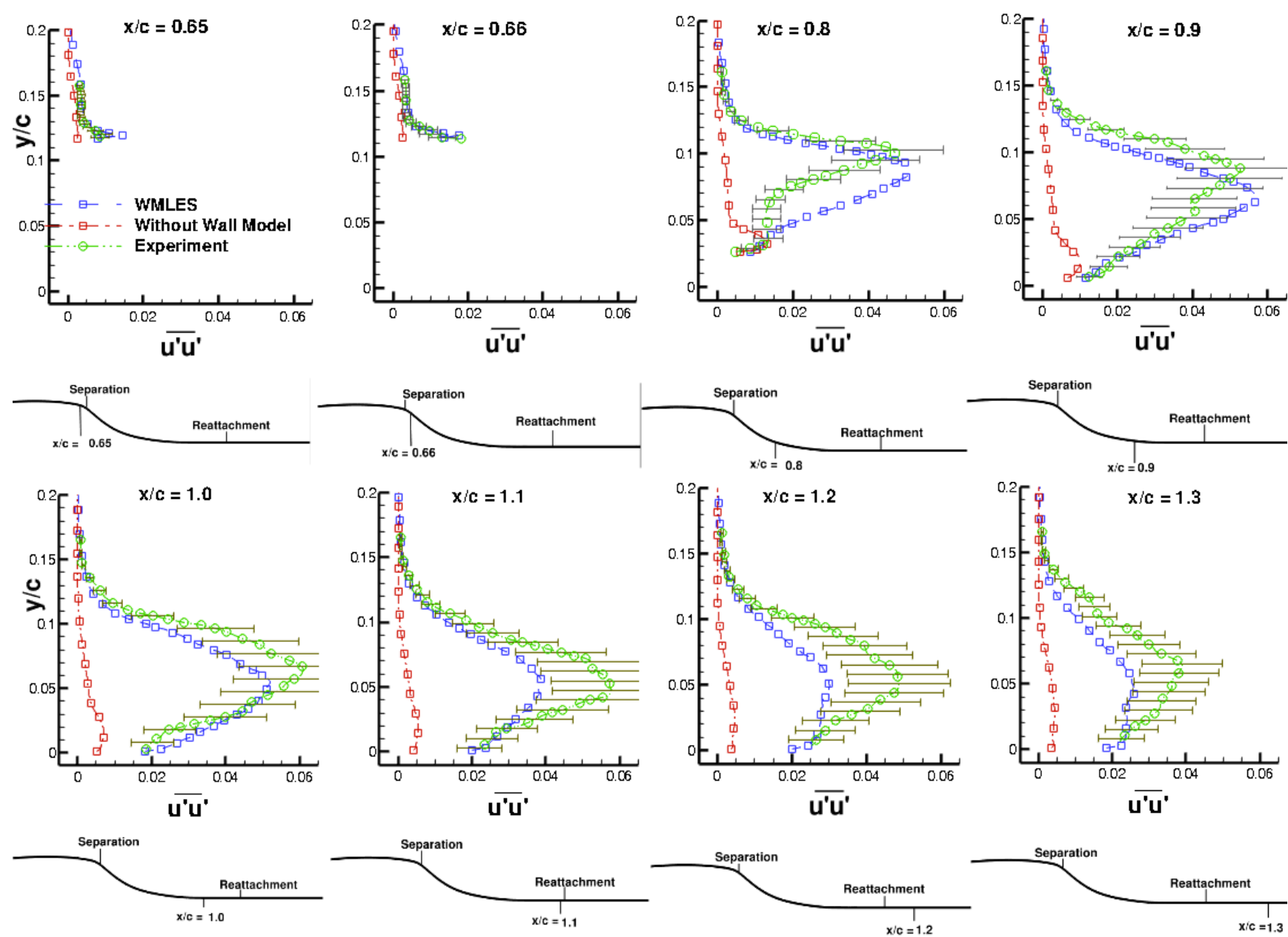

Figure 5-10: Profile of $\overline{u^{\prime} u^{\prime}}$ from WMLES on grid 792x128x64

Figure 5-10 and 5-11 show the profiles of turbulent normal stresses, $\overline{u^{\prime} u^{\prime}}$ and $\overline{v^{\prime} v^{\prime}}$ respectively predicted by the WMLES compared to experimental measurements. As mentioned in chapter 2, the experimental measurements had a maximum uncertainty of $14 \%$ for $\mathrm{u}_{\mathrm{rms}}$ and $\mathrm{v}_{\mathrm{rms}}$. Based on this the error bars are plotted in the figures. Both $\overline{u^{\prime} u^{\prime}}$ and $\overline{v^{\prime} v^{\prime}}$ are over predicted within the boundary layer at locations 0.65 and 0.66 that are slightly upstream of the separation point. This same trend (over prediction of peak values in boundary layer) is also seen in other past computational studies $[36,44]$. After separation at $\mathrm{x} / \mathrm{c}=0.67$, the turbulent stresses are maximum in the separated shear layer which forms between the outer flow and the recirculation region. The prediction of peak normal stresses agree well with the experiments at $\mathrm{x} / \mathrm{c}=0.8$ and 0.9 in the case of $\overline{u^{\prime} u^{\prime}}$ while $\overline{v^{\prime} v^{\prime}}$ 
is over predicted at $\mathrm{x} / \mathrm{c}=0.8$. Following this region, there is sharp decay in WMLES. This is followed by an under prediction of the peak stresses near and after reattachment at $\mathrm{x} / \mathrm{c}=1.0,1.1$, 1.2, and 1.3, although they are still within the uncertainty band at $\mathrm{x} / \mathrm{c}=1.0$ in case of $\overline{u^{\prime} u^{\prime}}$ and at $\mathrm{x} / \mathrm{c}=1.0$ and 1.1 in case of $\overline{v^{\prime} v^{\prime}}$. While the peak normal stress increase up to $\mathrm{x} / \mathrm{c}=1.0$ in the experiments, the predicted stresses start to decay earlier after $\mathrm{x} / \mathrm{c}=0.9$.
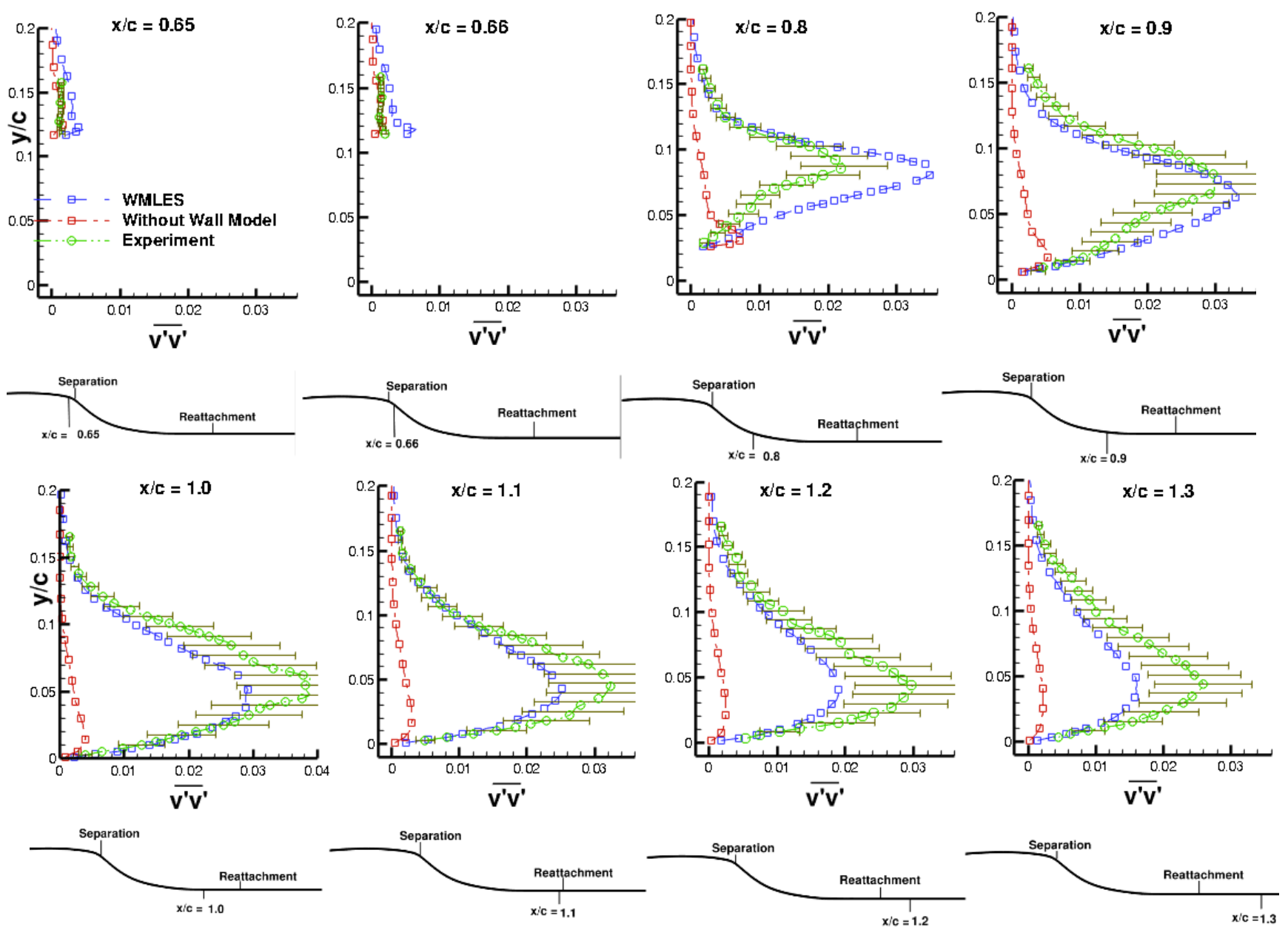

Figure 5-11: Profile of $\overline{v^{\prime} v^{\prime}}$ from WMLES on Grid 792x128x64

It is noted that in the absence of the wall model, the normal stresses are in somewhat better agreement with the experiments at $\mathrm{x} / \mathrm{c}=0.65$ and 0.66 before separation, but are grossly under predicted with completely different profile shapes after separation. The turbulent stress predictions 
together with the mean field predictions show clearly that in the absence of the wall model, there is no separation on the concave surface.

Figure 5-12 shows the turbulent shear stress predicted by the WMLES compared with the experimental data. The uncertainties from the experiments are also indicated. The shear stress exhibits the same trend as that for $\overline{u^{\prime} u^{\prime}}$ and $\overline{v^{\prime} v^{\prime}}$. At locations 0.65 and 0.66 there is slight over prediction of $\overline{u^{\prime} v^{\prime}}$ by WMLES in comparison to experiment. The agreement is fairly good at locations 0.8 and 0.9 , which are in the recirculation zone, followed by under-prediction at locations 1.0 through 1.3
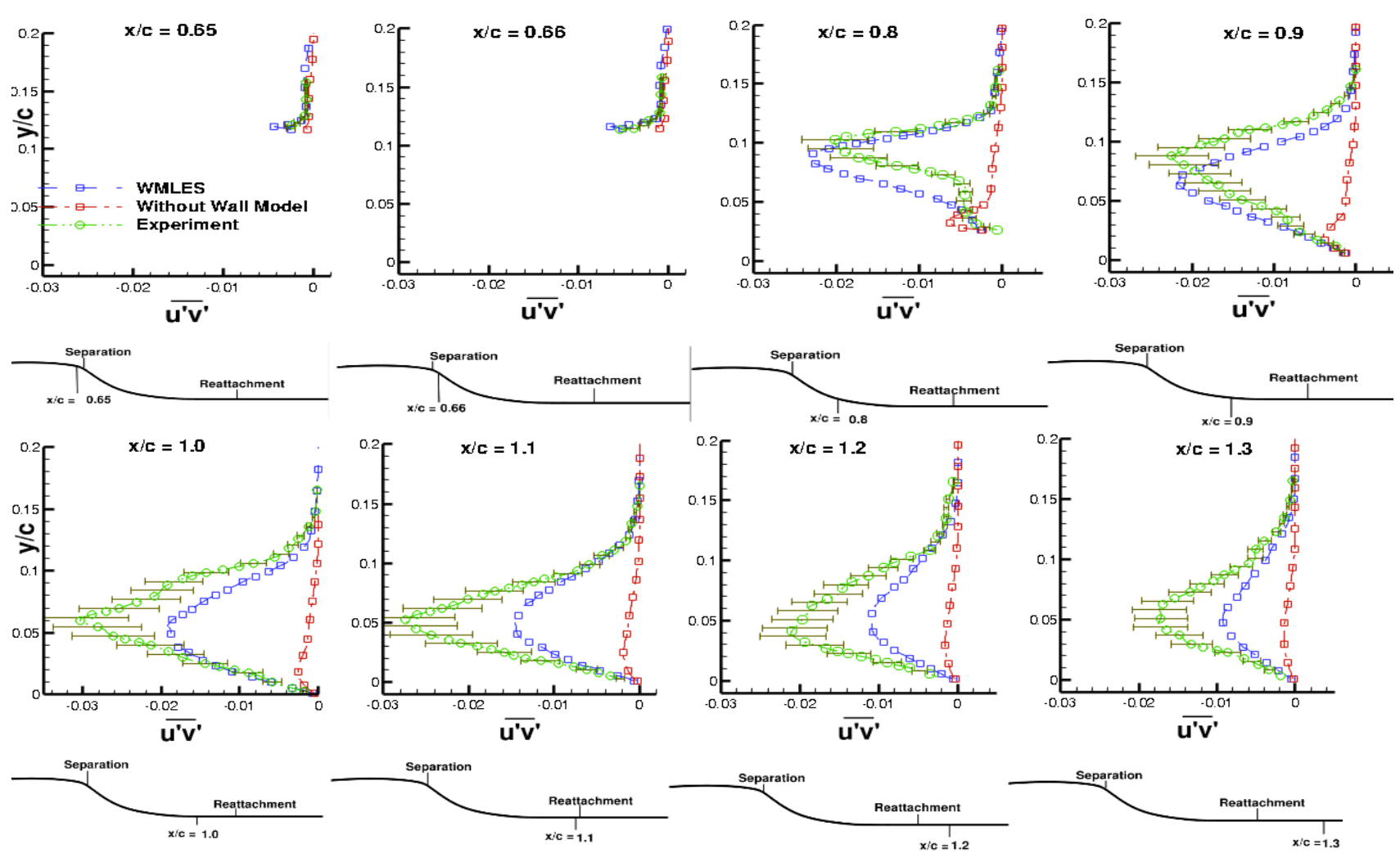

Figure 5-12: Profile of $\overline{u^{\prime} v^{\prime}}$ from WMLES on grid 792x128x64

To compare the profile of turbulent quantities with the observations from previous studies, a few points mentioned in chapter 3 are reiterated here. The trends shown by the turbulent quantities and coefficient of pressure as obtained from the WMLES are similar to the results obtained from the 
LES of Saric et al. as mentioned in chapter 3. [36] Their work, which employed an LES with the Smagorinsky model for modeling sub-grid scales on a collocated grid, shows the over prediction of turbulent quantities at locations 0.65 and 0.66 , fair agreement at locations 0.8 and 0.9 and under prediction for locations 1.0 through 1.3. Their simulation, like in the present study, did not account for the end plate blockage and consequently had a different pressure gradient than the experiments in the region $(0.75<\mathrm{x} / \mathrm{c}<1.2)$. The results obtained by Avdis et al. [50] at the same Reynolds number as the present work showed over prediction of the turbulent parameters at most locations, with their predictions agreeing best with experiments at location $\mathrm{x} / \mathrm{c}=1.1$. They used implicitly filtered LES applied on a collocated finite volume grid. The work of Morgan et al. [44] shows over prediction of turbulent parameters at locations 0.65 and fair agreement with experiment at location 0.8 , both consistent with the present WMLES. However, their results at locations 1.0 and 1.2 agree more closely with the experiment than the present predictions. Morgan et al. [44] used implicit LES (ILES) and fourth order spatial compact difference formulations and their simulations were performed at a reduce Reynolds number of $2.0 \times 10^{5}$. This could be the reason for the results agreeing more closely with the experiment. You et al. [46] provide another set of results using LES with the dynamic Smagorinksy model to calculate the sub-grid stresses on a staggered grid. The Reynolds stress obtained from their calculation agrees more closely with experiment at location 1.2 unlike the present WMLES. However their computational domain had made changes to the contour of the top wall to account for the end plate blockages.

The reason for the decreased accuracy in prediction of turbulent stresses towards the end of the recirculation region could also be due to a number of other factors viz., the discretization scheme, the grid resolution, the LES method employed or due to the grid configuration (staggered/collocated). The effect of grid resolution shall be presented later in the present section. 
It can be seen that Saric et al. used the same method as in the present LES which is an SOC scheme for spatial discretization on a collocated grid, and the results obtained by them is similar to the present work. Morgan et al. used higher order spatial discretization schemes and performed the calculation at a lower Reynolds number. You et al. used similar parameters for their calculation as the present work with the exception that they employed a staggered grid instead of a collocated grid.

It should be noted that the WMLES is able to predict the mean velocity experimental data very well while the calculations performed without using a wall model for the same grid configuration fails to predict the flow features at all data locations. With the exception of couple of locations, the wall model is also able to reproduce the turbulent data fairly well.

\subsubsection{Skin friction and Reattachment Length}

Figure 5-13 shows the streamwise skin friction coefficient as obtained from the present WMLES. The experimental profile as well as the profile obtained when no wall model is used is shown as well in the figure for comparison. 


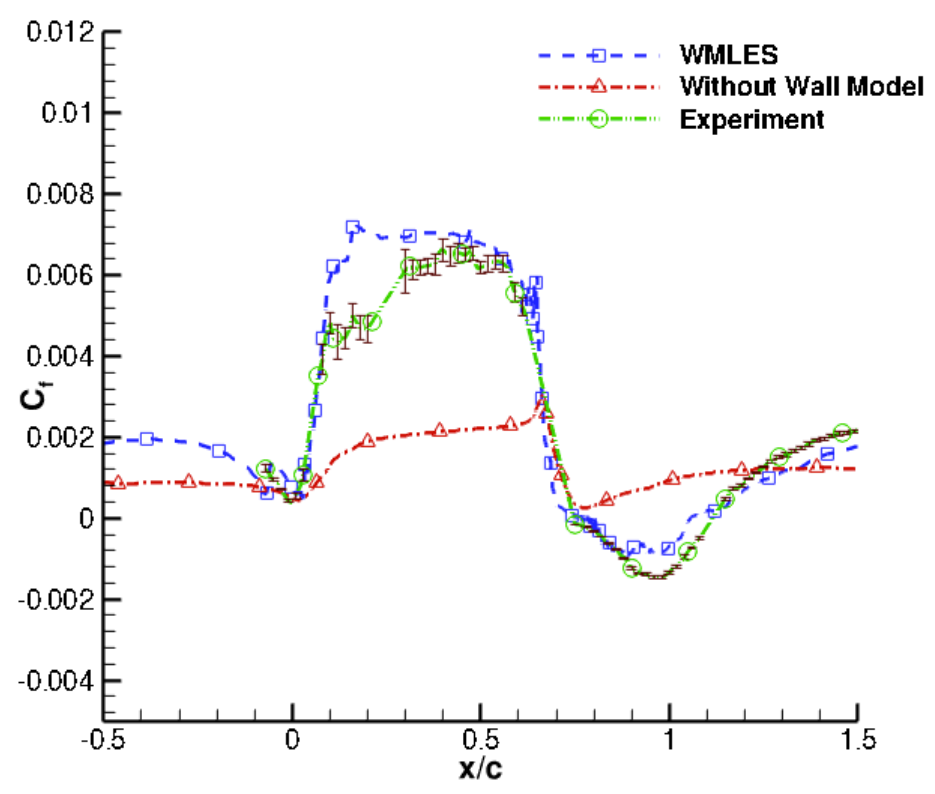

Figure 5-13: $C_{f}$ from WMLES on Grid 792x128x64

It is observed that the simulation agrees fairly with the experiment, except during the initial part of the hump, where the predicted skin friction is higher than the values obtained experimentally. It was seen earlier that the boundary layer obtained from WMLES showed higher level of turbulence than experiment at locations 0.65 and 0.66 . This means that the computed level of turbulence is higher than the experiment for the first half of the hump, and this might be the reason for a higher value of skin friction coefficient in this region. Besides, the predicted value is not very much offset from the experimental errors bars shown in the region. From the above figure, the reattachment length is obtained as 1.08 , while the experimental reattachment is at $1.11 \pm$ 0.003. The accuracy of the reattachment length prediction is comparable, if not better, to those from the LES performed by Saric et al., Morgan et al. and Moin et al. who got the values as 1.114, 1.127 and 1.09 , respectively. 


\subsection{WMLES grid refinement study}

The results presented in the previous section were found to agree well with the experiment in case of streamwise pressure coefficient, skin friction, and mean velocity profiles. However, this was not the case with turbulent quantities, where under prediction of peak magnitudes were observed at a number of locations. It can be argued that increasing the grid resolution can bring about additional improvement in the accuracy of the results. From figure 4-2 shown earlier, it was seen that in the value of $\Delta \mathrm{x}^{+}$was comparable to $\Delta \mathrm{y}^{+}$in the region $0.65<\mathrm{x} / \mathrm{c}<1.3$, with a maximum value of 200 in the first half of the hump. In case of $\Delta z^{+}$, a uniform distribution of 64 cells in $\mathrm{z}$ direction yielded a value of approximately 50 for most of the recirculation region.

The LES approach presented in this study employs an implicit filtering technique where the computational grid size acts as the filter width to demarcate the resolved and subgrid scales. In such a case, an improvement in grid resolution is bound to improve the accuracy of the simulation, as the more refined the grid is, the closer the calculation is to a direct numerical simulation (DNS). Under such a scenario, it is necessary to estimate the size of the grid beyond which a further refinement does not justify the increase in computational cost in terms of the observed increase in accuracy.

This section presents the results from a calculation performed to understand the effect of improving the grid resolution on the overall accuracy of the simulation. For this purpose, the number of grid points in all the three co-ordinate directions was increased by a factor of 1.5 . It is noted that the distance of the first off-wall grid point was kept the same as earlier calculations so as to keep the $\mathrm{y}^{+}$value between $20-40$ for the application of a wall model. Beyond the first grid point the resolution was improved by the factor mentioned above. The refined grid had 1188, 
192 and 96 cells, respectively in the $\mathrm{x}, \mathrm{y}$ and z-direction giving an overall size of approximately 22 million cells. All the other parameters of the simulation were kept same.

Figures 5-14 and 5-15 and show streamwise variation of the coefficient of pressure and skin friction, respectively, obtained from the new simulation, compared against the WMLES results presented earlier as well as the experimental data.

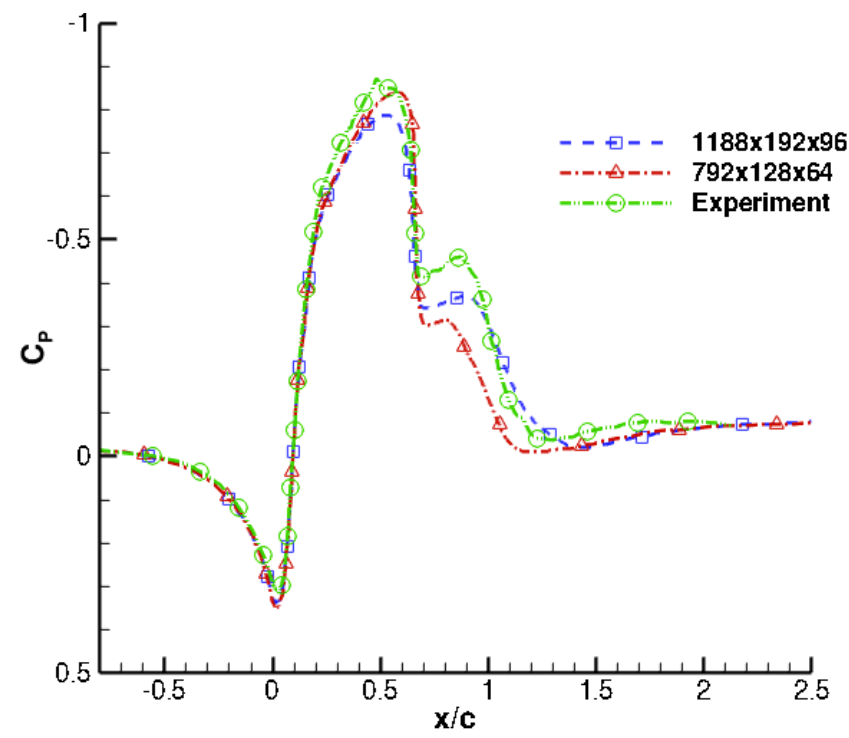

Figure 5-14: $C_{P}$ WMLES on grid 1188x192x96

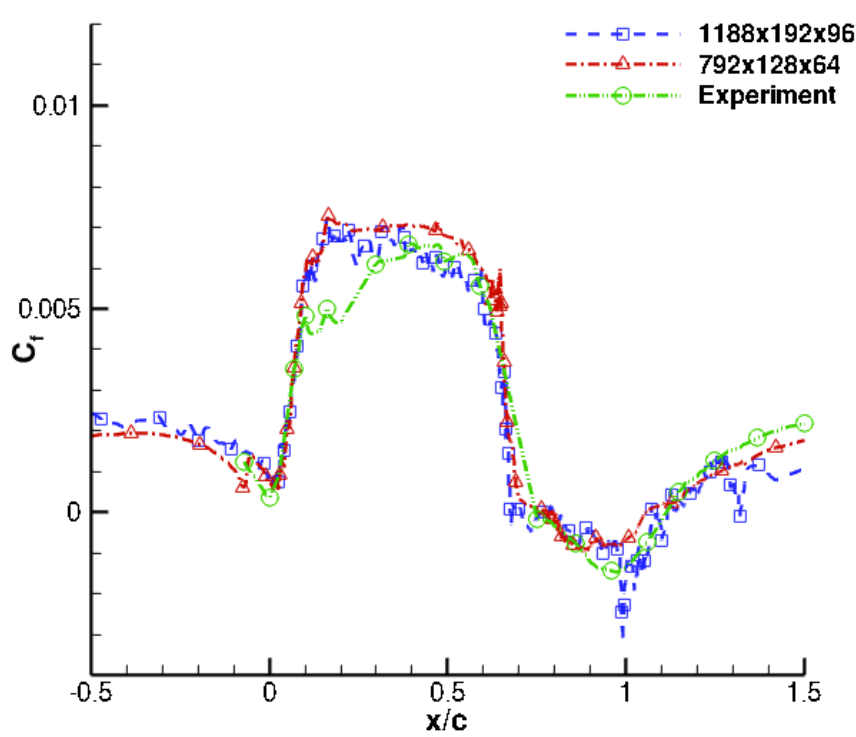

Figure 5-15: $\mathrm{C}_{\mathrm{f}}$ from WMLES on grid $1188 \times 192 \times 96$

The pressure coefficient shows a more accurate prediction of pressure recovery in the separated region $(\mathrm{x} / \mathrm{c} \sim 0.8)$ with the use of the refined grid, with the beginning of recovery predicted closer to the reattachment location than on the coarser grid. The plot of skin friction shows a negative peak in the region just upstream of the reattachment point with the refined grid, which was not observed on the coarser grid. The reattachment location is obtained as $\mathrm{x} / \mathrm{c}=1.12$ in comparison to $\mathrm{x} / \mathrm{c}=1.08$ obtained earlier. 
Figure 5-16 - 5-18 shows the profiles of Reynolds stresses and figure 5-19 shows the profile of mean streamwise velocities obtained from the simulation performed on the refined grid compared against the WMLES results presented earlier and the experimental data.
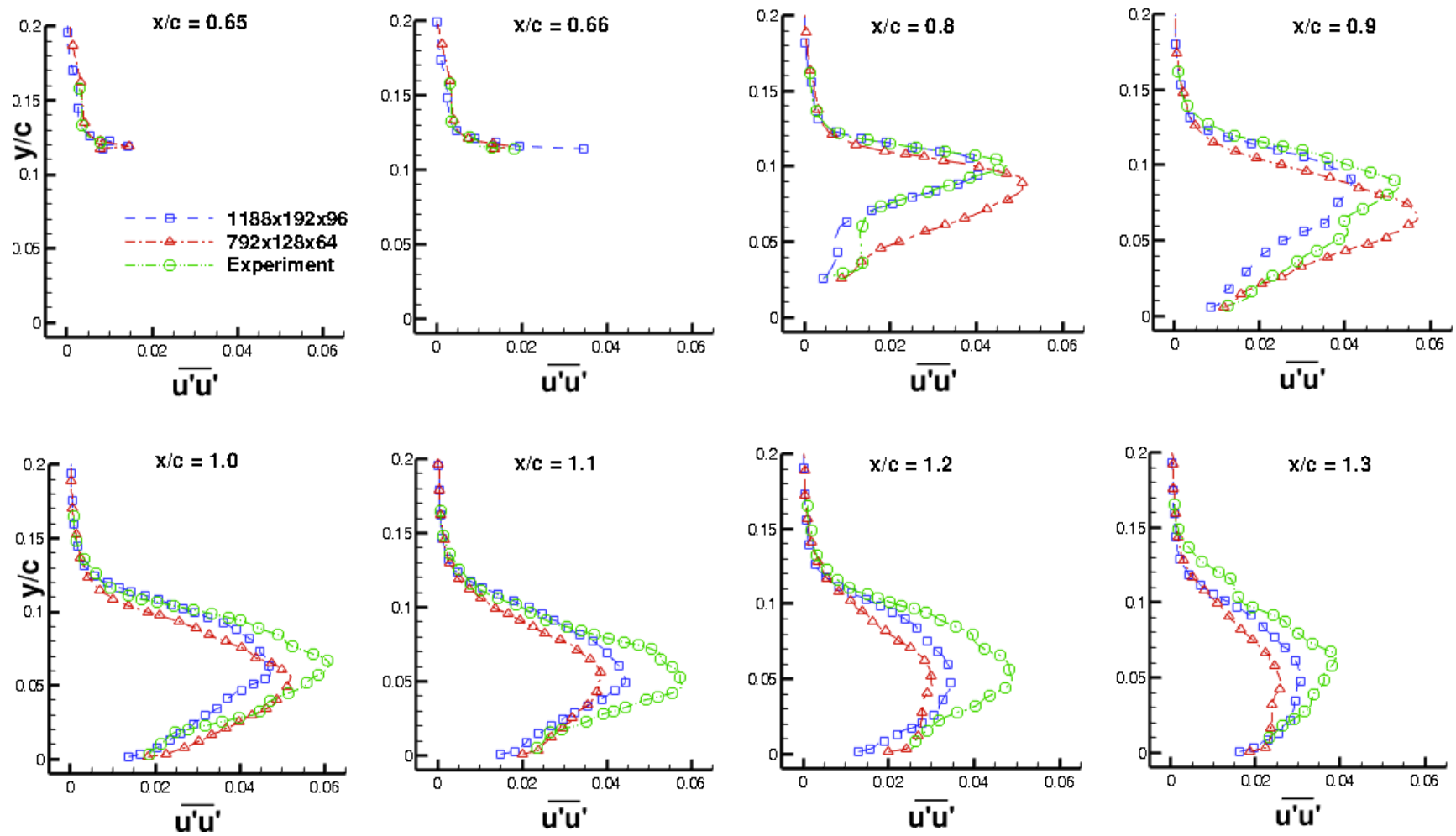

Figure 5-16: Profile of $\overline{u^{\prime} u^{\prime}}$ from WMLES on grid 1188x192x96
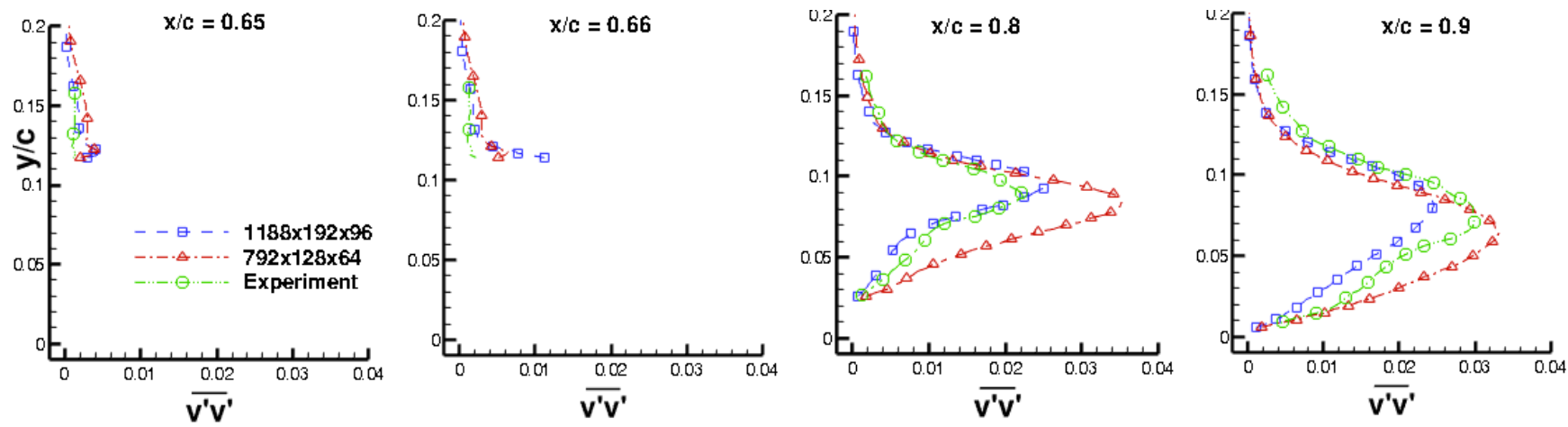

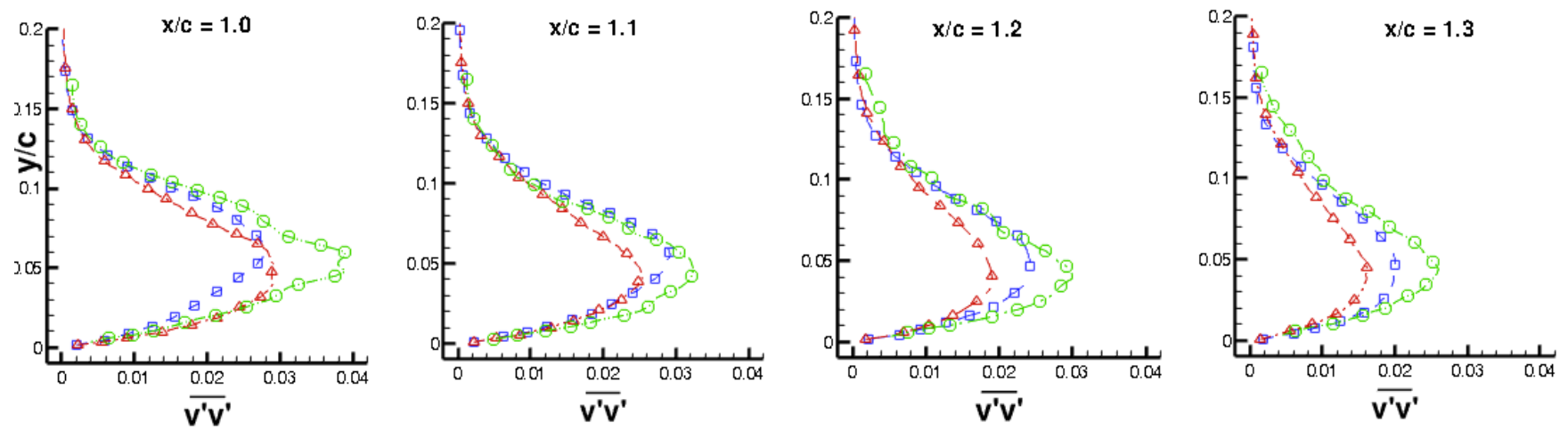

Figure 5-17: Profile of $\overline{v^{\prime} v^{\prime}}$ from WMLES on grid 1188x192x96
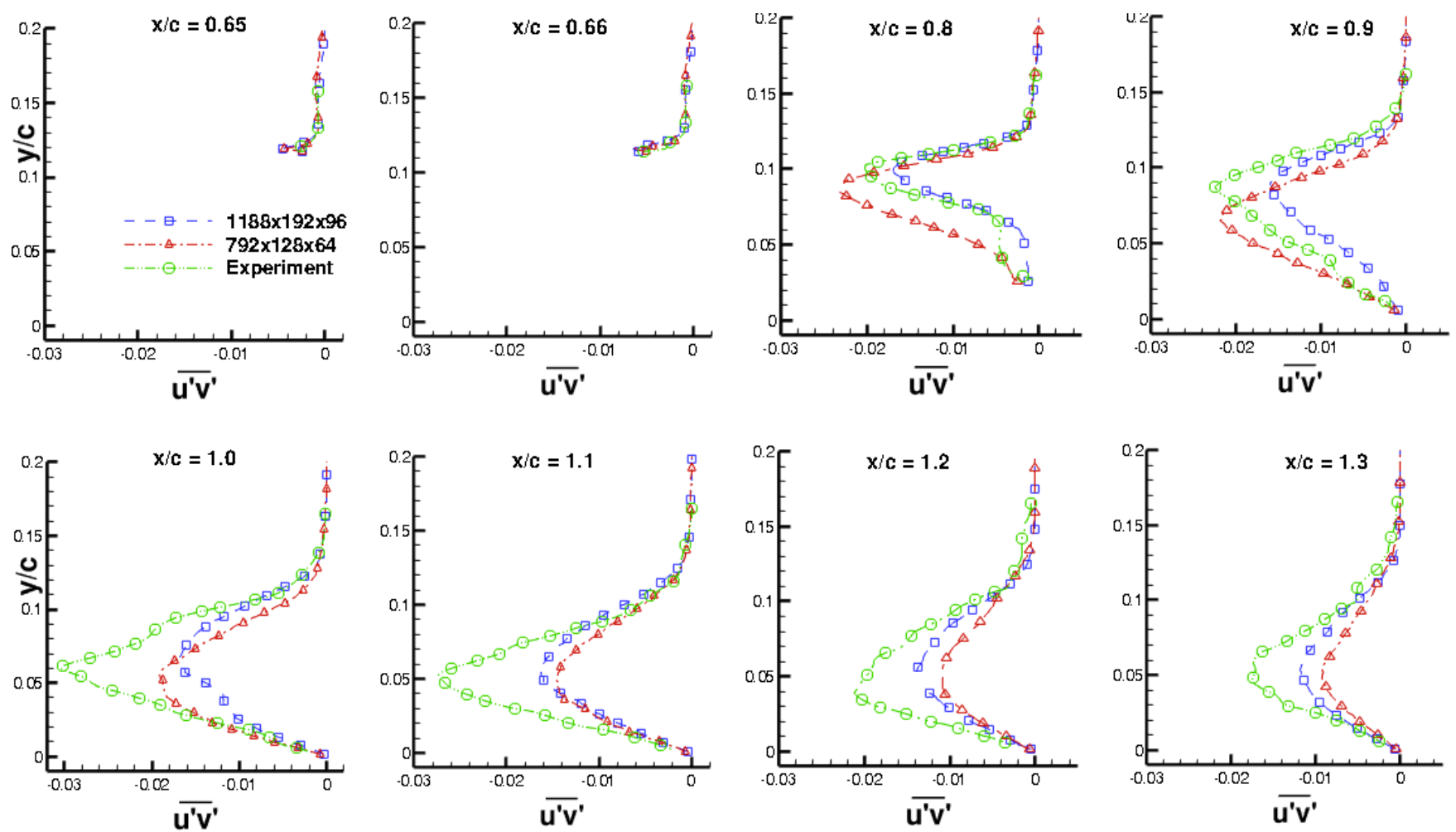

Figure 5-18: Profile of $\overline{u^{\prime} v^{\prime}}$ from WMLES on grid 1188x192x96
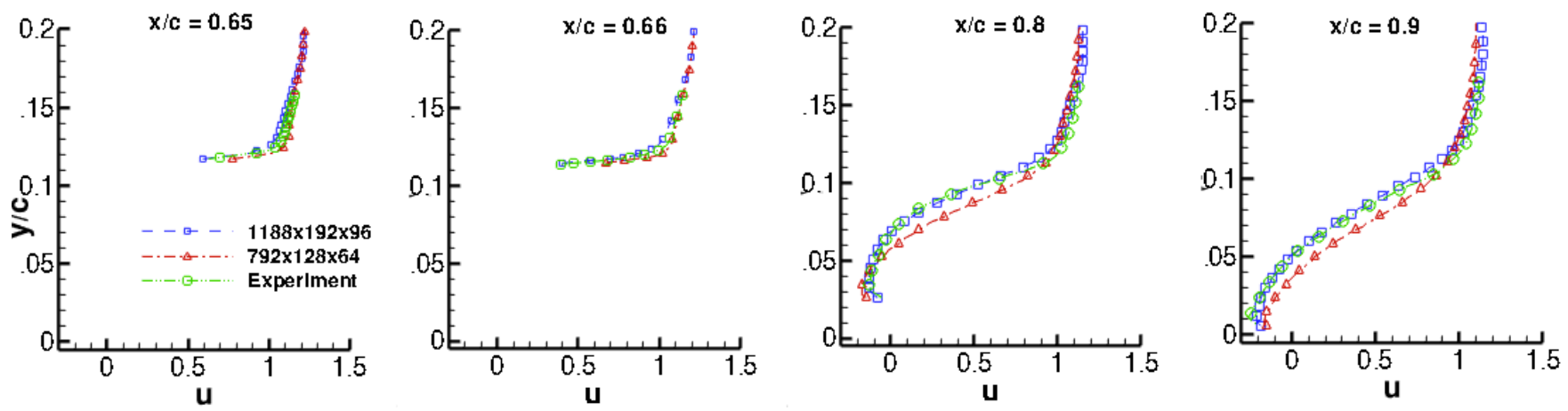

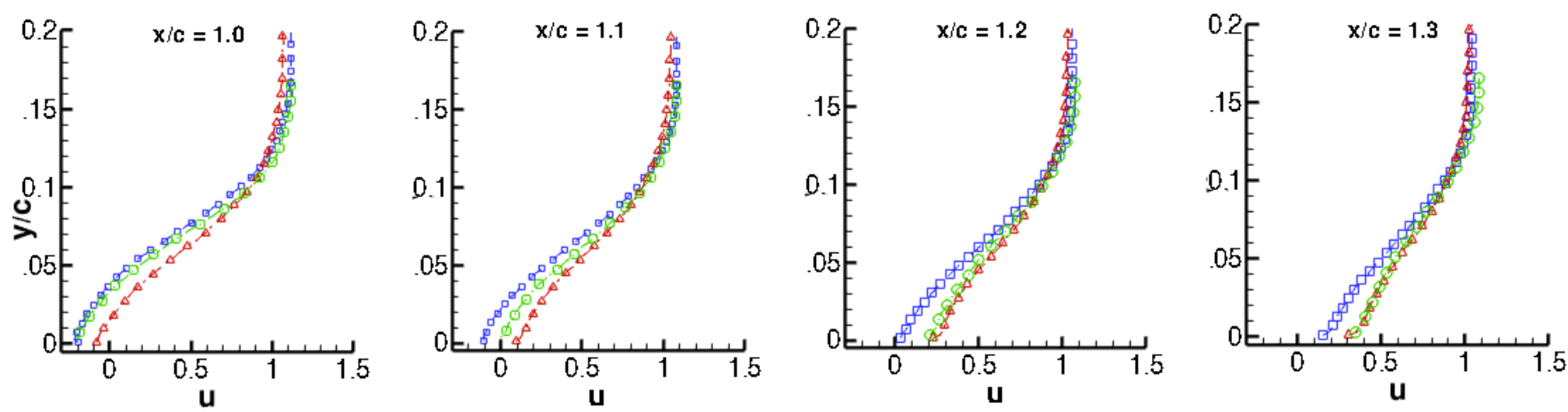

Figure 5-19: Profile of mean streamwise velocity from WMLES on grid 1188x192x96

The profile of $\overline{v^{\prime} v^{\prime}}$ moves towards the experimental profile in the region after the reattachment location $(\sim \mathrm{x} / \mathrm{c}=1.0)$. However, in case of $\overline{u^{\prime} u^{\prime}}$ and $\overline{u^{\prime} v^{\prime}}$ the improvement is marginal. All the Reynolds stress profiles at location $\mathrm{x} / \mathrm{c}=0.8$ show considerable improvement, while they continue to exhibit discrepancies with the experiment at other locations. In case of mean velocity profiles, the results from the refined grid show better agreement with the experiment up till the reattachment point, beyond which discrepancies are observed.

It can be concluded from this study that the improvement in grid resolution has some impact on the simulation results as expected. However, whether the increase in accuracy is significant enough to justify the 3.4 times increase in the grid size or computational cost is debatable. This conclusion is validated by other studies in the literature, which have used an equivalent LES grid similar to the coarser grid used in this study barring the resolution in the wall normal direction, which is typically more than used in this study.

In most previous work with WRLES, 64 cells or more are used in the spanwise z-direction. It was observed that reducing the number of cells to 32 cells in the z- direction quickly led to an unstable calculation that diverged. This implies that the proper resolution of the near wall 
structures requires a spanwise grid of certain minimum resolution. Since the primary objective behind wall modeling is to reduce the extreme grid resolution requirement that accompanies a wall resolved LES of a high Reynolds number flow, the effect of reducing the grid resolution in the spanwise z-direction is investigated. Hence results from a WMLES calculation performed on a grid of size $792 \times 128 \times 32$, which is half the size of the grid used earlier, is presented. It can be inferred from figure 4-2 that for this grid configuration, a uniform distribution of 32 cells in $\mathrm{z}$ direction would yield a maximum value of approximately 180 for $\Delta \mathrm{z}^{+}$, and stays around the value of 100 for most of the recirculation region.

Figure 5-20 and figure 5-21 show the comparison of calculated streamwise pressure coefficient and skin friction, respectively, between the original grid and the z-coarsened grid.

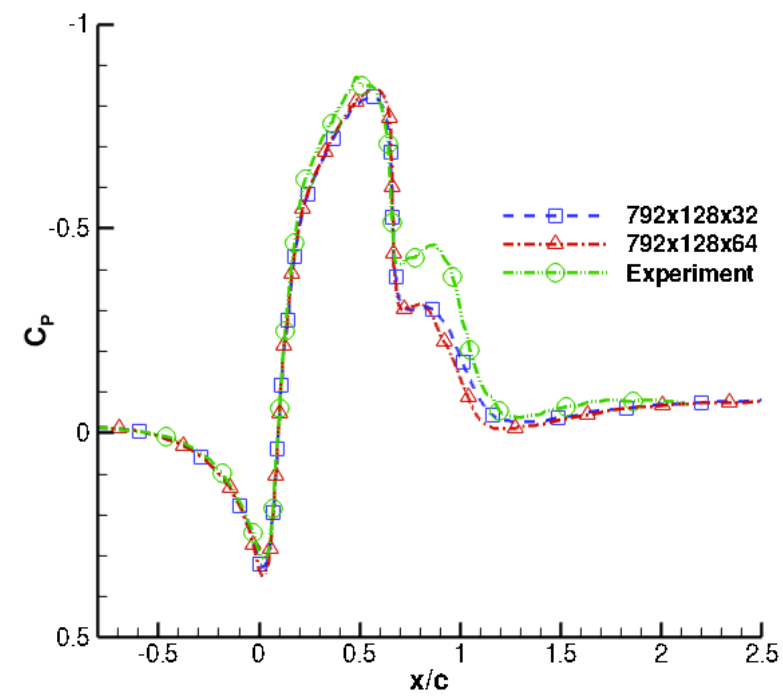

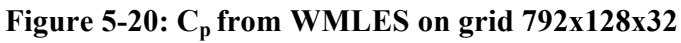

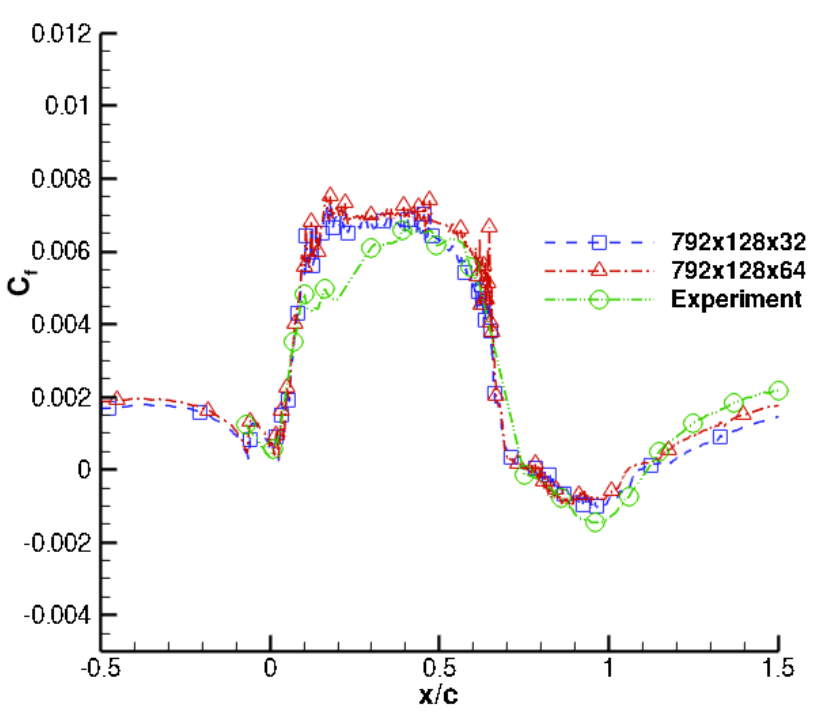

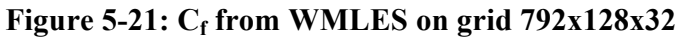

It can be seen that the increased resolution in z-direction does not have a significant influence on the prediction of skin friction coefficient and pressure coefficient as compared to using the original grid. In case of the pressure coefficient, there still exists a difference between the experiment and simulation in the prediction of the suction peak around $\mathrm{x} / \mathrm{c}=0.65$, due to the end plate blockage 
effect. In case of the skin friction coefficient there is almost negligible difference between the predictions with the new coarser grid slightly over predicting the value of $\mathrm{C}_{\mathrm{f}}$ over the experiment in the first half of the hump. The reattachment location obtained on the finer grid is 1.1 versus 1.08 on the original grid. In both cases, the difference between the experimental and the predicted reattachment length is less than $7 \%$.

Figure 5-22 shows the comparison between the streamwise mean velocity obtained from the coarse grid and the results from the original grid.
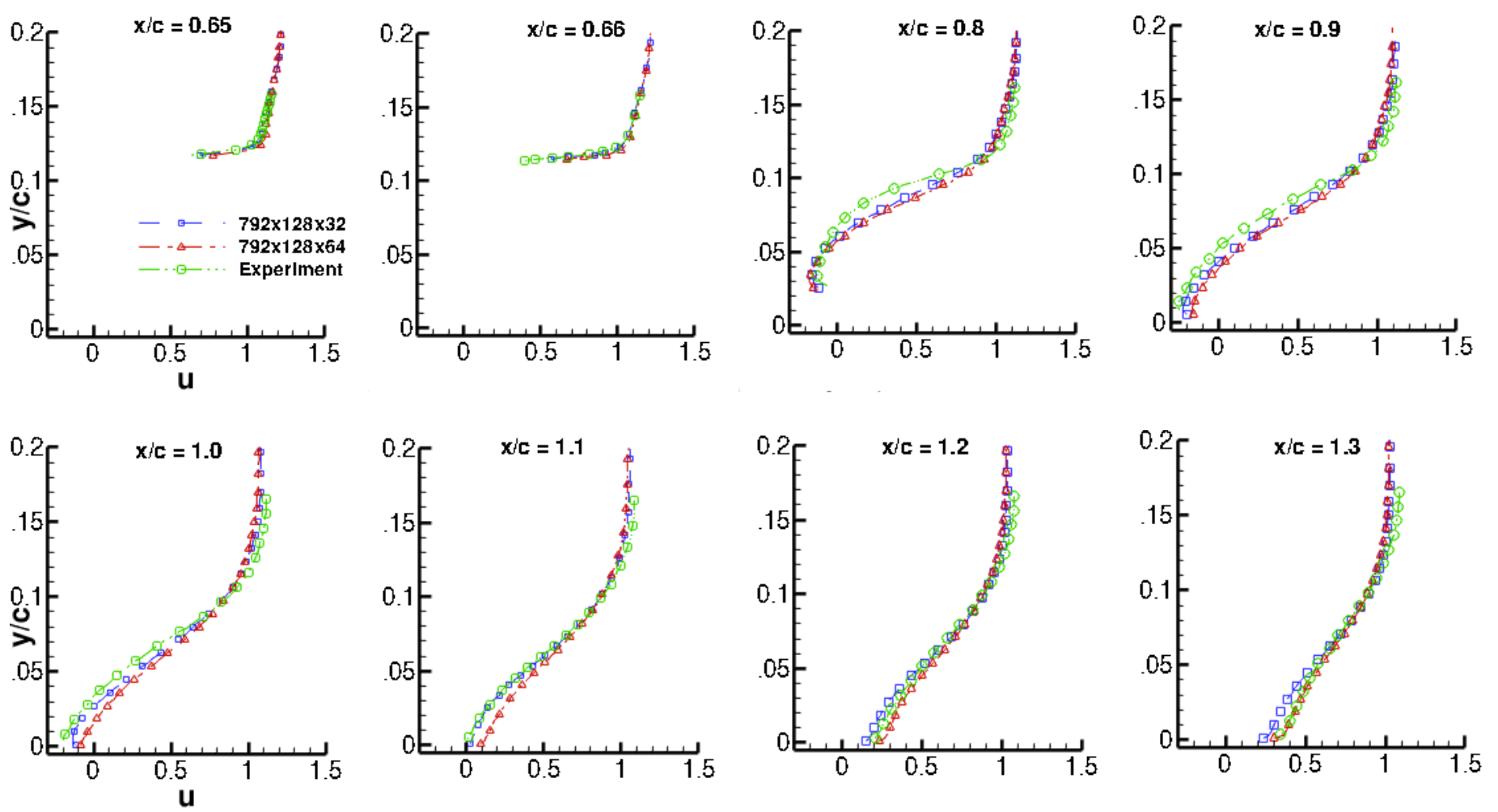

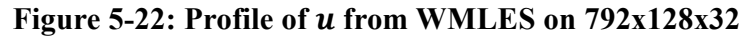

It can be seen the profiles are almost identical in most locations. Near the reattachment location, i.e., $\mathrm{x} / \mathrm{c}=1.0$ and 1.1 , the new velocity profile is closer to the experimental profile implying that the velocity profile from the WMELS on $792 \times 128 \times 32$ is not recovering as fast as 
the velocity profile from $792 \times 128 \times 64$. However, at location 1.1 the agreement of both profiles with the experiment is similar, with the profile from $792 \times 128 \times 64$ agreeing slightly better at location $\mathrm{x} / \mathrm{c}=1.3$

Figure 5-23 - 5-25 show the comparison between the turbulent quantities obtained from the refined grid and the original grid.
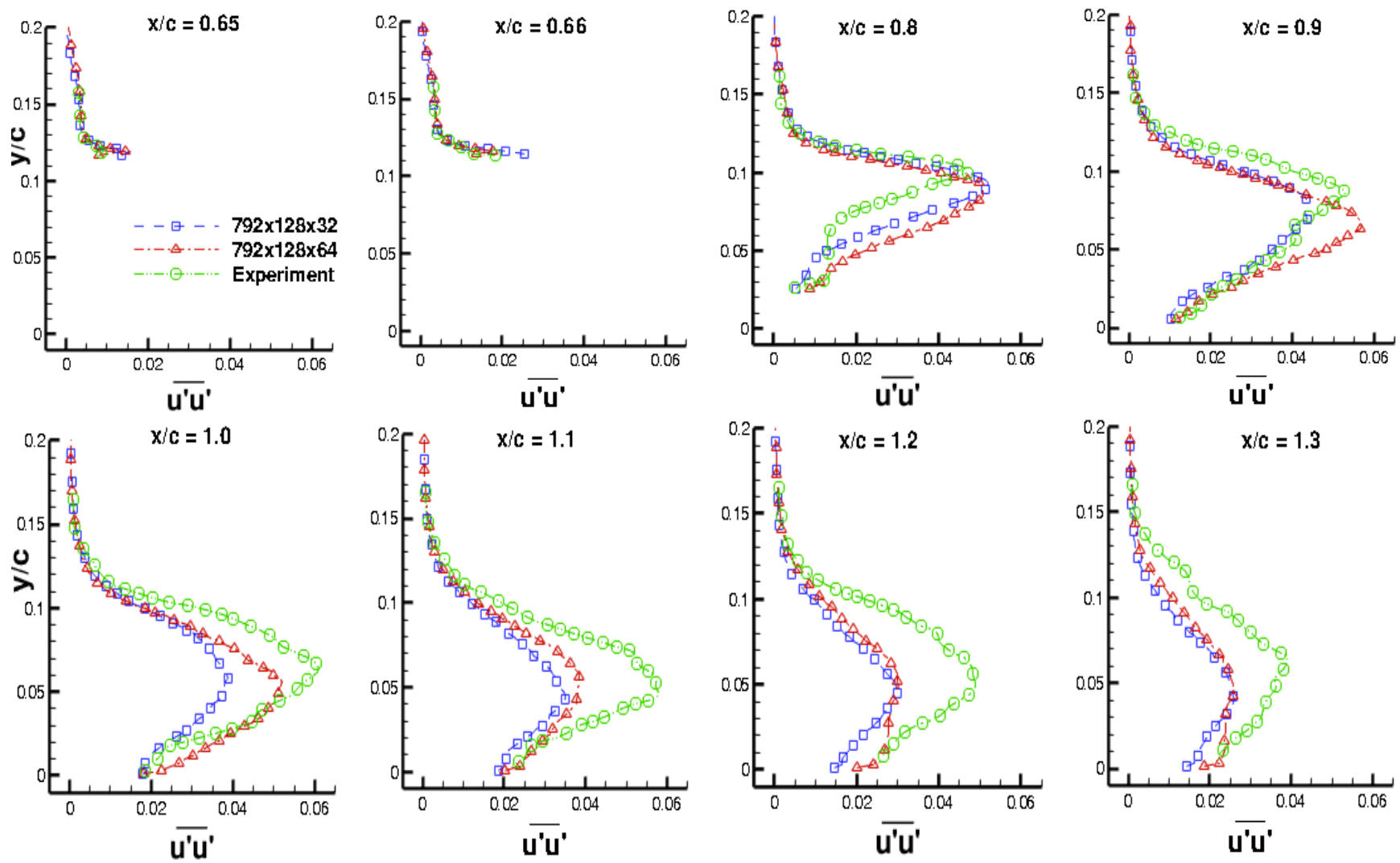

Figure 5-23: Profile of $\overline{u^{\prime} u^{\prime}}$ from WMLES on $792 \times 128 \times 32$ 

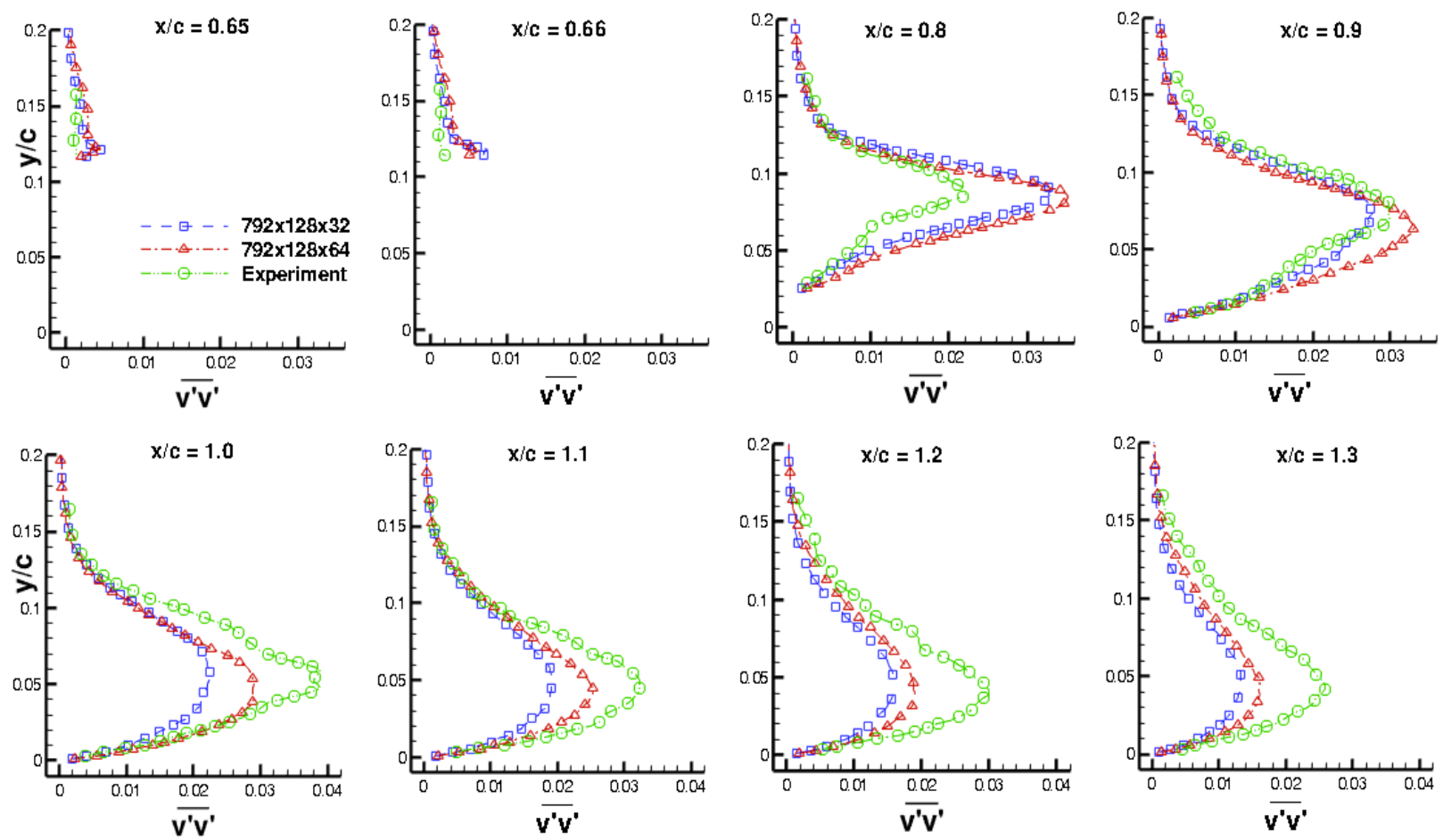

Figure 5-24: Profile of $\overline{v^{\prime} v^{\prime}}$ from WMLES on 792x128x32
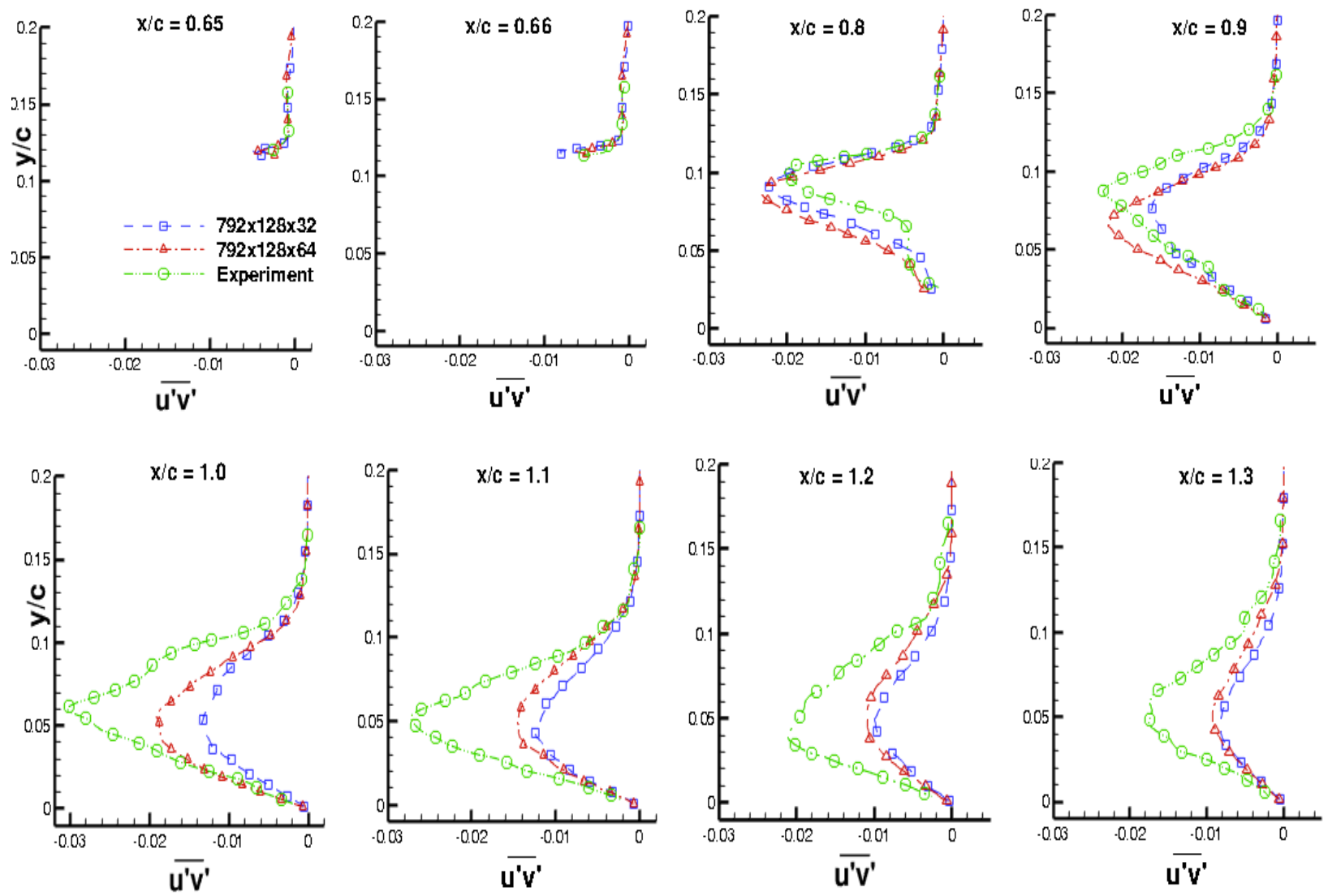

Figure 5-25: Profile of $\overline{u^{\prime} v^{\prime}}$ from WMLES on $792 \times 128 \times 32$ 
It is observed that the peak values of turbulent stresses are in better agreement with the experimental peak values than the original grid in the separated shear layer coming off the hump. At locations $\mathrm{x} / \mathrm{c}=0.9$ and 1.0, the predictions have improved in comparison to the experiment in case of $\overline{u^{\prime} u^{\prime}}$ and $\overline{u^{\prime} v^{\prime}}$ and at $\mathrm{x} / \mathrm{c}=1.1$ in the case of $\overline{v^{\prime} v^{\prime}}$. It is clear from these results that as far as the wall model is concerned, the original grid with 32 cells in the spanwise direction is adequate. However, the original grid does not quite adequately resolve the turbulent structures in the separated shear layer and an increased spanwise resolution does improve the predictions to some extent. In spite of these improvements, the original grid seems to be adequate to capture the major components of the flow physics and predicts separation and reattachment quite accurately.

\subsection{Wall resolved LES}

The previous section presented the results from a LES performed with the help of a wall model, and hence the grid used for the calculation consisted of 128 points in the $\mathrm{y}$ - direction, which yielded a value of $\Delta y^{+}$(first grid point off the wall) within $20-40$ throughout the domain. A WRLES was performed after this in order to compare the solution with the results from WMLES. For this, the number of cells in the y direction was increased to 160 , in order to satisfy the requirement $\Delta \mathrm{y}^{+}<1$ which is typical of a wall resolved LES. Further, 64 cells in the $\mathrm{z}$ direction were used in order to avoid high aspect ratio cells near the wall, giving a total resolution of $792 \mathrm{x}$ $160 \times 64$. Since the WMLES calculation performed on the grid $792 \times 128 \times 32$ were found to be satisfactory in terms of accuracy, the WRLES results presented in this section are compared to the WMLES results obtained from the same. This would give a clear idea as to how much we can reduce the computational cost for an LES. 
Figure 5-26 \& 5-27 show the comparison of calculated streamwise pressure coefficient and skin friction respectively between WMLES performed earlier and the present WRLES.

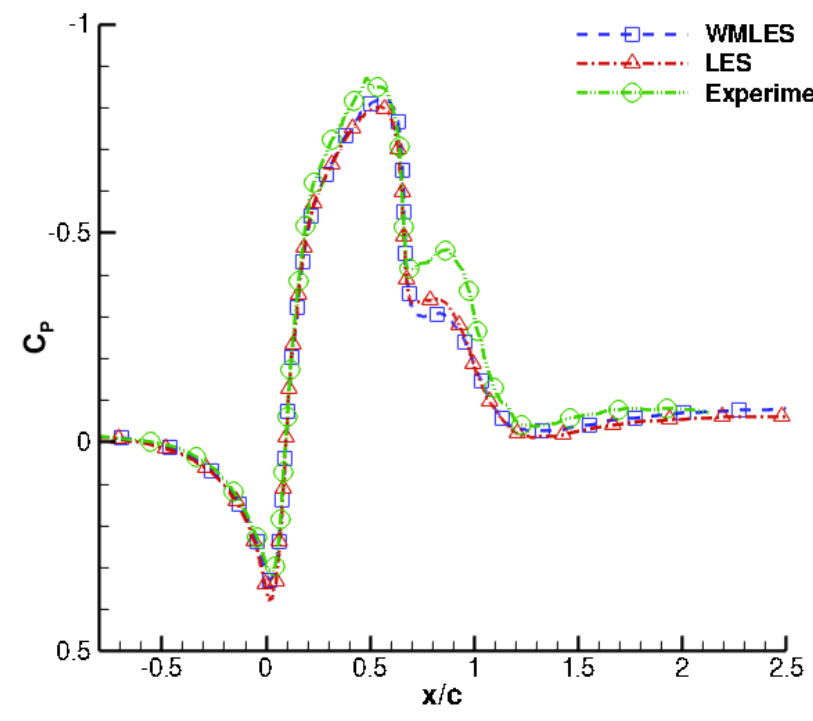

Figure 5-26: $C_{P}$ from LES

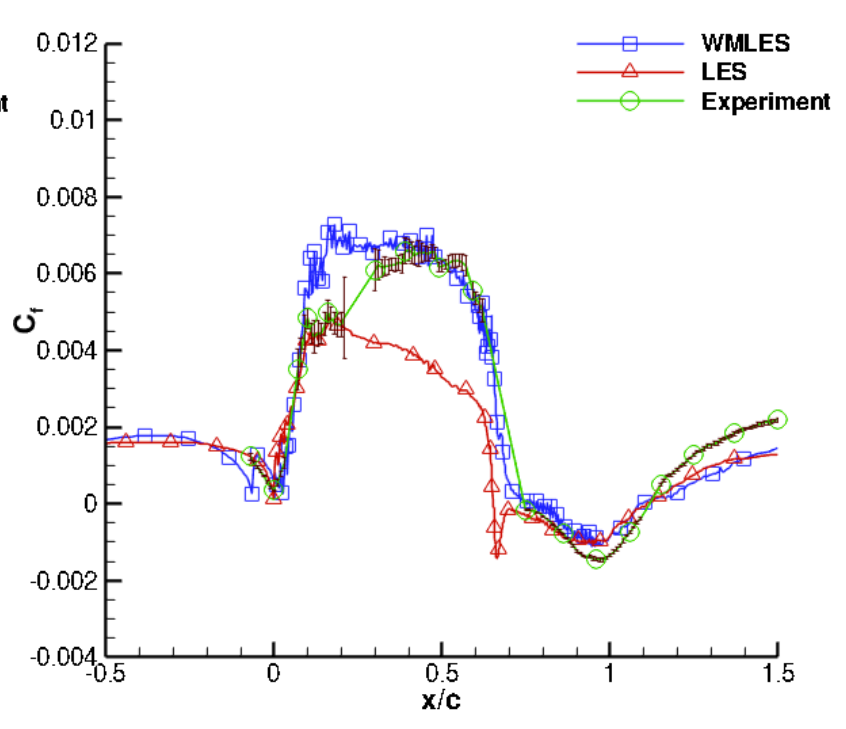

Figure 5-27: $\mathrm{C}_{\mathrm{f}}$ from LES

It can be seen that the prediction of the wall resolved LES is nearly the same as that from WMLES, with no significant difference between the two. In case of skin friction, the WRLES prediction is in agreement with the experiment up to $\mathrm{x} / \mathrm{c}=0.2$, after which the skin friction drops as the flow accelerates on the convex side of the hump, leading to under prediction of the experimentally obtained value. The WRLES profile also shows that the flow separates slightly earlier than the experiment, whereas in case of the WMLES, the separation point agrees with the experimental value. The reattachment point however, is obtained as 1.12 , which agrees with the experimental value as well as the result from WMLES. It should be noted that similar trend in $\mathrm{C}_{\mathrm{f}}$ was observed by Morgan et al. [44] in their work for regions $0.2<\mathrm{x} / \mathrm{c}<0.65$ with early separation as well. One possible reason for this could be the insufficient grid resolution in $\mathrm{x}$ - direction in this region, which is not as fine as in the region beyond $\mathrm{x} / \mathrm{c}=0.65$. A WMLES solves a separate equation within the first cell off the wall, unlike a WRLES, which is required to resolve the 
turbulent structures. Hence the lack of streamwise resolution in WMLES might not affect the accuracy, leading to a better agreement with the experimental results.

Figure 5-28 shows the WRLES prediction of mean velocity profiles at the different locations, compared against both the experimental data and the results from WMLES.
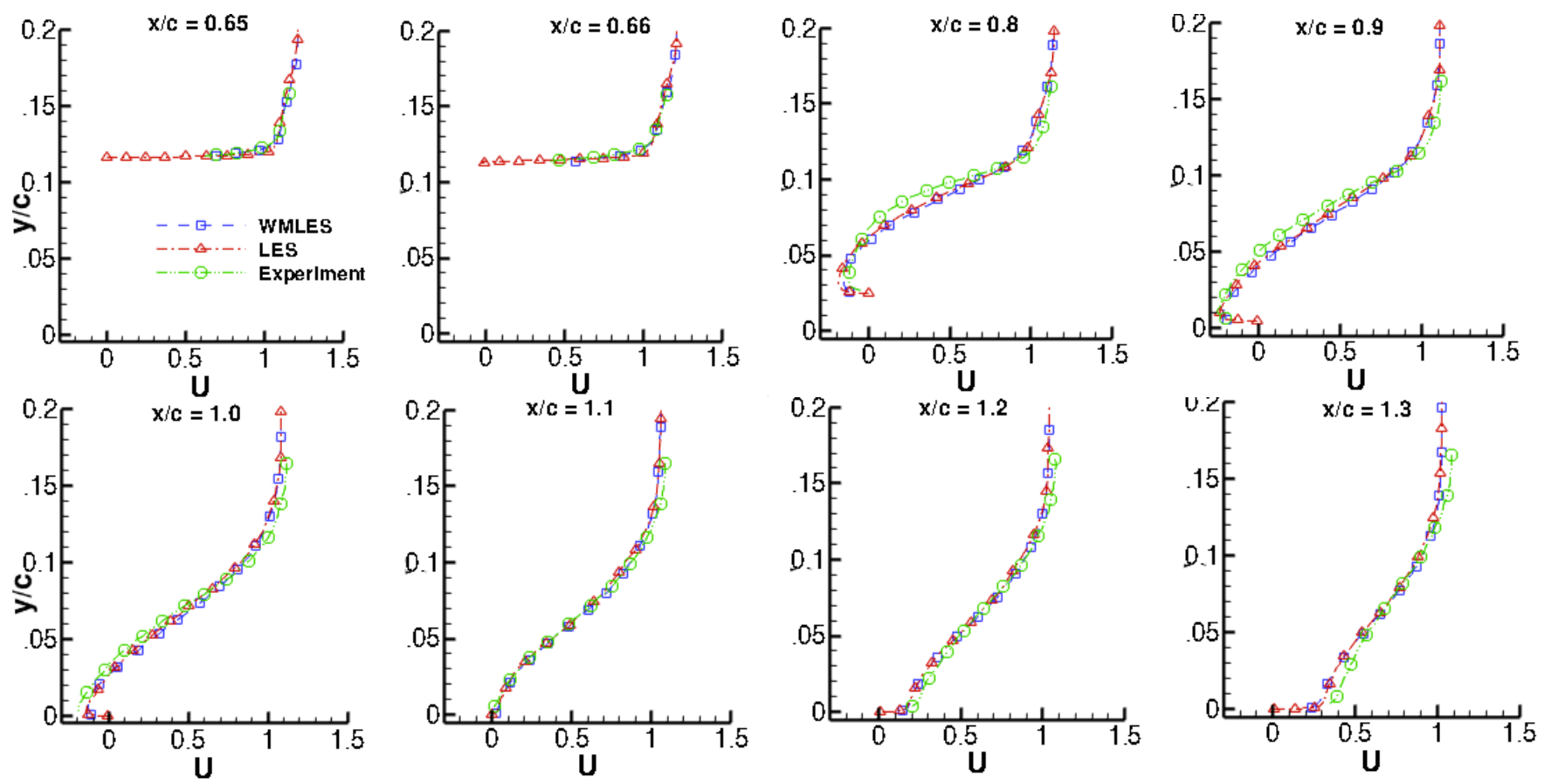

Figure 5-28: Profile of mean streamwise velocity from LES

The WRLES very closely predicts the experimental profile at all the locations as in the case of WMLES. Slight deviations from experimental data are observed at locations $0.8,0.9$ and 1.3 just as in the case of WMLES. However, these differences are too small to indicate any major difference in flow physics predicted by simulation.

Figures 5-29 - 5-31 show the comparison between the turbulent quantities obtained from the WRLES and WMLES 

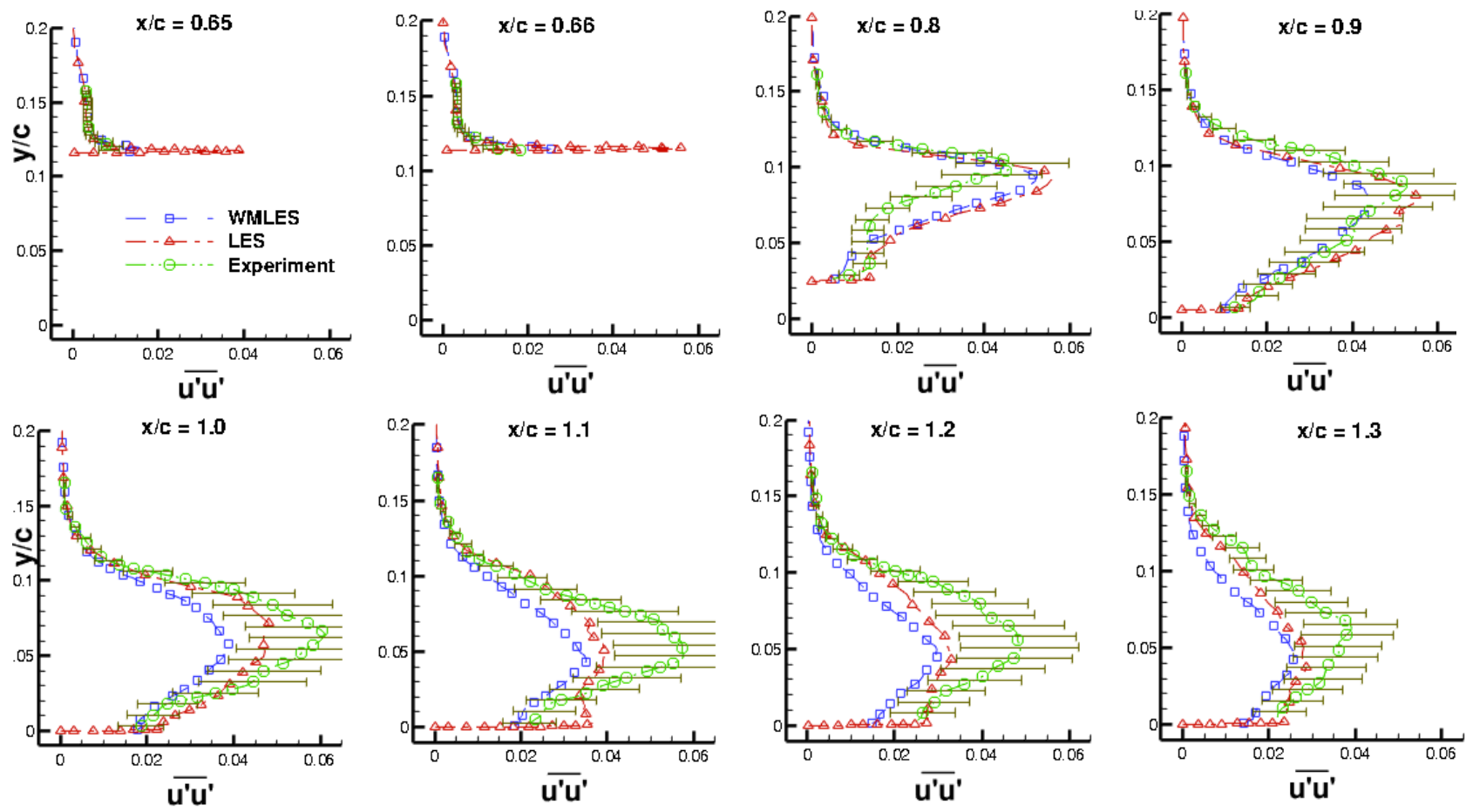

Figure 5-29: Profile of $\overline{u^{\prime} u^{\prime}}$ from LES compared with WMLES
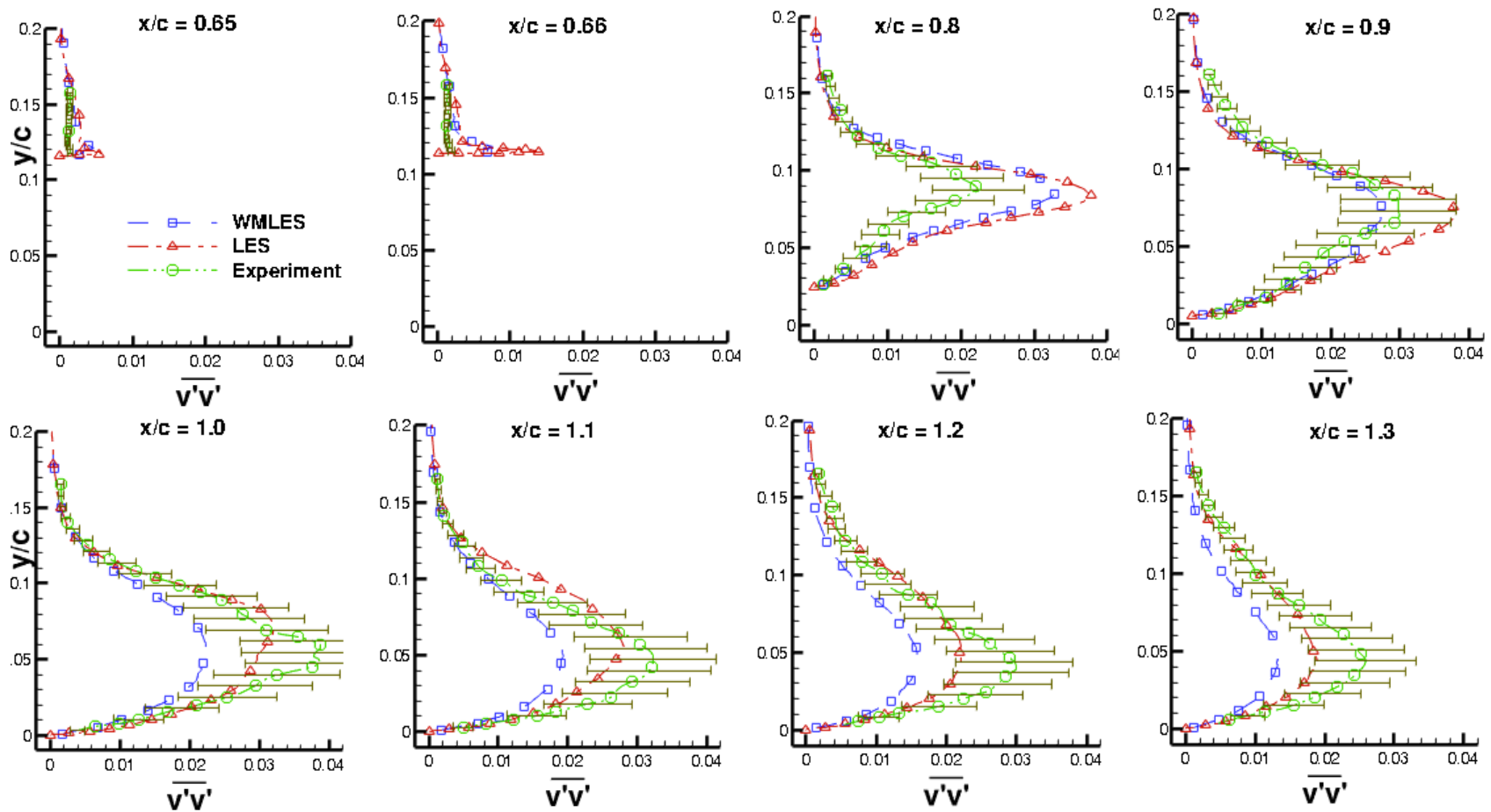

Figure 5-30: Profile of $\overline{v^{\prime} v^{\prime}}$ from LES compared with WMLES 

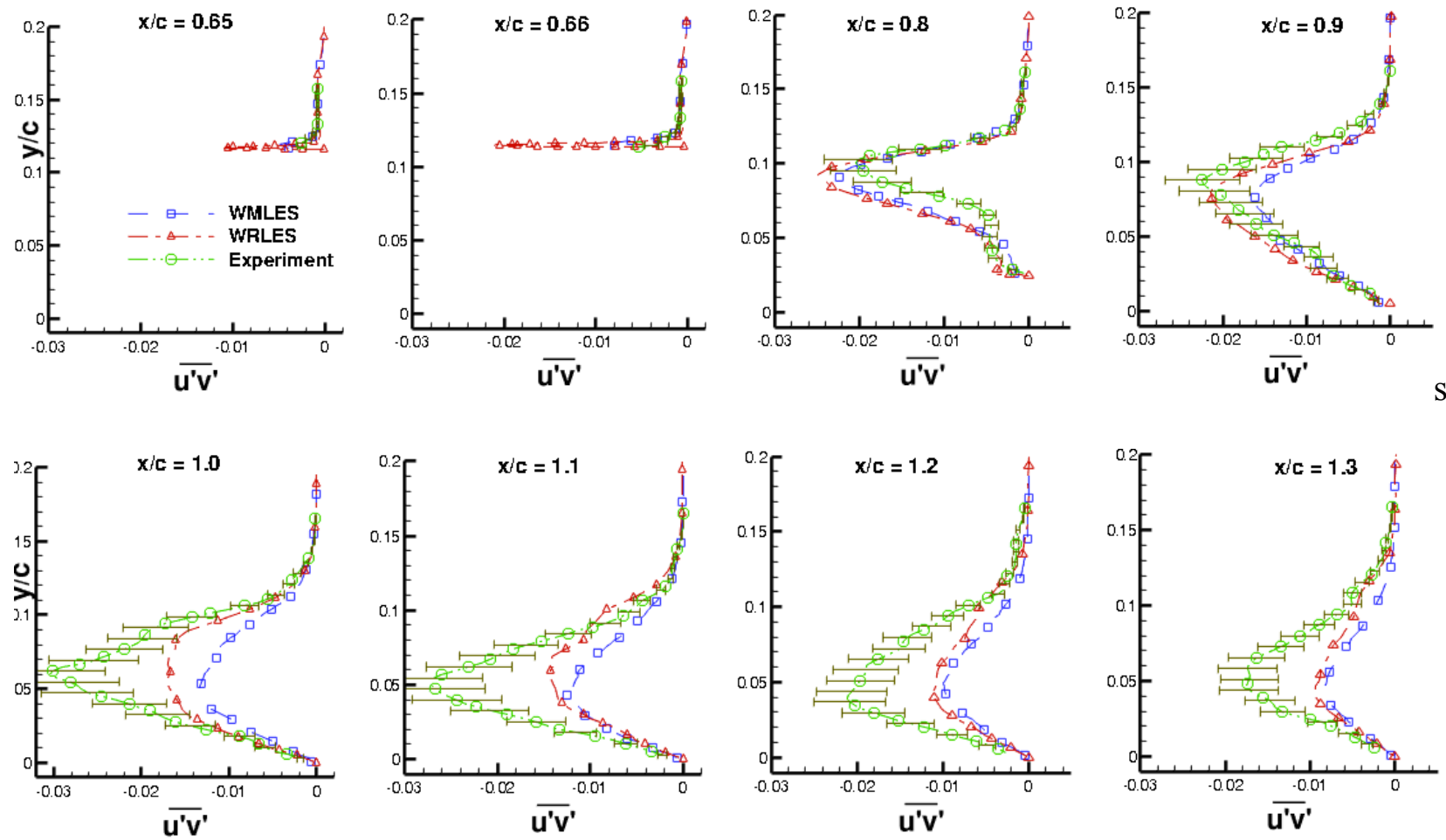

Figure 5-31: Profile of $\overline{u^{\prime} v^{\prime}}$ from LES compared with WMLES

In general, the results from WRLES agree more closely with experiments everywhere in comparison to the results from WMLES. The difference between WRLES and WMLES is more evident at some locations while at others they are approximately the same. In particular, in case of $\overline{u^{\prime} u^{\prime}}$, the WRLES gives a much better prediction of the experimental data at locations 0.9 and 1.0, which are inside the separation bubble. At $\mathrm{x} / \mathrm{c}=0.65$ and 0.66 , the over prediction in case of WRLES is higher than WMLES. Both WRLES and WMLES profiles are similar at $\mathrm{x} / \mathrm{c}=0.8$, while at $\mathrm{x} / \mathrm{c}=1.1,1.2$ and 1.3 the agreement between WRLES and experiment is marginally better than in case of WMLES.

In case of $\overline{v^{\prime} v^{\prime}}$, the WRLES fares better at predicting the experimental data than WMLES at most locations, particularly at $\mathrm{x} / \mathrm{c}=1.0,1.1,1.2$ and 1.3. Although the WRLES profiles lie within the uncertainty band at these locations, there is still some degree of under prediction with respect 
to the experimental data. However, it is not as much as in the case of WMLES. Slight over predictions are also observed at $\mathrm{x} / \mathrm{c}=0.65,0.66$, and 0.8 . At $\mathrm{x} / \mathrm{c}=0.9$, the profile from WMLES is seen to be better than that from WRLES. For $\overline{u^{\prime} v^{\prime}}$, significant improvement in WRLES prediction over WMLES prediction is seen only at $\mathrm{x} / \mathrm{c}=0.9$ and 1.0 , while at other locations, the two profiles are similar in terms of agreement with experiment. There is continued over prediction at locations $\mathrm{x} / \mathrm{c}=0.65$ and 0.66, good agreement at $\mathrm{x} / \mathrm{c}=0.8$ and 0.9 and under prediction at $\mathrm{x} / \mathrm{c}=1.0,1.1,1.2$ and 1.3.

Thus, it is observed that the WRLES is capable of predicting the turbulent parameters better than WMLES, especially in case of $\overline{v^{\prime} v^{\prime}}$, where the WRLES shows significant improvement at certain locations. However, in the case of mean velocity profiles, pressure coefficient, skin friction and reattachment length, the WMLES predictions and WRLES predictions are similar in terms of accuracy. The wall model is capable of predicting these parameters with almost the same accuracy as that of a WRLES, with a lesser grid resolution in the wall normal direction. As found in the previous section, increasing the resolution in the spanwise direction to 64 cells also increases the accuracy of WMLES similar to that observed with the wall resolved LES.

\subsection{Wall model inner layer profiles}

The use of wall model allows a higher value of $\Delta y^{+}$for the first node off the wall. As described in the previous chapter, the wall model generates a virtual grid between the wall and the first node where it calculates a velocity profile. In case of WRLES, there is no such modeling applied, and hence the first node is placed at $\Delta \mathrm{y}^{+}$less than 1 . This section compares the velocity profile generated within the inner layer by the wall model and compares it with the actual velocity profile obtained from the WRLES. For the comparison, two calculations were performed on a WRLES grid for one time step each, one with the wall model turned on and the other without the 
wall model. The boundary conditions to the wall model were obtained from the WRLES instantaneous velocity field at the $30^{\text {th }}$ node off the wall, which corresponded to a $\Delta \mathrm{y}^{+}$of between 20-40 for the entire length of the domain. The spanwise-averaged WRLES profiles are compared to the calculated spanwise-averaged WMLES profiles at the same 8 locations as before (Figures 5$32-5-34)$
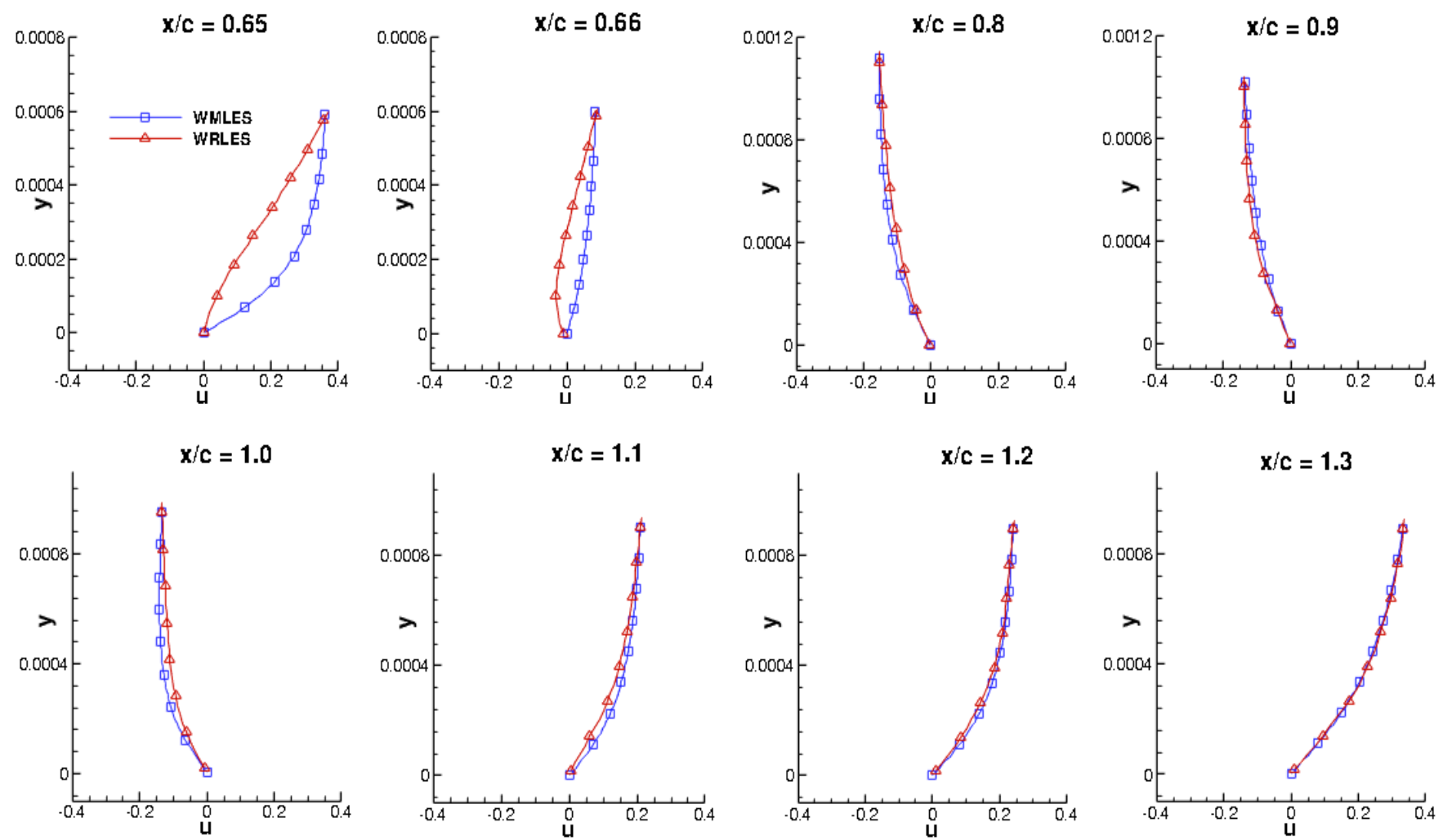

Figure 5-32: Comparison of profile of u from LES and WMLES 

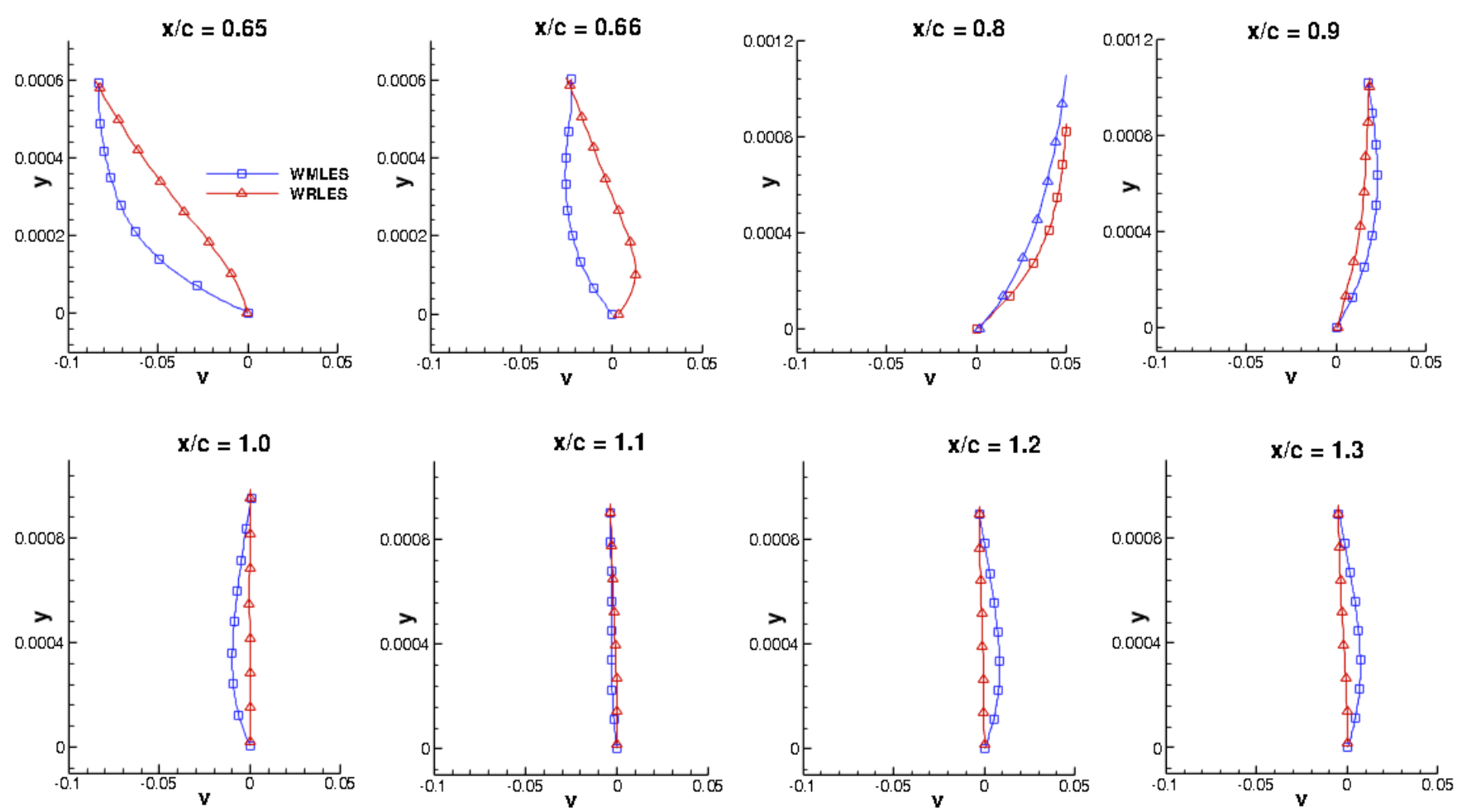

Figure 5-33: Comparison of profile of $v$ from LES and WMLES
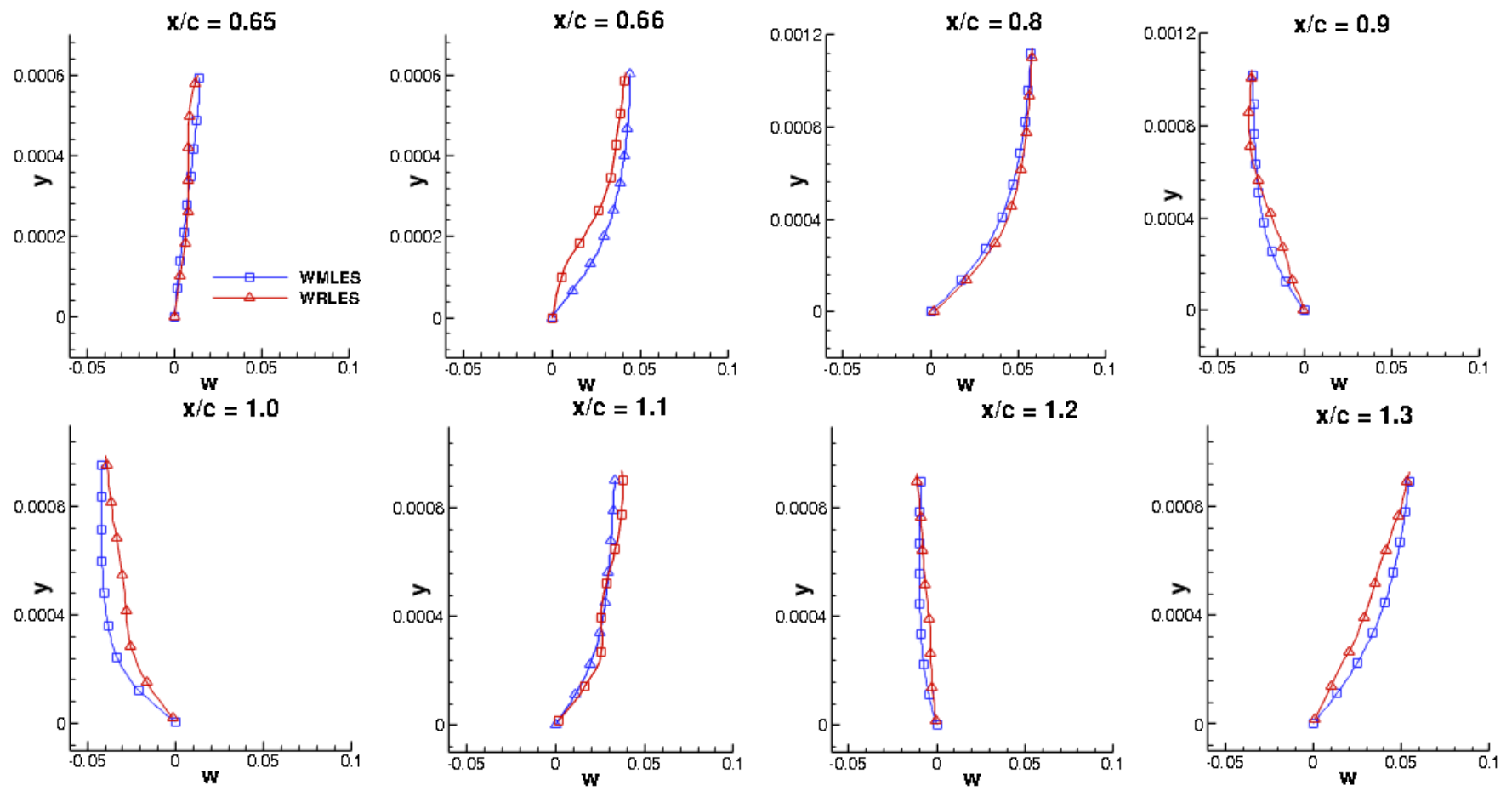

Figure 5-34: Comparison of profile of $w$ from LES and WMLES 
The plots above show the comparison of profiles of streamwise, normal and spanwise component of the velocity obtained from using the wall model, compared to those from a wall resolved calculation. The velocities shown are instantaneous, averaged over the entire span of the domain. It can be seen that the profile predicted by the wall model matches closely with the profile from LES at most points in case of all the velocity components. The only exceptions are at locations 0.65 and 0.66 in case of $u$ and v velocity. Observing the profile of $u$ and $v$ at location 0.66 indicate that the early prediction of separation by LES relative to the wall model. This could indicate that the magnitude of adverse pressure gradient in the tangential direction at this point is not large enough to induce separation. Beyond this location, the profiles from WRLES and WMLES agree closely, indicating the ability of the wall model to predict the inner layer profile quite accurately. 


\section{Conclusions \& Recommendations}

Wall modeled LES has been found to be an effective and accurate method of simulating turbulent flows at high Reynolds numbers without the typical computational cost associated with a regular LES. The investigations were conducted by simulating the turbulent flow of Reynolds number approximately 1 million over a wall-mounted hump. The wall modeling allowed the use

of large eddy simulation on a grid having the first off-wall grid point at a $\mathrm{y}^{+}$of approximately 20 40, thereby relaxing the stringent grid requirements of a WRLES. The results obtained from a WMLES were compared to the experimental data as well as the results from a WRLES. It was observed that the WMLES predicted various parameters such as reattachment location, mean velocity profiles, streamwise variation of skin friction and coefficient of pressure with good accuracy in comparison to both the experimental data as well as WRLES. There was some degree of under prediction of the turbulent quantities close to the reattachment location while the agreement was better in the separated region of the flow. The same trend is exhibited by results from the WRLES calculation. On the other hand, the results from a calculation performed without enabling the wall model on the same grid used for WMLES showed large discrepancies with the experimental data. In this case, the calculation failed to predict the flow separation at the concave portion of the hump. It was observed subsequently that the wall modeling also allowed a reduction in the number of cells in the spanwise by half without suffering loss in accuracy which further reduced the grid size. A calculation attempted at performing a WRLES on the grid with reduced number of cells in spanwise turned unstable immediately, thereby further emphasizing the effectiveness of wall modeling. The relaxed grid resolution for the wall modeled LES not only reduced the number of grid points in the spatial domain but also permitted a higher time 
step for the calculation. In total, the total CPU time required for a WMLES calculation was observed to be an order of magnitude lower than a wall-resolved LES.

As for recommendations, the validation of the wall model can be extended to a flow that is three dimensional in nature. The current problem involved a nominally two-dimensional flow with pressure gradient in one dimension. A more complicated case would be the FAITH experiment [57], where the flow is characterized by strong three-dimensional favorable and adverse pressure gradients. This will provide a good test case to develop and validate the wall model further for complex three-dimensional separation. Once the aforementioned validation is done, the wall model can be employed in simulating non-stationary flow phenomenon such as the dynamic stall of airfoils and thus can alleviate the extreme computational resource requirement of an accurate wall resolved LES that makes the required phase averaging almost impossible from a practical point of view. 


\section{References}

1. Chapman, D.K., Computational aerodynamics development and outlook. AIAA journal, 1979. 17(12): p. 1293-1313.

2. Deardorff, J.W., A numerical study of three-dimensional turbulent channel flow at large Reynolds numbers. J. Fluid Mech, 1970. 41(2): p. 453-480.

3. Schumann, U., Subgrid scale model for finite difference simulations of turbulent flows in plane channels and annuli. Journal of computational physics, 1975. 18(4): p. 376404.

4. Spalart, P., et al., Comments on the feasibility of LES for wings, and on a hybrid \{RANS/LES\} approach. 1997.

5. SPALART, P. and S. ALLMARAS, A one-equation turbulence model for aerodynamic flows. 1992.

6. Nikitin, N., et al., An approach to wall modeling in large-eddy simulations. Physics of Fluids, 2000.12(7): p. 1629-1632.

7. Strelets, M., Detached eddy simulation of massively separated flows. 2001: American Institute of Aeronautics \& Astronautics.

8. S Šarić, S., et al., Comparative assessment of hybrid LES/RANS models in turbulent flows separating from smooth surfaces, in Advances in Hybrid RANS-LES Modelling. 2008, Springer. p. 142-151.

9. Viswanathan, A.K. and D.K. Tafti, Capturing effects of rotation in sudden expansion channels using detached eddy simulation. AIAA journal, 2007. 45(8): p. 2100-2102.

10. Viswanathan, A.K. and D.K. Tafti, A comparative study of DES and URANS for flow prediction in a two-pass internal cooling duct. Journal of fluids engineering, 2006. 
128(6): p. 1336-1345.

11. Viswanathan, A.K. and D.K. Tafti, Detached eddy simulation of turbulent flow and heat transfer in a two-pass internal cooling duct. International Journal of Heat and Fluid Flow, 2006. 27(1): p. 1-20.

12. Cabot, W. and P. Moin, Approximate wall boundary conditions in the large-eddy simulation of high Reynolds number flow. Flow, Turbulence and Combustion, 2000. 63(1-4): p. 269-291.

13. Wang, M. and P. Moin, Dynamic wall modeling for large-eddy simulation of complex turbulent flows. Physics of Fluids (1994-present), 2002. 14(7): p. 2043-2051.

14. Tessicini, F., N. Li, and M. Leschziner, Large-eddy simulation of three-dimensional flow around a hill-shaped obstruction with a zonal near-wall approximation. International Journal of Heat and Fluid Flow, 2007. 28(5): p. 894-908.

15. Patil, S. and D. Tafti, Wall modeled large eddy simulations of complex high Reynolds number flows with synthetic inlet turbulence. International Journal of Heat and Fluid Flow, 2012. 33(1): p. 9-21.

16. Patil, S. and D. Tafti, Large-Eddy Simulation of Flow and Convective Heat Transfer in a Gas Turbine Can Combustor With Synthetic Inlet Turbulence. Journal of Engineering for Gas Turbines and Power, 2012. 134(7): p. 071503.

17. Patil, S. and D. Tafti, Large-eddy simulation with zonal near wall treatment of flow and heat transfer in a ribbed duct for the internal cooling of turbine blades. Journal of Turbomachinery, 2013. 135(3): p. 031006.

18. Greenblatt, D., et al., A separation control CFD validation test case, Part 1: baseline and steady suction. AIAA paper, 2004. 2220: p. 2004.

19. Greenblatt, D., et al., A Separation Control CFD Validation Test Case Part 2: Zero Efflux 
Oscillatory Blowing. AIAA paper, 2005. 485: p. 43.

20. Naughton, J.W., S. Viken, and D. Greenblatt, Skin friction measurements on the NASA hump model. AIAA journal, 2006. 44(6): p. 1255-1265.

21. Seifert, A. and L.G. Pack, Active flow separation control on wall-mounted hump at high Reynolds numbers. AIAA journal, 2002. 40(7): p. 1363-1372.

22. Launder, B. and B. Sharma, Application of the energy-dissipation model of turbulence to the calculation of flow near a spinning disc. Letters in heat and mass transfer, 1974. 1(2): p. 131-137.

23. Abe, K., T. Kondoh, and Y. Nagano, A new turbulence model for predicting fluid flow and heat transfer in separating and reattaching flows-I. Flow field calculations. International Journal of Heat and Mass Transfer, 1994. 37(1): p. 139-151.

24. Abe, K., Y.-J. Jang, and M.A. Leschziner, An investigation of wall-anisotropy expressions and length-scale equations for non-linear eddy-viscosity models. International Journal of Heat and Fluid Flow, 2003. 24(2): p. 181-198.

25. Balakumar, P. Computations of flow over a hump model using higher order method with turbulence modeling. in 43 rd AIAA Aerospace Sciences Meeting and Exhibit. 2005.

26. Cui, J. and R. Agarwal, CFD Validation of Turbulent Separation Control on a 2D Hump (NASA Langley Workshop Validation: Case 3). AIAA Paper, 2005. 5013.

27. Hiller, S.J. and P.A. Seitz, The Interaction between a fluidic actuator and main flow using SAS turbulence modeling. AIAA Paper, 2006. 3678: p. 2006.

28. Madugundi, D., H. Nagib, and J. Kiedaisch, Evaluation of Turbulence Models Through Prediction of Separated Flows with and without Flow Control and Circulation Effects. AIAA, 2008. 567: p. 2008. 
29. Yoshio, M. and K.-i. Abe, Performance of Reynolds-Averaged Turbulence Models for Unsteady Separated Flows with Periodic Blowing and Suction, in Advances in Turbulence XI. 2007, Springer. p. 723-723.

30. Yeshala, N., B. Min, and L. Sankar. Drag reduction studies using active flow control. in 4th AIAA Flow Control Conference, Washington, DC. 2008.

31. He, C., T.C. Corke, and M.P. Patel, Numerical and experimental analysis of plasma flow control over a hump model. AIAA paper, 2007. 935: p. 8-11.

32. Krishnan, V., K.D. Squires, and J.R. Forsythe, Prediction of separated flow characteristics over a hump using RANS and DES. AIAA Paper, 2004. 2224.

33. Rumsey, C.L., Reynolds-averaged Navier-Stokes analysis of zero efflux flow control over a hump model. Journal of aircraft, 2007. 44(2): p. 444-452.

34. Shakib, F., D. Corson, and R. Jaiman, Industrial Application of RANS Modelling: Capabilities and Needs. 2013.

35. Postl, D. and H.F. Fasel, Direct numerical simulation of turbulent flow separation from a wall-mounted hump. AIAA journal, 2006. 44(2): p. 263-272.

36. Šarić, S., et al., Computational analysis of locally forced flow over a wall-mounted hump at high $-<i>\operatorname{Re}</ i>$ number. International journal of heat and fluid flow, 2006. 27(4): p. 707-720.

37. Travin, A., et al., Physical and numerical upgrades in the detached-eddy simulation of complex turbulent flows, in Advances in LES of complex flows. 2004, Springer. p. 239254.

38. Spalart, P.R., et al., A new version of detached-eddy simulation, resistant to ambiguous grid densities. Theoretical and computational fluid dynamics, 2006. 20(3): p. 181195. 
39. Jakirlić, S., et al. Merging near-wall RANS models with LES for separating and reattaching flows. in ASME 2006 2nd Joint US-European Fluids Engineering Summer Meeting Collocated With the 14th International Conference on Nuclear Engineering. 2006. American Society of Mechanical Engineers.

40. Smagorinsky, J., General circulation experiments with the primitive equations: I. The basic experiment*. Monthly weather review, 1963. 91(3): p. 99-164.

41. Jakirlic, S. and K. Hanjalic, A new approach to modelling near-wall turbulence energy and stress dissipation. Journal of fluid mechanics, 2002. 459: p. 139-166.

42. Lyons, D.C., et al., Assessment of DES models for separated flow from a hump in a turbulent boundary layer. Journal of Fluids Engineering, 2009. 131(11): p. 111203.

43. Slimon, S. Computation of internal separated flows using a zonal detached eddy simulation approach. in ASME 2003 International Mechanical Engineering Congress and Exposition. 2003. American Society of Mechanical Engineers.

44. Morgan, P.E., D.P. Rizzetta, and M.R. Visbal, Large-eddy simulation of flow over a wallmounted hump. AIAA Paper, 2005. 484.

45. Franck, J.A. and T. Colonius, Large-Eddy Simulation of Separation Control for Compressible Flow over a Wall-Mounted Hump. AIAA Paper, 2008. 555.

46. You, D., M. Wang, and P. Moin, Large-eddy simulation of flow over a wall-mounted hump with separation control. AIAA journal, 2006. 44(11): p. 2571-2577.

47. Germano, M., et al., A dynamic subgrid - scale eddy viscosity model. Physics of Fluids A: Fluid Dynamics (1989-1993), 1991. 3(7): p. 1760-1765.

48. Lilly, D., A proposed modification of the Germano subgrid - scale closure method. Physics of Fluids A: Fluid Dynamics (1989-1993), 1992. 4(3): p. 633-635. 
49. Lund, T.S., X. Wu, and K.D. Squires, Generation of turbulent inflow data for spatiallydeveloping boundary layer simulations. Journal of Computational Physics, 1998. $140(2):$ p. $233-258$.

50. Avdis, A., S. Lardeau, and M. Leschziner, Large eddy simulation of separated flow over a two-dimensional hump with and without control by means of a synthetic slot-jet. Flow, turbulence and combustion, 2009. 83(3): p. 343-370.

51. Biswas, D., Studies on Separation Control CFD Validation Test Case Based on a Higher Order LES Model. AIAA Paper, 2006. 2881.

52. Noelting, S., et al., Lattice Boltzmann Simulations of the Flow over a Hump with Flow Control. 2008.

53. Tafti, D., Genidlest: A Scalable Parallel Computational Tool for Simulating Complex Turbulent Flows. ASME-PUBLICATIONS-FED, 2001. 256: p. 347-356.

54. Johnson, D. and L. King, A new turbulence closure model for boundary layer flows with strong adverse pressure gradients and separation. AIAA paper, 1984. 175.

55. Perry, A., T. Lim, and M. Chong, The instantaneous velocity fields of coherent structures in coflowing jets and wakes. Journal of fluid Mechanics, 1980. 101(02): p. $243-256$.

56. Klebanoff, P., Characteristics of turbulence in a boundary layer with zero pressure gradient. 1955: National Advisory Committee for Aeronautics.

57. Bell, J.H., et al., Surface and Flow Field Measurements on the FAITH Hill Model. NASA Ames, 2012, 2012. 


\section{Appendix - Effects of accounting for end plates and grid refinement in wall normal direction}

\section{A.1 Effect of accounting for end plates}

The actual experimental setup consisted of the wall-mounted hump enclosed between two end plates. However, the computational domain used in this work extended for only a part of the total span of the original model, and hence the end plates are not included in the geometry. A consensus was reached between the participants at the NASA workshop that there was something in the experimental set up that caused a wind tunnel "blockage", which must be accounted for in the simulation. The blockage effect can be the reason for the slight acceleration of the fluid in the region rear separation, creating a suction peak in the streamwise variation of pressure coefficient. As mentioned earlier, some previous computational studies on the same problem also observed that accounting for the blockage effect in the computational domain led to better prediction of the suction peak near the reattachment point. Hence, a case with modifications made to the top wall of the computational domain was simulated, to check if there was any difference in the results obtained. This section presents the results from a wall resolved LES performed on this domain, with all other parameters related to the calculation kept same as the previous LES.

\section{A.1.1 Modification to the computational domain}

Figure A-1 below shows the computational domain with the top wall contoured to account for the blockage created by the end plates. The top wall of the new domain is scaled by a factor equal to the approximate area of the end plates divided by the local tunnel cross-section area. The case data provided by NASA included the profile of the contour for the top wall as well. 


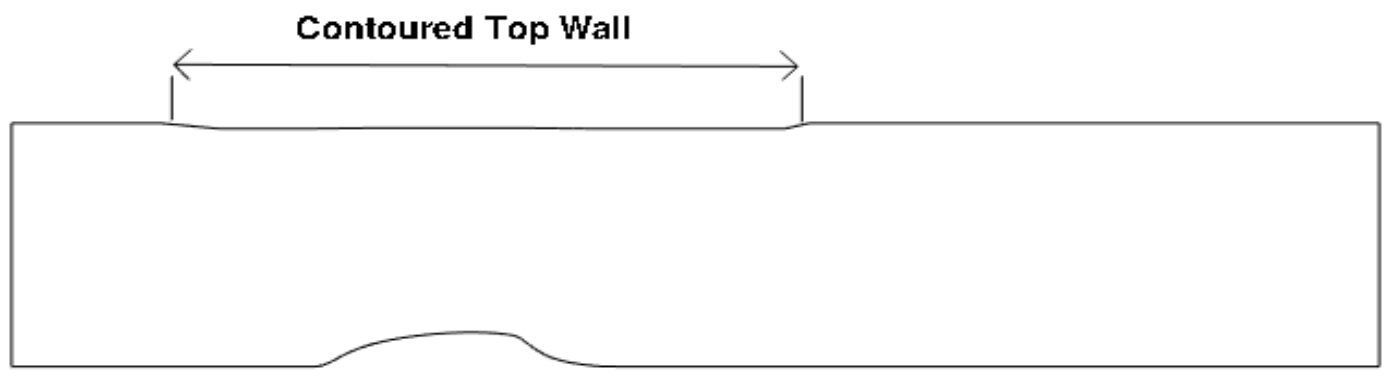

Figure A-1: Computational domain with modified top wall.

Figure A-2 shows the plot of streamwise variation of coefficient of pressure obtained from the present case, compared against the LES results presented earlier as well as the experimental data.

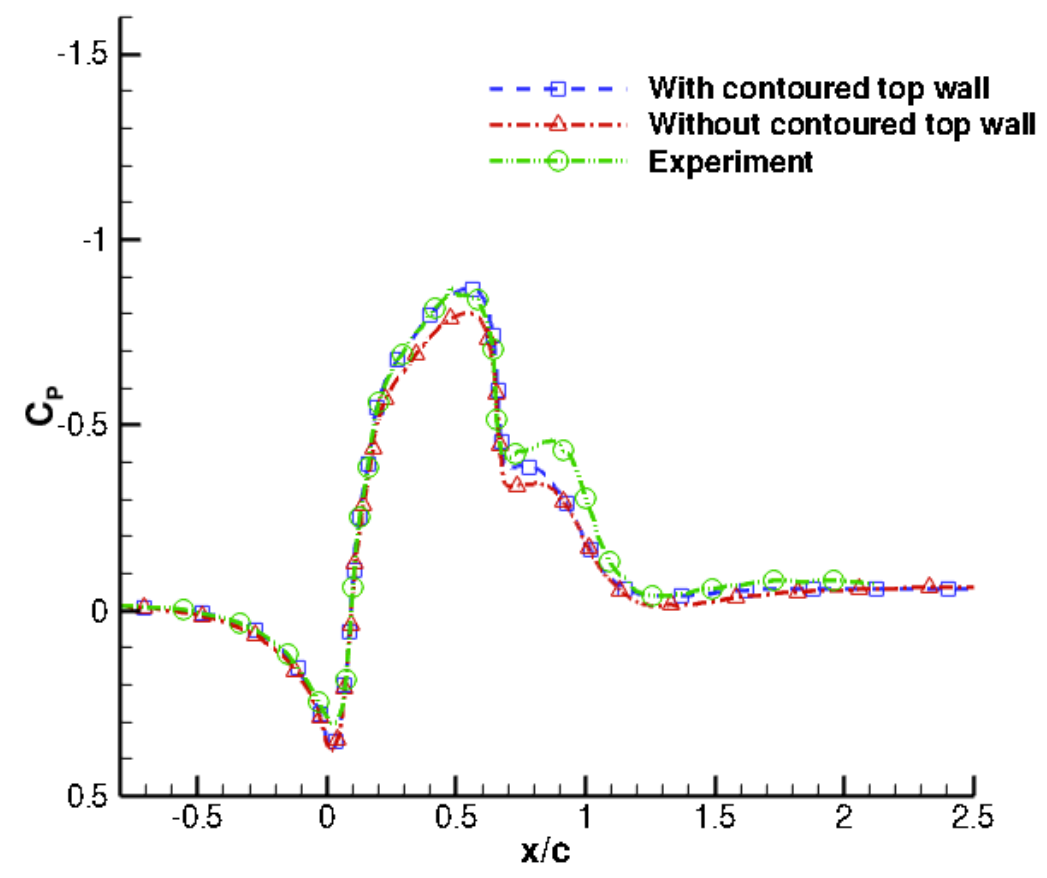

Figure A-2: Profile of $C_{P}$

As it can be seen from the plot, the alteration to the geometry of the top wall has an impact on the pressure distribution, with the peak value of suction agreeing more with the experiment than without alteration. However, the increase in suction near the separation point is only marginal with the modified geometry, with discrepancies still existing with the experimental data. The 
magnitude of adverse pressure gradient is still the same as before, in the vicinity of the separated region.

Figures A-3 - A-5 show the profile of Reynolds stresses obtained from the current simulation compared against the LES results presented earlier and the experimental data.
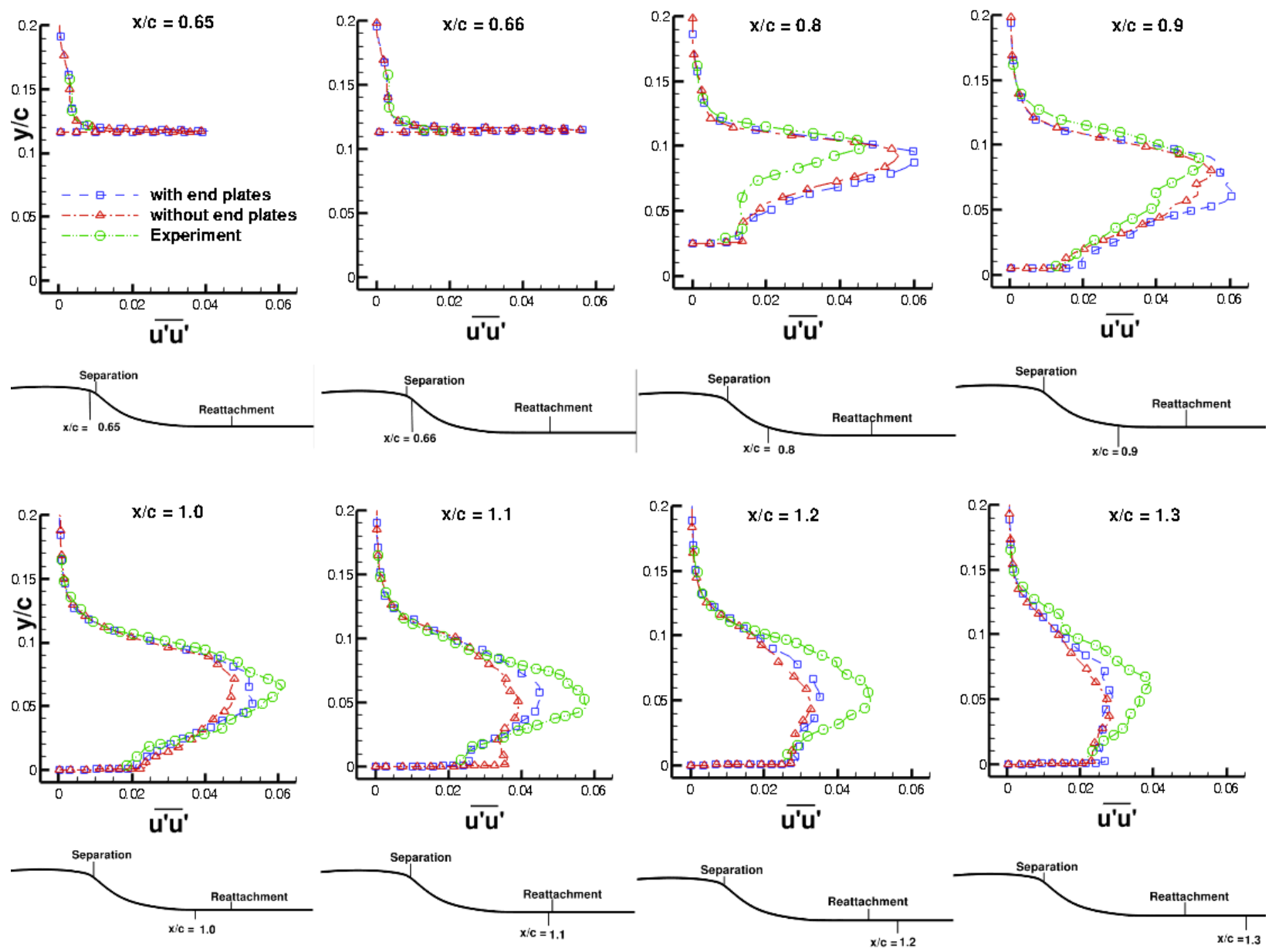

Figure A-3: Profile of $\overline{\boldsymbol{u}^{\prime} \boldsymbol{u}^{\prime}}$ 

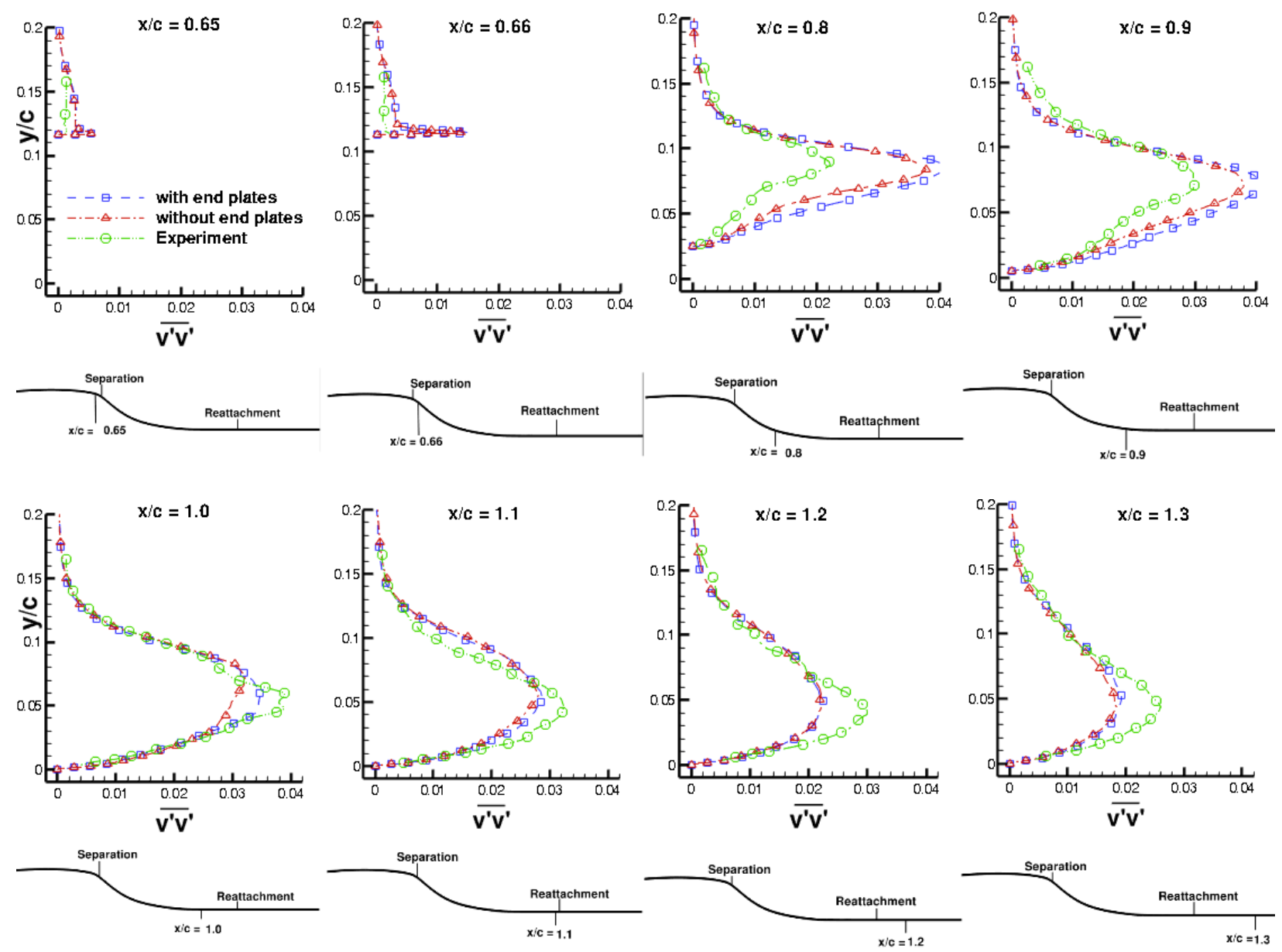

Figure A-4: Profile of $\overline{v^{\prime} u^{\prime}}$
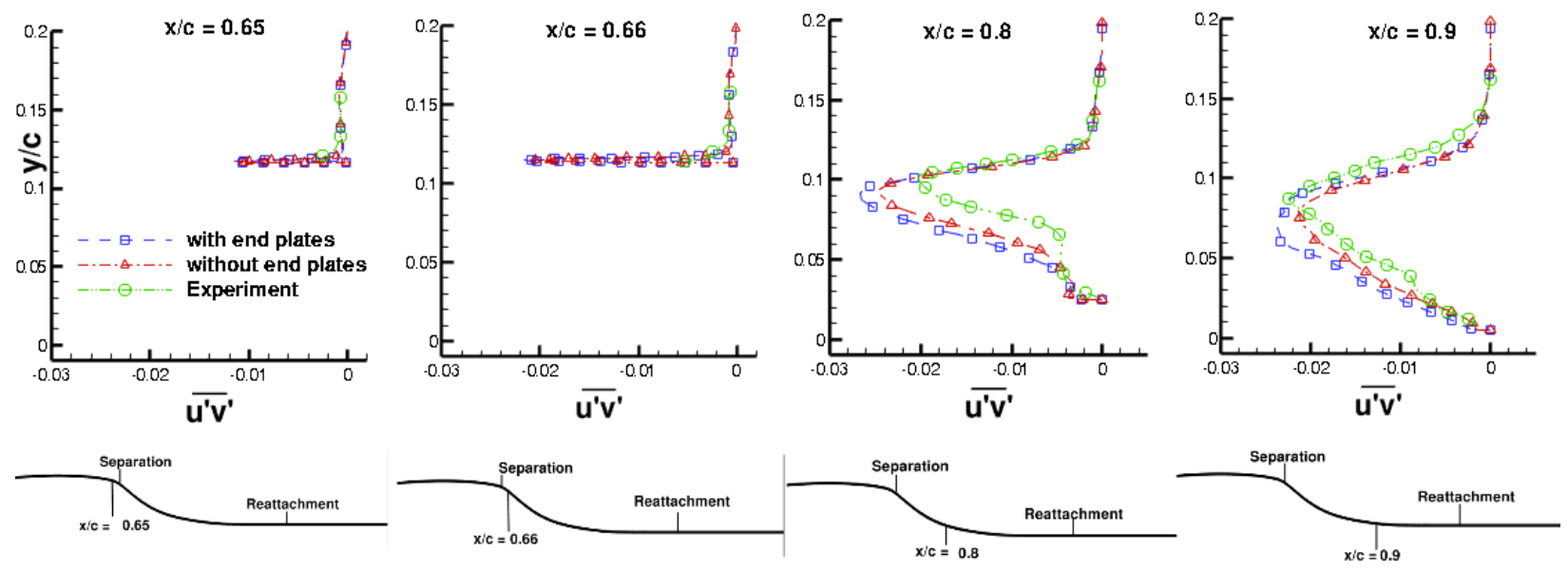

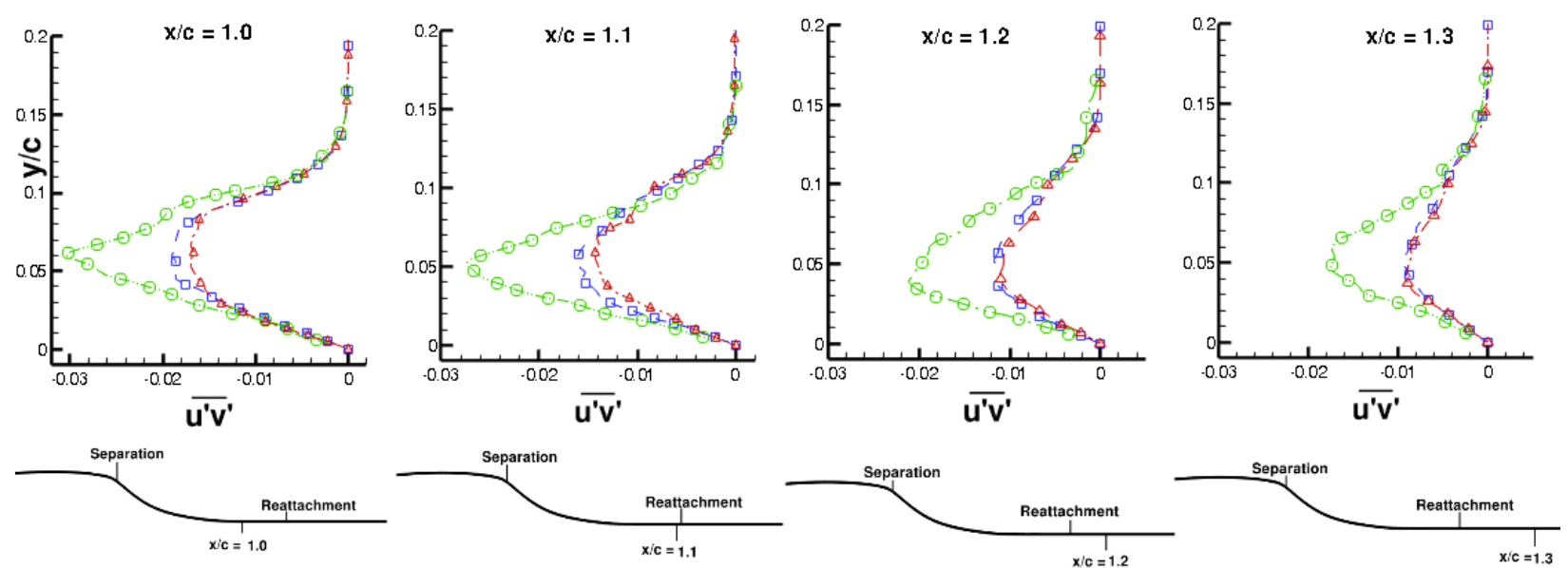

Figure A-5: Profile of $\overline{u^{\prime} v^{\prime}}$

It is seen than there is a slight increase in the peak values of the turbulent normal and shear stresses in the region beyond separation, which can be seen up to the reattachment location, indicating a marginal increase in the level of turbulence. However, the discrepancies with the experiment do not decrease significantly. A possible reason for the simulation being mostly insensitive to the change in geometry could be that the insufficiency of the magnitude by which the top wall that is scaled down to account for the blockage, and hence match the level of suction near the separation point. The pressure gradient in the region immediately downstream remains unchanged which could be affecting the turbulence.

\section{A.2 Effect of grid refinement in wall normal direction}

The grid used for all the WMLES calculations had the first off-wall node placed at a distance to yield $\mathrm{y}^{+}$of approximately 20-40. Uniform grid spacing followed this between 20-40 wall units for the subsequent 25 grid cells normal to the wall. Since the wall model is only applied between the first grid point and the wall and a regular LES is performed outside of this region, it was deemed necessary to investigate whether a finer grid resolution outside of the first grid cell adjacent to the wall would have any impact on the prediction accuracy. 
This section presents results from an investigation into this aspect. For this, the WMLES grid was modified such that the resolution beyond the first off-wall point was exactly the same as the resolution of the grid used for LES calculations (128 cells in the y-direction). This amounted to the first off-wall grid point at a distance of 20-40 wall units for the application of the wall model, followed by a fine grid of spacing equal to approximately 1 wall unit. To keep the computational cost within reasonable limits, the grid with 32 cells in the z-direction was used for the calculation. Hence the grid size is still $792 \times 128 \times 32$. All the other parameters of the simulation were kept the same as before.

Figure A-6 \& A-7 show streamwise variation of the coefficient of pressure and skin friction obtained respectively from the new simulation, compared against the WMLES results presented earlier, as well as with the experimental data.

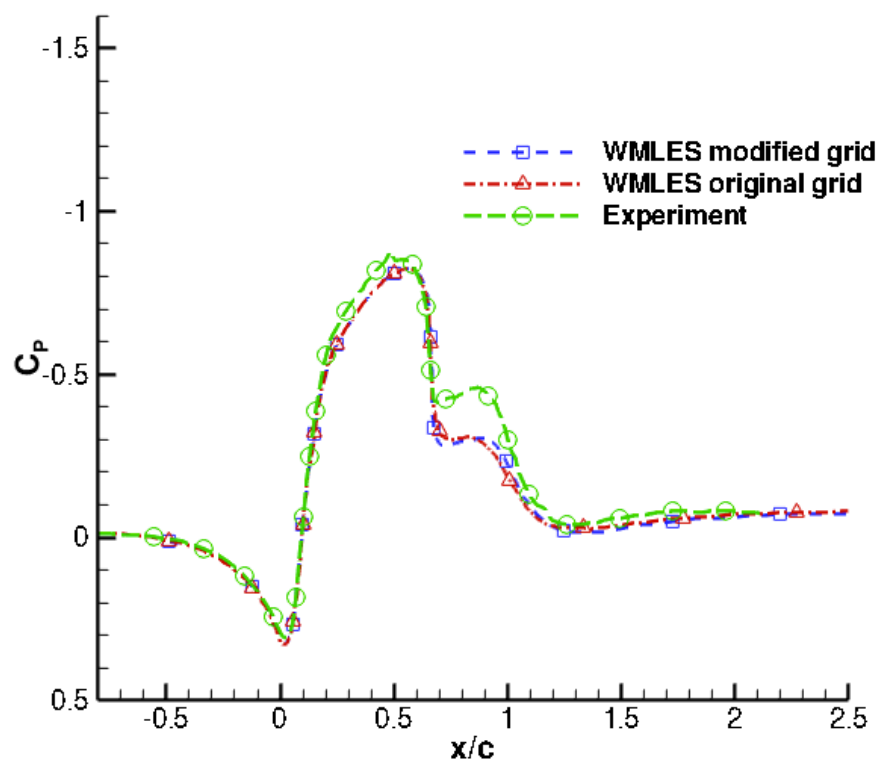

Figure A-6: Profile of $C_{P}$

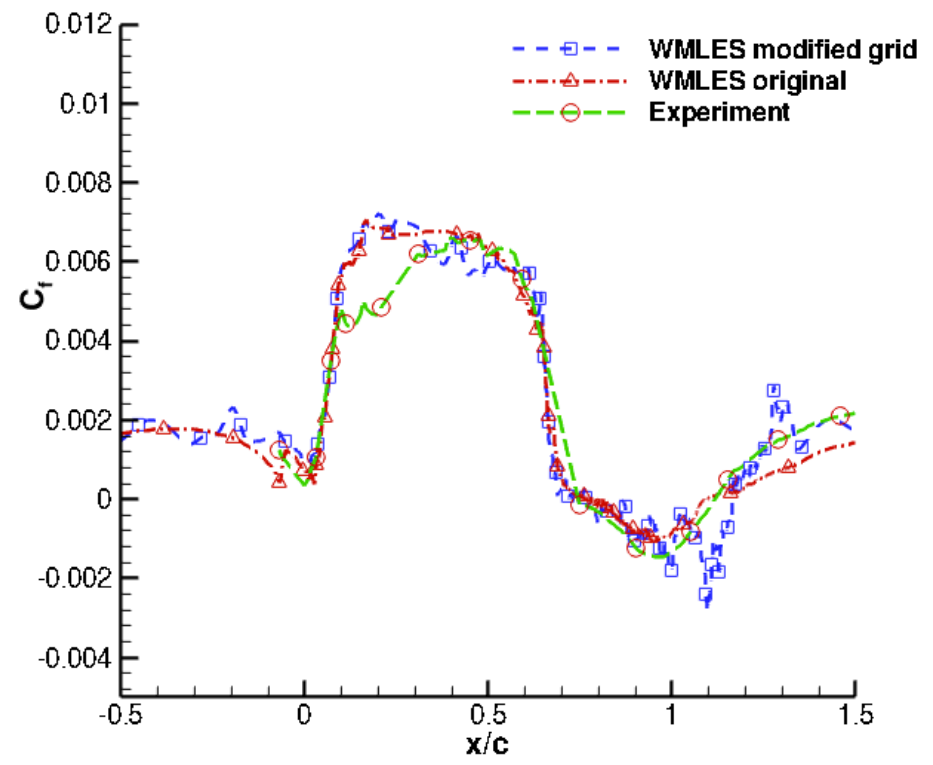

Figure A-7: Profile of $\mathbf{C}_{\mathrm{f}}$

It can be seen from the plot that change in grid resolution has negligible impact on the variation of pressure coefficient. In case of skin friction coefficient, the plots from the two cases agree for 
the most part, with slightly higher negative values on the modified grid, just upstream of reattachment. The reattachment location is obtained as 1.16 for the current simulation.

Figures A8 - A10 show the profiles of Reynolds stresses and figure A-11 shows the profile of mean streamwise velocities obtained from the current simulation compared against the WMLES results presented earlier and the experimental data.
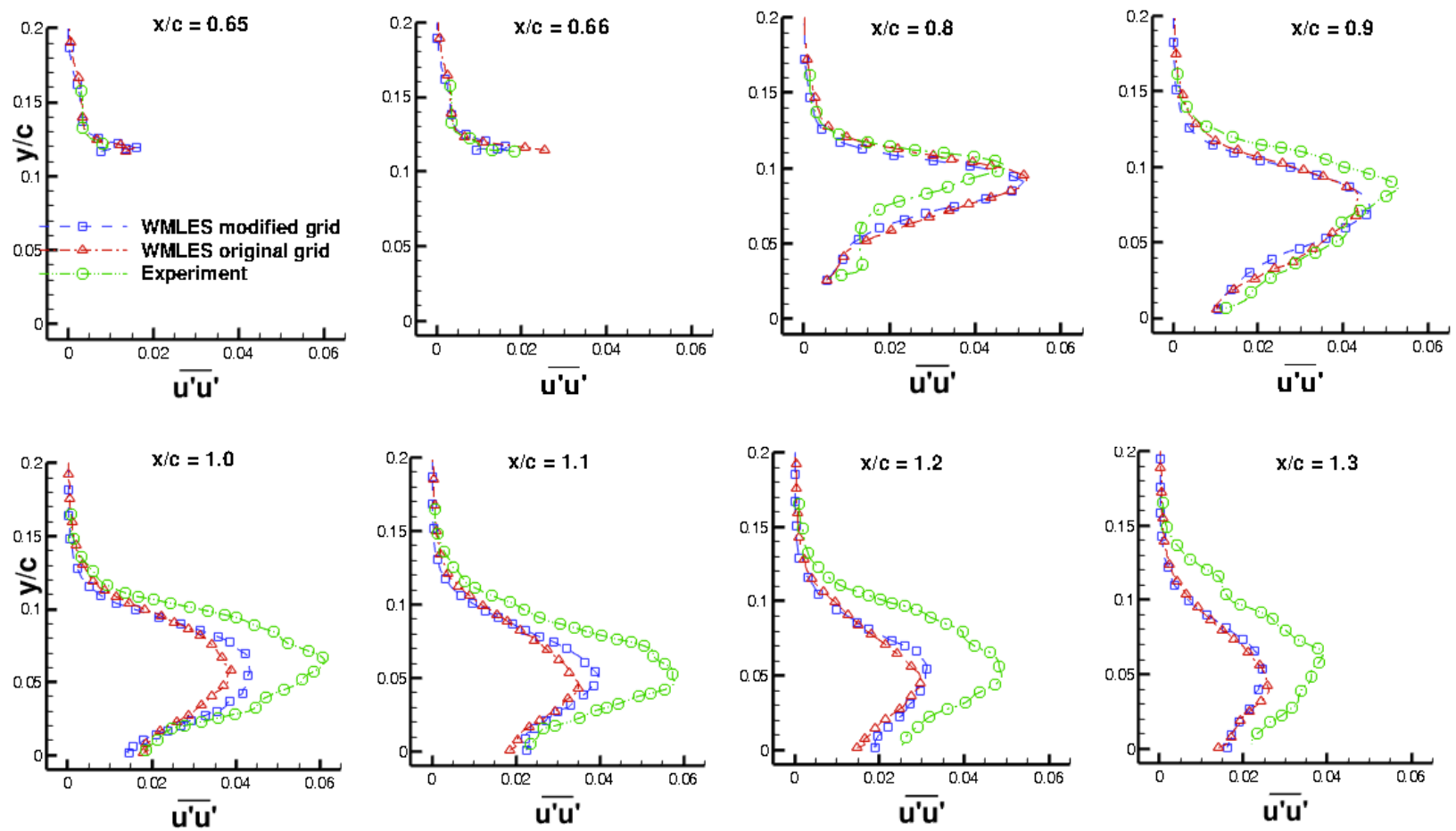

Figure A-8: Profile of $\overline{\boldsymbol{u}^{\prime} \boldsymbol{u}^{\prime}}$ 

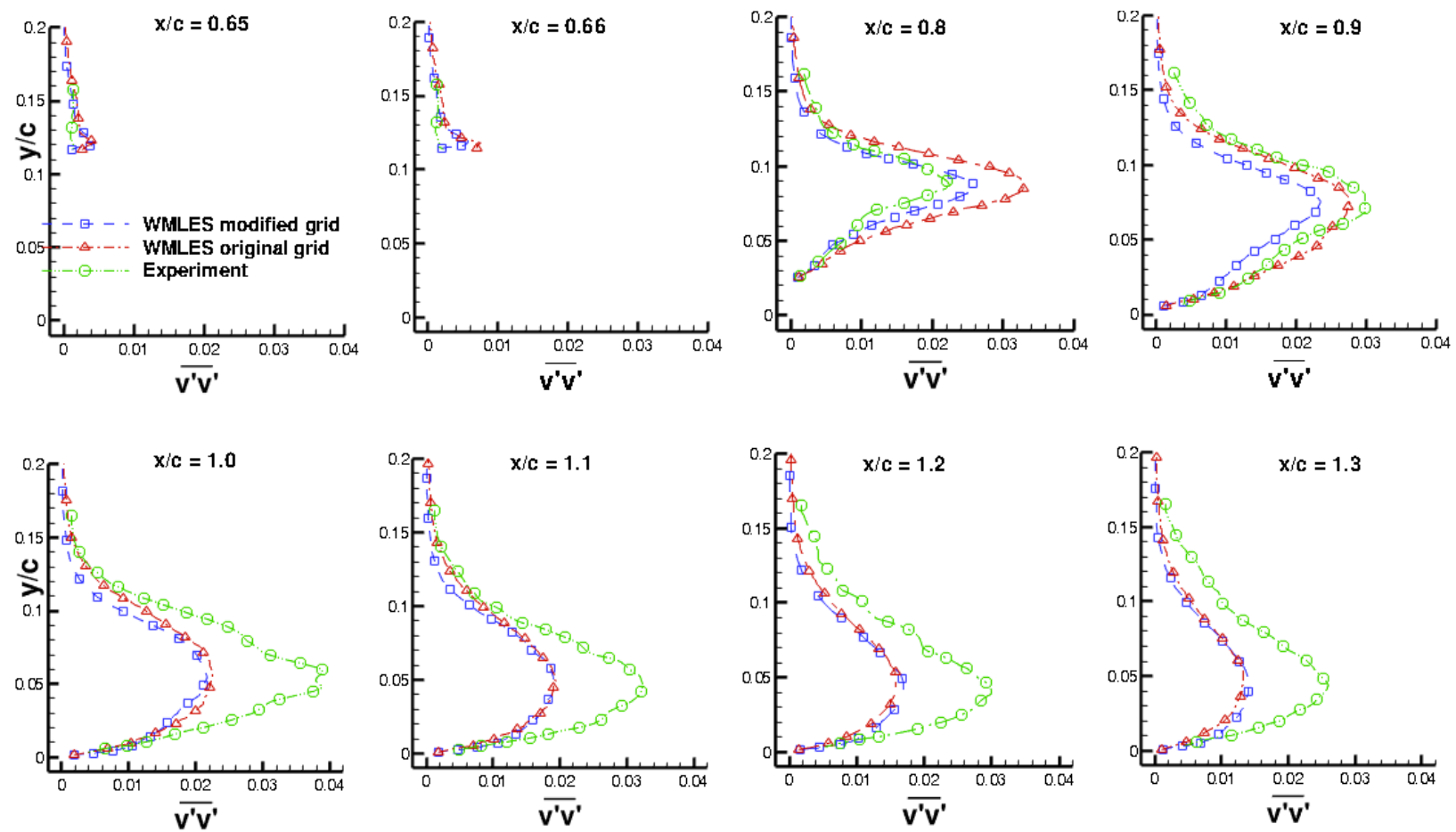

Figure A-9: Profile of $\overline{v^{\prime} v^{\prime}}$
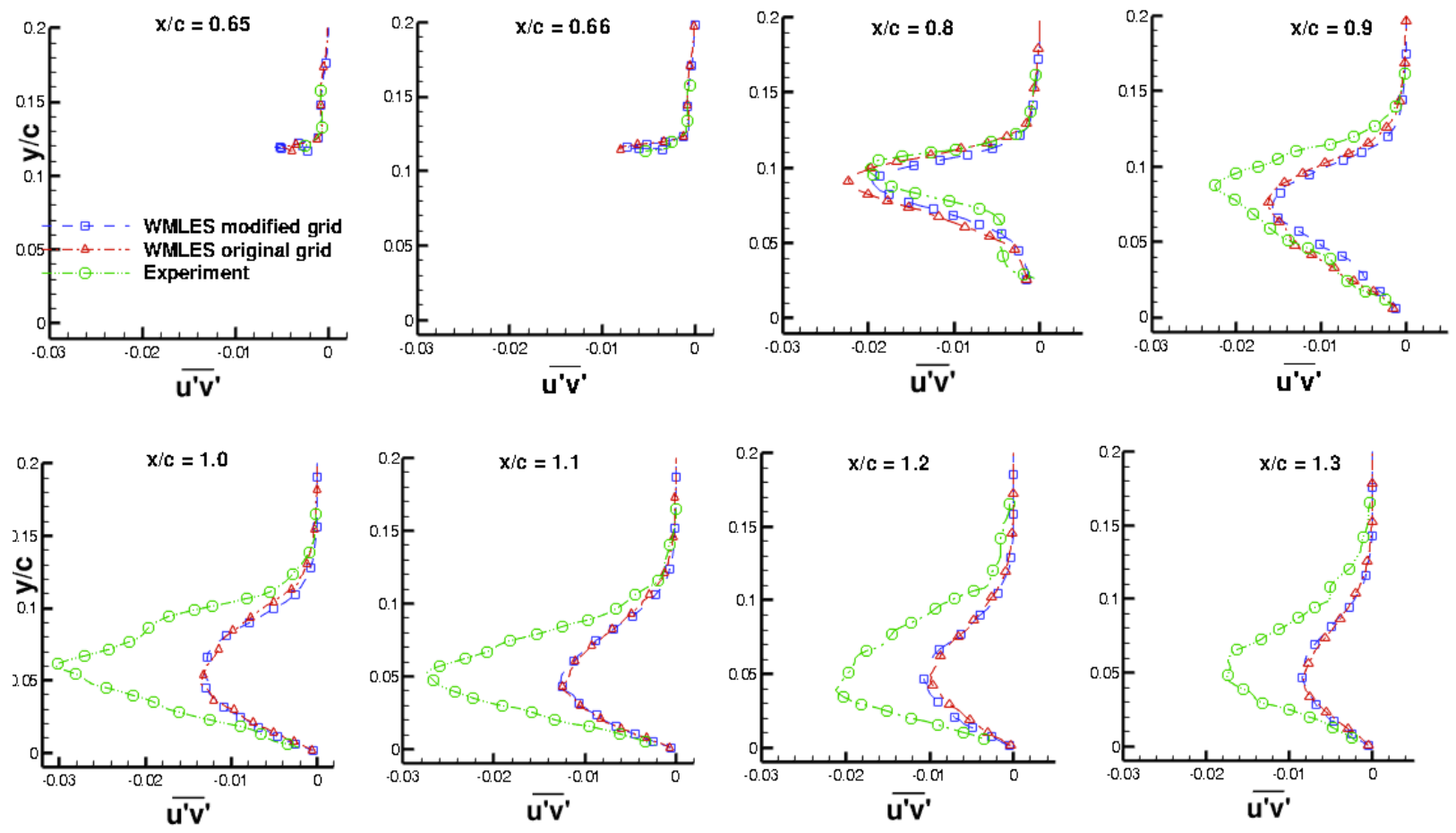

Figure A-10: Profile of $\overline{u^{\prime} v^{\prime}}$ 

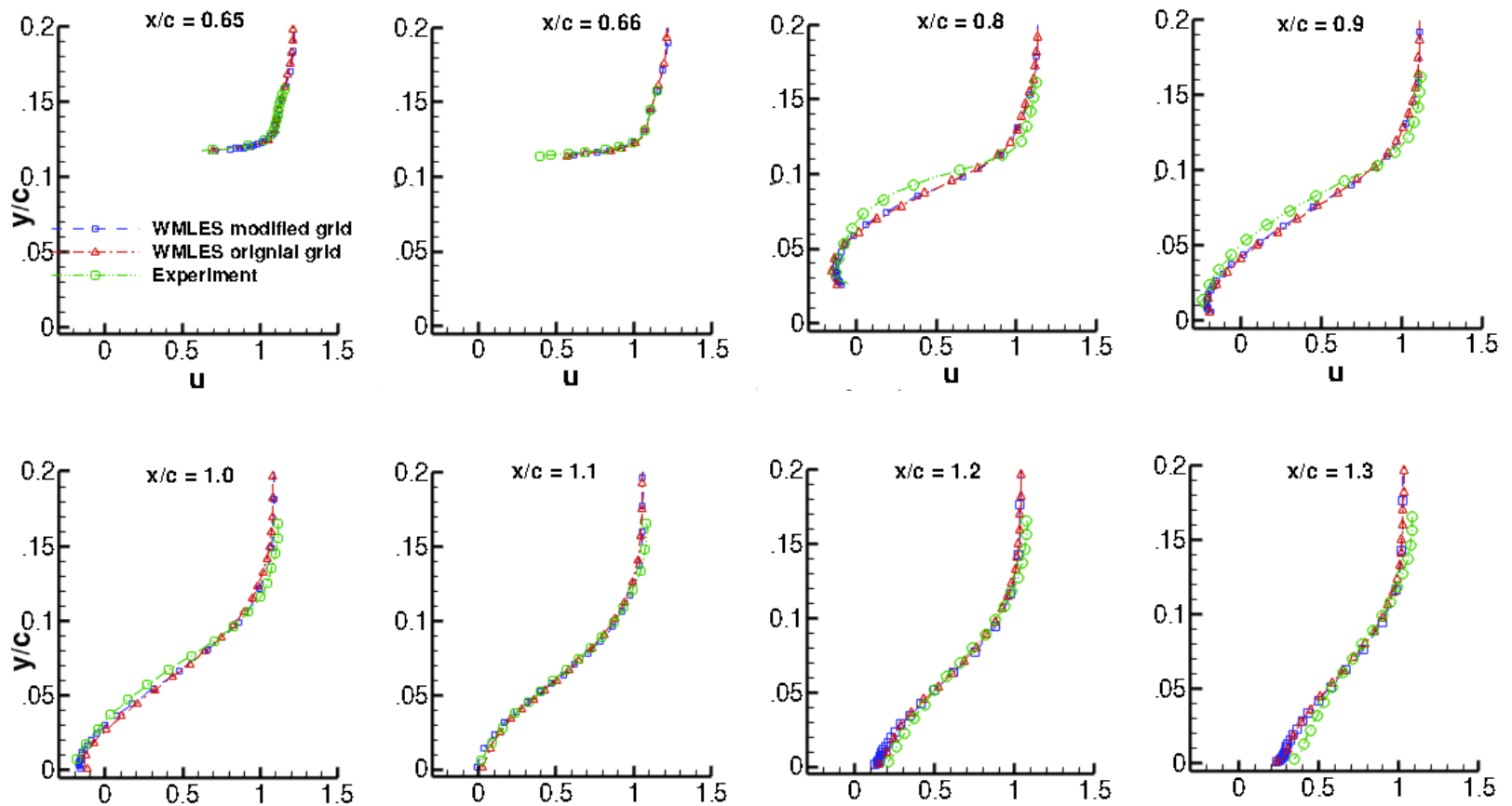

Figure A-11: Profile of streamwise mean velocity

It appears that the change in grid resolution has negligible impact on the profiles of mean velocities and for the most part, the Reynolds stresses as well. With the exception of location 0.8 and 0.9 in case of $\overline{v^{\prime} v^{\prime}}$ and 1.0 and 1.1 in case of $\overline{u^{\prime} u^{\prime}}$ each profile seems to be superimposed on the other. It appears from the plots above that the profile of $\overline{v^{\prime} v^{\prime}}$ move towards the experimental profile in the region beyond the reattachment location $(\sim \mathrm{x} / \mathrm{c}=1.0)$. However, in case of $\overline{u^{\prime} u^{\prime}}$ and $\overline{u^{\prime} v^{\prime}}$ the improvement is only marginal. All the Reynolds stress profiles at location $\mathrm{x} / \mathrm{c}=0.8$ show some improvement, while they continue to exhibit discrepancies with the experiment at other locations. In case of mean velocity profiles, the results from the refined grid shows better agreement with the experiment up till the reattachment point, beyond which discrepancies still exist. 